\title{
Monitoring the Propagation of Lung Sounds Using Electronic Stethoscope Arrays
}

\author{
Submitted by: \\ Kyle R. Mulligan, B. Eng \\ A thesis submitted to the Faculty of Graduate Studies and Research in partial fulfillment \\ of the requirements for the degree of \\ Master of Applied Science \\ Ottawa-Carleton Institute for Biomedical Engineering \\ Department of Systems and Computer Engineering \\ Carleton University \\ Ottawa Ontario, K1S 5B6 \\ Canada \\ September 2009
}

CCopyright Kyle R. Mulligan, 2008 
Library and Archives Canada

Published Heritage

Branch

395 Wellington Street Ottawa ON K1A ON4 Canada
Bibliotheque et

Archives Canada

Direction du

Patrimoine de l'édition

395 , rue Wellington

Ottawa ON K1A ON4

Canada
Your file Votre reférence
ISBN: 978-0-494-60221-8
Our file Notre reference
ISBN: $978-0-494-60221-8$
NOTICE:

The author has granted a nonexclusive license allowing Library and Archives Canada to reproduce, publish, archive, preserve, conserve, communicate to the public by telecommunication or on the Internet, loan, distribute and sell theses worldwide, for commercial or noncommercial purposes, in microform, paper, electronic and/or any other formats.

The author retains copyright ownership and moral rights in this thesis. Neither the thesis nor substantial extracts from it may be printed or otherwise reproduced without the author's permission.
AVIS:

L'auteur a accordé une licence non exclusive permettant à la Bibliothèque et Archives Canada de reproduire, publier, archiver, sauvegarder, conserver, transmettre au public par télécommunication ou par l'Internet, prêter, distribuer et vendre des thèses partout dans le monde, à des fins commerciales ou autres, sur support microforme, papier, électronique et/ou autres formats.

L'auteur conserve la propriété du droit d'auteur et des droits moraux qui protège cette thèse. Ni la thèse ni des extraits substantiels de celle-ci ne doivent être imprimés ou autrement reproduits sans son autorisation.
In compliance with the Canadian Privacy Act some supporting forms may have been removed from this thesis.

While these forms may be included in the document page count, their removal does not represent any loss of content from the thesis.
Conformément à la loi canadienne sur la protection de la vie privée, quelques formulaires secondaires ont été enlevés de cette thèse.

Bien que ces formulaires aient inclus dans la pagination, il n'y aura aucun contenu manquant. 


\section{Abstract}

This thesis presents the design and prototype testing of a novel medical instrument designed to measure changes in to acoustic transmission properties of lung tissue. Since tissue acoustic transmission is largely determined by the distribution of lung fluid and lung tissue density, this instrument has potential applications for monitoring and diagnosis of patients with such obstructive lung diseases which are associated with accumulation of lung fluid and collapse of lung tissue. The apparatus consists of an array of 4 electronic stethoscopes linked together via a fully adjustable harness. A White Gaussian Noise (WGN) input sound is injected into the mouth via a modified speaker and measured on the surface of the chest using the array of stethoscopes. Data were analysed using the Normalized Least Mean Squares (NLMS) adaptive filtering algorithm to develop a transfer function based on the propagation characteristics of the injected signal. This transfer function is then analysed to determine the frequency response and the propagation delay at each stethoscope. The system was calibrated to account for delays in the signal acquisition equipment and verified using a chest phantom model. System non-linearites were analysed and determined to be sufficiently small to justify the linear model. Phantom test results show that as the volume of fluid in the lungs increases, the sound propagation delay decreases. In-vivo results were measured on healthy volunteers and show comparable results to the lung phantom with no volume of water injected and that the instrument can detect sound propagation delay variations with changes in posture. Based on these results, this instrument is able to measure parameters of the lungs including propagation delay and frequency/impulse responses, which show useful correspondence to known physiological changes. 


\section{Acknowledgements}

I would like to thank the following people for their support and encouragement in this project: Dr. Andy Adler, Dr. Rafik Goubran, Michael Mulligan, Gloria Mulligan, and Kirk Mulligan. Without these individuals, I would not have had the inspiration or the determination to complete a successful Master's Thesis. I would also like to acknowledge the Natural Sciences and Engineering Research Council (NSERC) for partially funding this project. 


\section{Table of Contents}

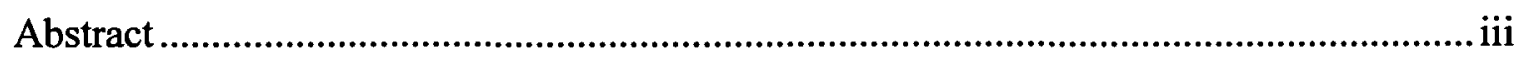

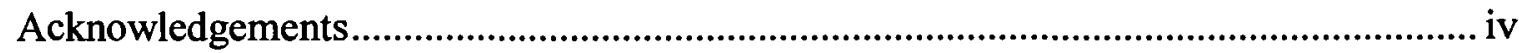

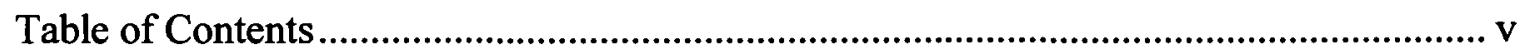

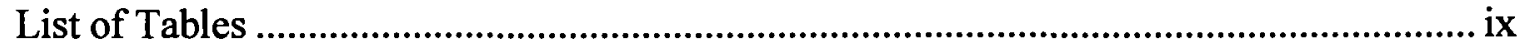

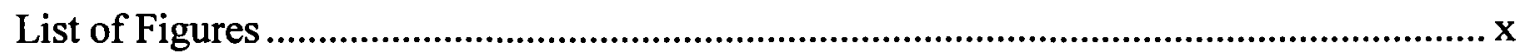

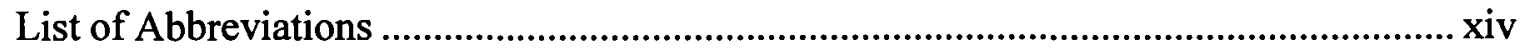

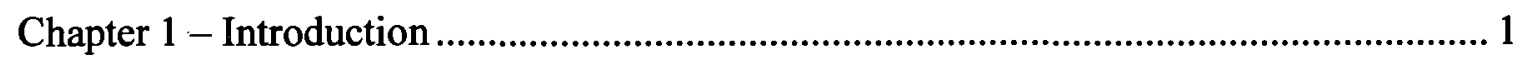

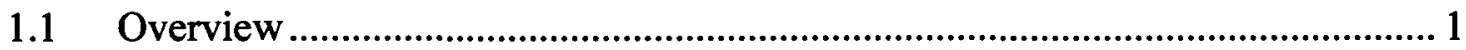

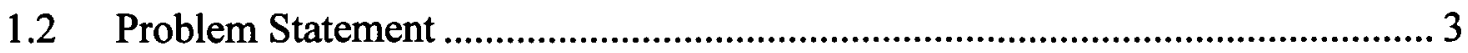

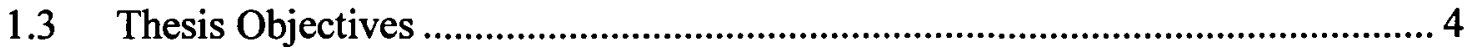

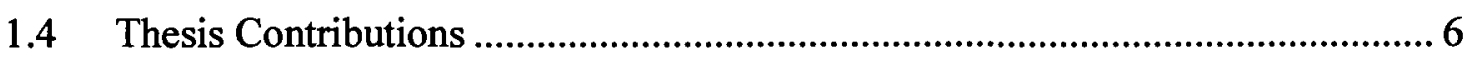

1.5 Thesis Organization........................................................................................ 8

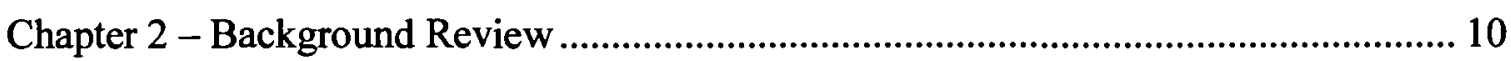

2.1 Overview of Human Lung Anatomy .................................................................... 10

2.1.1 Obstructive Lung Diseases ......................................................................... 12

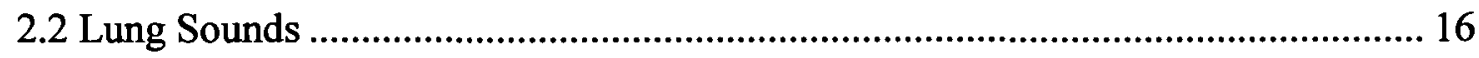

2.2.1 Acoustical Properties of the Lungs and Thorax............................................. 17

2.3 The Stethoscope ............................................................................................... 18

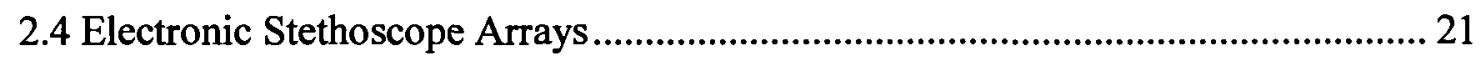

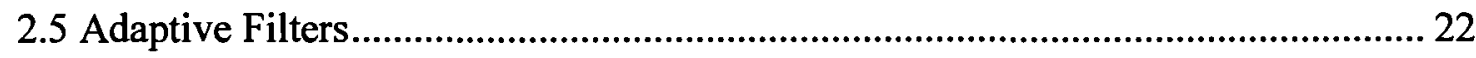

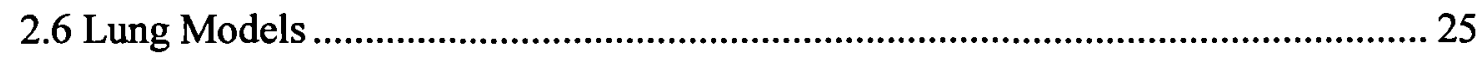




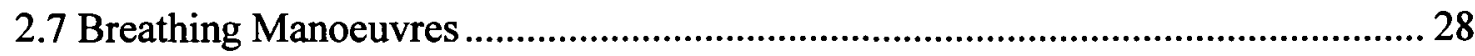

2.7.1 Pursed Lip Breathing Technique ............................................................... 28

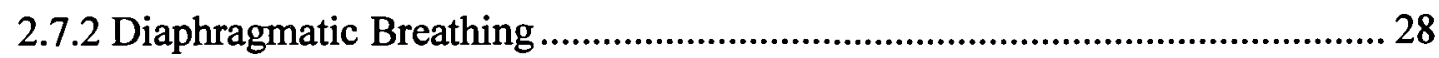

2.8 Current Lung Monitoring Technologies ................................................................ 29

2.8.1 Positron Emission Tomography (PET) .......................................................... 29

2.8.2 Magnetic Resonance Imaging (MRI) .......................................................... 30

2.8.3 Electrical Impedance Tomography (EIT) ……............................................ 30

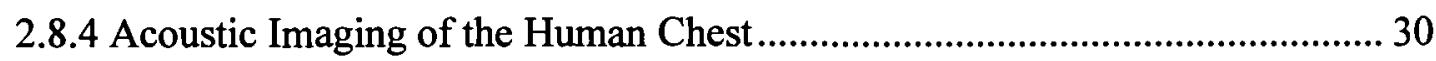

Chapter 3 - Measurement Apparatus Setup....................................................................... 33

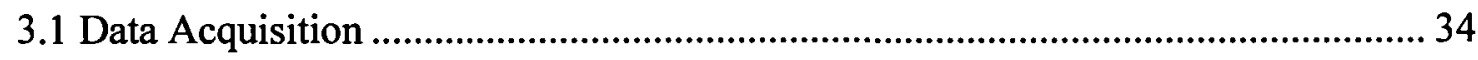

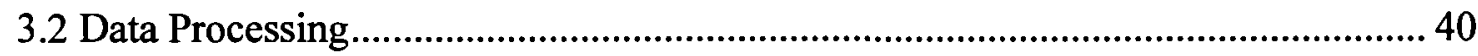

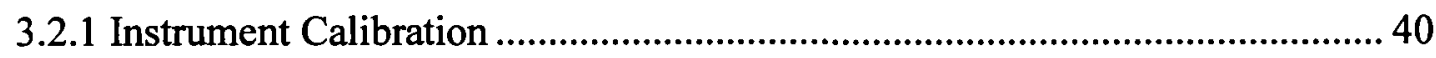

3.2.2 Algorithm Implementation.............................................................................. 46

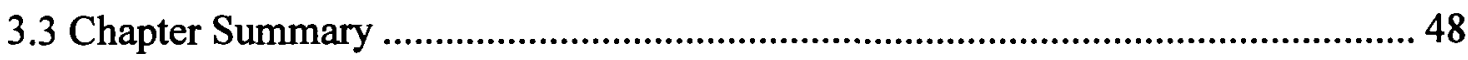

Chapter 4 - Simulation Results........................................................................................ 48

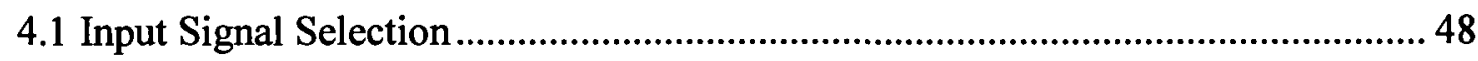

4.2 Adaptive Filter Parameter Selection ..................................................................... 49

4.3 Verification with Adaptive Filter Simulator and FFT ............................................50

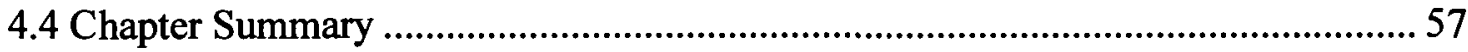

Chapter 5 - In Vitro Chest Phantom Experiments........................................................... 58

5.1 Open Air Column Phantom .................................................................................. 58

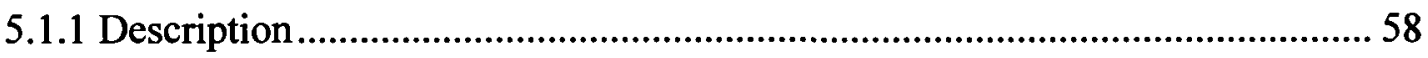

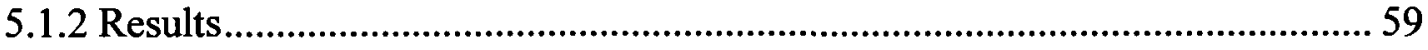




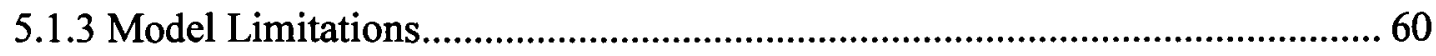

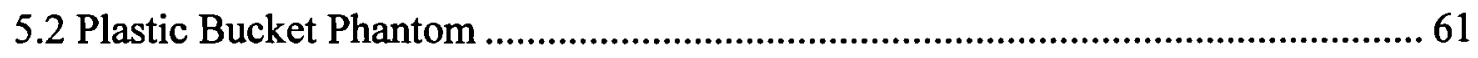

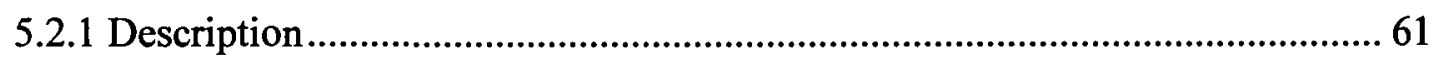

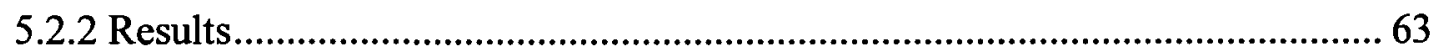

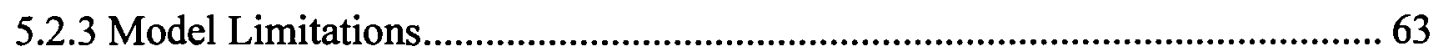

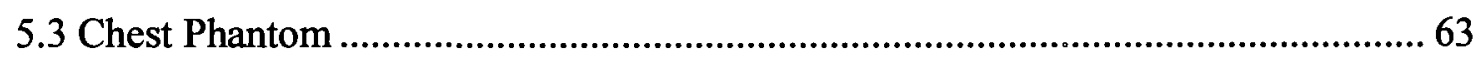

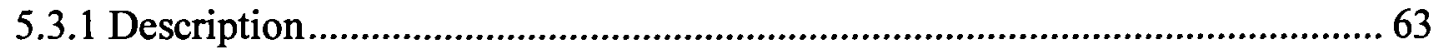

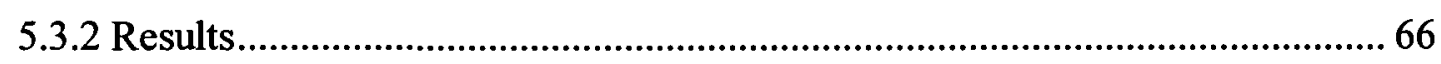

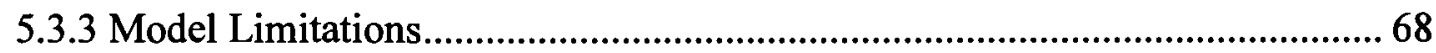

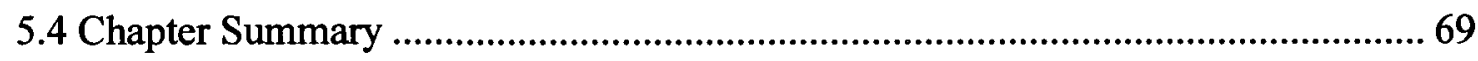

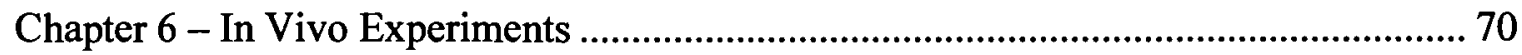

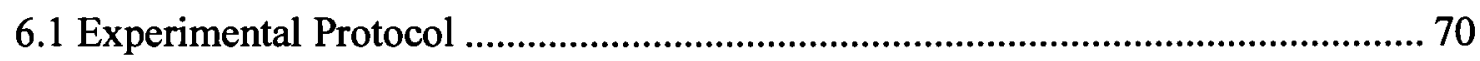

6.2 In Vivo Results - Patient Sitting.......................................................................... 71

6.3 In Vivo Results - Patient Lying on Back................................................................... 72

6.4 In Vivo Results - Lying on Right Side ................................................................... 73

6.5 In Vivo Results - Lying on Left Side .................................................................. 76

6.6 In Vivo Results - Flat on Stomach ....................................................................... 78

6.7 In Vivo Results - All Stethoscopes .................................................................... 79

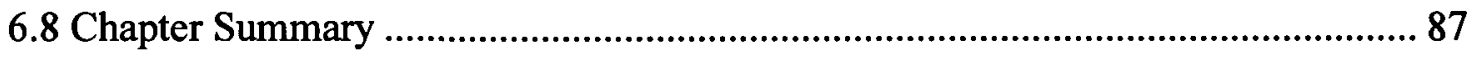

Chapter 7 - Non-Linearities within the System............................................................... 84

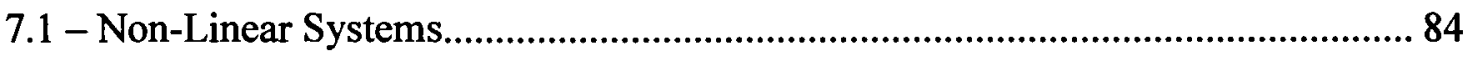

7.2 - Non-Linearities within the System Apparatus ...................................................... 84

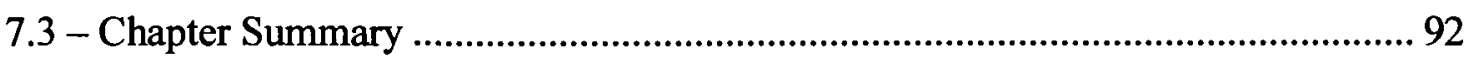


Chapter 8 - Conclusions and Future Work ................................................................... 93

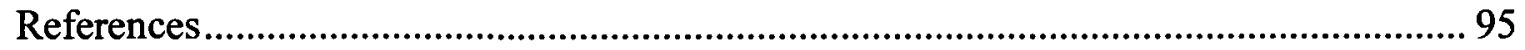




\section{List of Tables}

Table 1 - Acoustical Impedance for Various Organs within the Thorax (Lundqvist, 2008)...17

Table 2 - Features of the Electronic Stethoscope (DS32A Digital Electronic Stethoscope) ...21

Table 3 - Materials used to Construct the Stethoscope Array Harness.......................................36

Table 4 - Components used to construct the sound emitting and recording apparatus.............39

Table 5 - Experimental Results that Compare Sound Propagation Delay Calculations

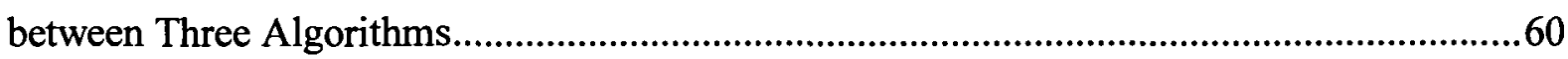

Table 6 - Materials used to build the Plastic Bucket Model .......................................................61 


\section{List of Figures}

Figure 1 - The Human Respiratory System (Reproduced from Illustration of Human

Respiratory System, 2008)

Figure 2 - X-Rays demonstrating a patient with healthy lungs (A) and a patient diagnosed with pneumonia (B). An accumulation of mucus (shown in white) in the patient's right lung can be observed...................................................................................................... 15

Figure 3 - Anterior and Posterior View of the Human Thorax with Auscultation Points (17) (Reproduced from Lung Sound Auscultation Trainer, 2008)...................................... 16

Figure 4 - Mechanical Stethoscope Frequency Response (Reproduced from Webster, 1998) 20

Figure 7 - Example Lung Model (Reproduced from (McKee, 2004)) ............................ 26

Figure 8 - Example Lung Model (Reproduced from (McKee, 2004)) ….......................... 27

Figure 9 - Sketch of Measurement Apparatus and Setup on a Patient................................ 34

Figure 10 - Fully Adjustable Stethoscope Array Harness Attached to a Human Participant

Figure 12 - Actual Off the Shelf Components of the Medical Instrument 39

Figure 13 - Medical Instrument Block Diagram of Off the Shelf Components with Internal Components

Figure 14 - Face Panel of Firepod Preamplifier. Boxes Show Channels 1 and 2 of the Preamplifier.

Figure 15 - Detecting the Delay of the Pre-Amplifier ........................................................ 42

Figure 16 - NLMS Coefficients upon Convergence of the Filter 
Figure 17 - Detecting Delay of the Speaker.

Figure 18 - NLMS Coefficients Showing the Delay between the Laptop Computer, Pre-

Amplifer, and the Speaker Amplifier. .45

Figure 19 - Detecting Delay of the Speaker Transducer, Funnel, and Stethoscope......... 45

Figure 20 - NLMS Coefficients showing the Delay between the Speaker Transducer,

Funnel, and Stethoscope 46

Figure 21 - Data Processing System Setup. 47

Figure 22 - Various Frequency Responses of a Chest Phantom to Determine the Appropriate Input Signal Frequency Spread. 49

Figure 23 - Adaptive Filter Simulator Developed by the Department of Systems and

Computer Engineering Carleton University. 51

Figure 24 - Comparison of Adaptive Filtering Program Model Result with Adaptive

Filtering Simulator Model Result. 53

Figure 25 - Comparison of Instruments Algorithm Model Result with Adaptive Filtering Simulator Model Result over 50 Trials. .54

Figure 26 - Comparison of Instruments Algorithm Model Result with FFT Model Result. 55

Figure 27 - Comparison of Instruments Algorithm Model Result with FFT Model Result over 50 Trials. 56

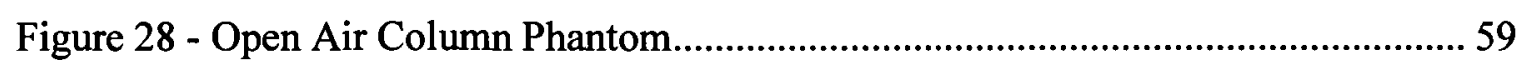

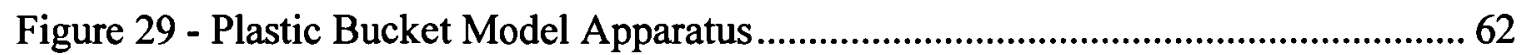

Figure 30 - Chest Phantom (Unknown System) with the Stethoscope Array and Harness Attached to the Tire Inner Tube or Simulated Chest Surface 64 
Figure 31 - Illustration of the Stethoscope Array and Inner Tire Tube Assembly. Water Injected into the Tube Flows to the Bottom due to Gravity. 65

Figure 32 - Propagation Delay for a Stethoscope Without the Presence of Water Within its Field of View. 66

Figure 33 - Propagation Delay for a Stethoscope with the Presence of Water Within its Field of View 67

Figure 34 - Above is the magnitude response of the Transfer Function of the Chest Phantom Model with No Volume, $20 \mathrm{cc}$, and $40 \mathrm{cc}$ of Water. 68

Figure 35 - Transfer Functions for 3 Healthy Human Chests (Sitting Upright)............... 72

Figure 36 - Transfer Functions for 3 Healthy Human Chests (Lying on Back)............... 73

Figure 37 - Transfer Function for 3 Healthy Human Chests of Stethoscope 2 (Channel 3)

Placed over their Left Lung. Participants are Lying on their Right Side. 75

Figure 38 - Transfer Function for 3 Healthy Human Chests of Stethoscope 4 (Channel 5)

Placed over their Right Lung. Participants are Lying on their Right Side. 76

Figure 39 - Transfer Function for 3 Healthy Human Chests of Stethoscope 2 (Channel 3)

Placed over their Left Lung. Participants are Lying on their Left Side. .77

Figure 40 - Transfer Function for 3 Healthy Human Chests of Stethoscope 4 (Channel 5)

Placed over their Left Lung. Participants are Lying on their Left Side. 78

Figure 41 - Transfer Function for 3 Healthy Human Chests. Participants are lying flat on their stomachs. 79

Figure 42 - Frequency Responses of Participant 1 in all Postures Plotted Together........ 80 Figure 43 - Frequency Responses of Participant 2 in all Postures Plotted Together........ 81

Figure 44 - Frequency Responses of Participant 3 in all Postures Plotted Together........ 82 
Figure 45 - Frequency Response for Participant 3 Lying on Back for all Stethoscopes.

The Curve Labelled 1 is the Response on the Stethoscope that was Expected to Yield the

Lowest Propagation Delay. .83

Figure 46 - Frequency Response for Participant 3 Lying on Stomach for all Stethoscopes. The Curve Labelled 1 is the Response on the Stethoscope that was Expected to Yield the Lowest Propagation Delay. 84

Figure 47 - Frequency Response for Participant 3 Lying on the Right Side for all Stethoscopes. The Curve Labelled 1 is the Response on the Stethoscope that was Expected to Yield the Lowest Propagation Delay. 85

Figure 48 - Frequency Response for Participant 3 Lying on the Left Side for all

Stethoscopes. The Curve Labelled 1 is the Response on the Stethoscope that was

Expected to Yield the Lowest Propagation Delay. 86

Figure 49 - Impulse Responses of the System at Varying Volume Levels for the Input

Signal

Figure 50 - Block Diagram of the Modified Lung Monitoring System in or to Detect the Source of Non-Linearities. 88

Figure 51 - Impulse Response of the Loudspeaker at Varying Input Signal Volumes..... 89 Figure 52 - Normalized Impulse Response of the Loudspeaker at Varying Input Signal Volumes 90

Figure 53 - Zoomed Plot of Normalized Impulse Response of the Loudspeaker at Varying Input Signal Volumes. Second Peak at Volume Levels of $20 \%$ and $40 \%$ Match Closely compared to Other Volume Levels 


\section{List of Abbreviations}

$\begin{array}{ll}\text { COPD } & \text { Chronic Obstructive Pulmonary Disease } \\ \text { ECG } & \text { Electro Cardiogram } \\ \text { EIT } & \text { Electrical Impedance Tomography } \\ \text { FFT } & \text { Fast Fourier Transform } \\ \text { GE } & \text { General Electric } \\ \text { HP } & \text { Hewlett Packard } \\ \text { MRI } & \text { Magnetic Resonance Imaging } \\ \text { NLMS } & \text { Normalized Least Mean Squares } \\ \text { OTS } & \text { Off the Shelf } \\ \text { PET } & \text { Positron Emission Tomography } \\ \text { TI } & \text { Texas Instruments } \\ \text { VILI } & \text { Ventilator Induced Lung Industry } \\ \text { VRI } & \text { Vibration Response Imaging } \\ \text { WGN } & \text { White Gaussian Noise }\end{array}$




\section{Chapter 1 - Introduction}

\subsection{Overview}

Auscultation is a technique used to measure sounds within the body (Bergstresser, Ofengeim, Vyshedskiy, Shane, \& Murphy, 2002). Before the invention of the stethoscope in 1819 by Laennec, physicians performed auscultation with the placement of their ears directly on patient's chests or abdomens (Auscultation, 2008). From 1819 to the present, sounds within the body continue to be measured using a stethoscope to examine the cardiovascular (Martinez-Alajarin, Lopez-Candel, \& Ruiz-Merino, 2007), respiratory (Leuppi, et al., 2005), and gastrointestinal systems (Bray, Reilly, Haskin, \& McCormack, 1997). Traditionally, stethoscopes have been used as a preliminary assessment instrument (Kaelin, 2001) to screen patients for disease, after which patients may be referred for more accurate methods such as spirometry, X-Rays, blood pressure cuffs, etc. have that been used as confirmation techniques (Pneumonia) (Webster, 1998).

When using stethoscopes, patients may be misdiagnosed with a disease as a result of the variability in a physician's auditory training. Furthermore, the technique of applying the stethoscope to the patient and breath variability between measurement points can greatly affect the sounds perceived by the physician (Webster, 1998). For example, listening to breathe sounds through a patient's gown or clothing or allowing the tubing of the stethoscopes to rub against the bed rails can cause adventitious sounds that superimpose on a patient's breath sounds, which may be misinterpreted to indicate the existence of 
phantom diseases (Kaelin, 2001). Many errors in stethoscope auscultation can be avoided depending on the physician's training and experience.

Stethoscope auscultation involves the physician listening to many auscultation points on the chest and abdominal areas of a patient's body. At each of the auscultation points, patients are asked to inhale and then exhale. Patients may seek positive reinforcement from the physician meaning that they may constantly try to overcome their disease by forcing themselves to breath normally in order to receive acceptance from the physician (Carlson, Buskist, Enzle, \& Heth, 1997). This may prevent the physician from accurately detecting any diseases within the patient.

Variability in stethoscope auscultation can be reduced between breath sounds at each of the auscultation points using an array of electronic stethoscopes and computers due to the enablement of simultaneous sound capture at each of the auscultation points, thus requiring only one patient breath. Secondly, with the use of electronic stethoscopes, sounds captured from each of the individual stethoscopes can be captured and saved using a computer. These sounds can then be played back to the physician individually, simultaneously, or in many other ways using a variety of techniques (McKee, 2004).

Finally, another benefit with using electronic stethoscopes and computers is that signalprocessing algorithms can be developed to provide computer-aided diagnostic system tools in order to help eliminate variability and uncertainty between the diagnoses between physicians. 


\subsection{Problem Statement}

This thesis aims at investigating the analysis of sound propagation though the human chest using adaptive filtering techniques and the electronic stethoscope in order to develop a computer-aided medical instrument capable of measuring changes in the distribution and density of fluid within the lungs caused by diseases such as asthma, bronchitis, emphysema, and pneumonia. Possible medical applications for the instrument include the determination of treatment effectiveness for fluid related respiratory diseases or in the development of ventilation strategies to avoid Ventilator Induced Lung Injury (VILI) through analysis of relative volume changes of mucus in the anterior and posterior chest and abdominal regions following the initiation of a treatment or changes in patient posture. The instrument is also capable of providing spatial information to determine the location of an obstruction or build up of mucus within the pulmonary system.

Electronic stethoscope arrays are used to eliminate breath variability between auscultation points such that one breath is captured at all of the auscultation points instead of measuring many breaths at each auscultation point with one stethoscope providing a means to more accurately diagnose and monitor lung diseases. The electronic stethoscope array allows sounds captured from the chest to be read in and stored by a computer on its hardrive such that they may be loaded and analyzed using any audio software package. A custom audio software tool could be developed using the data captured by the electronic stethoscope array on the surface of the chest in order to 
provide physicians with the ability to playback the captured sounds in a variety of manners for more accurate diagnoses of pulmonary disorders.

\subsection{Thesis Objectives}

The purpose of this thesis was to study the spatial distribution and density of fluids in the lungs leading to airway blockages which impede breathing. Such fluid accumulation is associated with diseases such as asthma, bronchitis, emphysema, and pneumonia. In order to accomplish this objective, a medical instrument was developed that injects a sound into a patient's mouth while measuring it on the chest surface using an array of electronic stethoscopes placed at each auscultation point on the anterior and posterior chest surfaces. The sound signals that were captured by the instrument were analyzed using a data processing algorithm that implements the Normalized Least Mean Squares (NLMS) adaptive filtering technique. Adaptive filtering is a digital signal processing technique that uses a linear Finite Impulse Response (FIR) filter with adjustable coefficients and a coefficient update algorithm (NLMS) to represent the behaviour of an unknown system.

The software developed for the instrument that implemented the data processing algorithm had many objectives. The first objective was to provide physicians with the ability to playback sounds captured from the chest individually, simultaneously, or in various combinations. Secondly, if the physician determined a suitable treatment for a patient with a respiratory disease, the instrument would provide the physician with an approximate location of respiratory obstructions and a relative change in volume after the 
treatment was administered. The effectiveness of the treatment would be evident if large changes in volume resulted or if the obstruction moved to another location. If none of these events occurred, perhaps another treatment would be more suitable for the disease. Mechanical ventilation is used for patients with respiratory failure caused by failure to ventilate, characterized by increased arterial carbon dioxide tension, or failure to oxygenate, characterized by decreased arterial oxygen tension. The treatment for respiratory failure is to increase the patient's alveolar ventilation (the rate and depth of breathing), either by reversing the cause or by using mechanical ventilation. It has been discovered that lung protective ventilation strategies can be used to drastically improve patient health and reduce injuries to the respiratory system from mechanical ventilation (Dreyfuss \& Saumon, 1998). To protect against Ventilator Induced Lung Injury (VILI), many novel modes of ventilation have been developed; however clinicians have difficulty choosing optimal ventilation parameters, because patients' lungs are highly heterogeneous and change rapidly. The instruments currently available either don't provide regional information (i.e. SpO2), or temporal information (i.e. X-ray CT) (Dreyfuss \& Saumon, 1998). Another objective for the new lung parameters that are obtained in the instruments data processing algorithm including changes in sound propagation delay in the presence of lung fluid, the impulse/frequency response of the chest that yields peak frequencies and under curve area changes, could be used to help improve in the selection of optimal ventilation parameters in order to reduce VILI.

In order to validate the functionality of the medical instrument and avoid excessive use of human participation duration testing phases, a series of homogenous phantom models 
were also developed. The first phantom model was a very simple and well understood model developed for verification of the adaptive filtering data processing algorithm of the medical instrument. Complexities were added to successive models in an attempt to simulate the behaviour of actual human chests to avoid excessive human participation. Upon completion of a reliable computer-aided medical instrument, human trials were conducted and results were compared to the chest phantom model results.

The main emphasis of this thesis was to determine the validity of adaptive filtering as a means to develop a computer-aided medical instrument for determining spatial and density information of the human chest in the presence of respiratory disease. The overall goal of the computer-aided medical instrument was to capture respiratory sounds from four auscultation points around the chest simultaneously and provide a physician with spatial and density information to allow assessment of the effectiveness of the treatment provided to the patient or aid in the development of ventilation strategies in order to reduce VILI.

\subsection{Thesis Contributions}

The following are the major contributions demonstrated in this thesis:

1. Regional lung properties using the audio transfer function of the respiratory system were characterized. Results show that two frequencies in the frequency response of the chest consistently passed through in the range of under $200 \mathrm{~Hz}$. These frequencies were shown as distinct peaks in the frequency response of the human and chest phantom models. Furthermore, if the volume of fluid in the chest 
phantom increased, these frequency peaks became sharper. These results were published in a paper entitled "Detecting Regional Lung Properties using the Audio Transfer Function of the Respiratory System" accepted in the International Conference of the IEEE Engineering in Medicine and Biology Society held in Minneapolis, MN in September 2009 (Mulligan, Adler, \& Goubran, Detecting Regional Lung Properties using the Audio Transfer Function of the Respiratory System, 2009).

2. A medical lung monitoring instrument was developed capable of detecting changes in sound propagation delay as a volume of fluid within an object. The objects consisted of three chest phantom models used to simulate the behaviour of human chests. These results were published in a paper entitled "Monitoring Lung Disease using Electronic Stethoscope Arrays" that was published in the proceedings of the Canadian Medical and Biological Engineering Society Conference held in Calgary, AB in May 2009 (Mulligan, Adler, \& Goubran, 2009).

3. Three chest phantom models were incrementally developed to both verify algorithm implementations of adaptive filtering and to simulate human chest behaviour to sound signals. The final chest model that was developed was used to test the instruments ability to detect changes in sound propagation delay with changes of water volumes within the system. The instruments' ability to localize the excess volume of water was also tested. These results were published in a 
paper titled "Monitoring Lung Disease using Electronic Stethoscope Arrays" published in the proceedings of the Canadian Medical and Biological Engineering Society Conference held in Calgary, AB in May 2009 (Mulligan, Adler, \& Goubran, 2009).

4. Although the modeling algorithm assumed that sound propagation through the chest both phantom and human to be linear, possibilities for nonlinearities were investigated. Nonlinearities were detected from the sound emitting portion of the instrument but could be eliminated by reducing the volume below $80 \%$ of its maximum emitting power.

\subsection{Thesis Organization}

Chapter 1 introduces the problem to be solved and discusses the objectives of this thesis with a light discussion of target respiratory diseases and a medical instrument developed to monitor these diseases.

Chapter 2 provides background information on lung anatomy, stethoscopes, microphone arrays, adaptive filters, breathing manoeuvres and lung models.

Chapter 3 describes the development of an instrument to emit, acquire, and process sound data. 
Chapter 4 discusses results from simulations used to select an appropriate input signal type and frequency range along with reasonable adaptive filter parameters. The chapter also includes test cases used to verify the adaptive filtering software implementation.

Chapter 5 investigates various in vitro chest phantom experiments in order to model human chest behaviour and perform experiments to verify that the developed instrument can detect new parameters.

Chapter 6 describes instrument tests performed on actual human participants. The results are compared to in vitro experiments.

Chapter 7 discusses the effects of nonlinearities within the system and how they affect the performance of the instrument.

Chapter 8 concludes the thesis and looks into possibilities for future research to improve the instruments performance, determine new parameters relevant to disease detection within the chest, investigation into external sound propagation paths. 


\section{Chapter 2 - Background Review}

This chapter discusses background information on lung anatomy, stethoscopes, electronic stethoscope arrays, adaptive filters, lung models, and current technologies for monitoring of the lungs.

\subsection{Overview of Human Lung Anatomy}

The lungs allow air to enter the body such that $\mathrm{O}_{2}$ can become absorbed into the blood stream, and $\mathrm{CO}_{2}$ can leave it. Air is inspired into the lungs through either the nose or the mouth. As air enters through the nose, it is heated to body temperature, and moistened. Foreign particles to the body such as dirt are trapped in the nasal cavity by a thin layer of mucus and then transported via many blood vessels to the digestive system. The digestive system can more effectively dispose of foreign particles when compared to the delicate lungs. When inhaling air through the mouth, mucus lines the larynx and bronchial tubes discussed below to dispose of foreign particles.

The inhaled air travels from the nasal cavity or the mouth into the throat region known as the pharynx. Air channelled through the pharynx then travels through the human voice box referred to as the larynx. Just above the larynx is the epiglottis which is used to control the flow of food versus the flow of air. When a person is eating or swallowing something, the epiglottis remains closed to channel the non-gases to the digestive system. When a person is not swallowing and therefore breathing, the epiglottis remains open to channel gases to the lungs. 
Upon exiting the larynx, air is channelled into the trachea which is a tube that divides into two bronchi whose ends each connect to a lung. Many medium sized particles that have passed through the cleansing systems in the nasal cavity and the larynx are trapped in the bronchi. Many of the particles trapped by the mucus are constantly being beaten upwards into the pharynx by hair-like structures that line the bronchi such that they are swallowed into the digestive tract (Definition: Cilia, 2007).

The lungs are a pair of spongy organs that occupy the thoracic (chest) cavity. As the bronchi branches from the trachea to each lung, branching continues forming more than one million bronchioles in each lung. Finally, at the end of each bronchiole is a cluster of air sacs (alveoli) used to retain inhaled air for gas transfer into the blood stream. Oxygen is transferred through the thin walls of the alveoli into surrounding capillaries to replenish all energy using sources within the body. At the same time, carbon dioxide released into the blood stream from muscles that have used oxygen is transferred into the alveoli to be exhaled (Solomon, Berg, \& Martin, 1999). Figure 1 illustrates the flow of air in the human body along with the names of the anatomical components involved in the breathing process. 


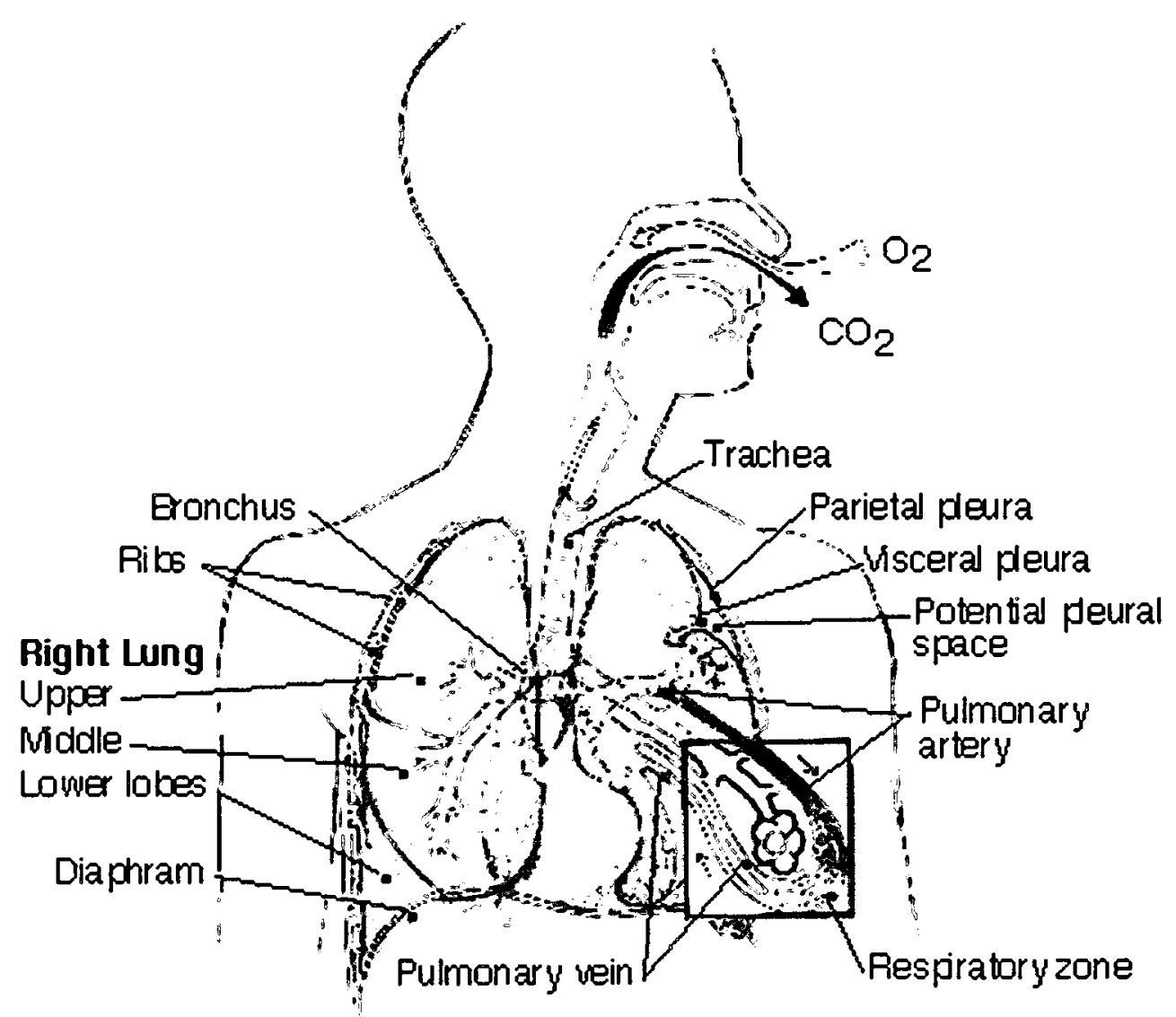

Figure 1 - The Human Respiratory System (Reproduced from Illustration of Human Respiratory System, 2008)

\subsubsection{Obstructive Lung Diseases}

The respiratory system contains several defence mechanisms against foreign particles that may harm the lungs contained in the air that we breathe. Some examples of the defence mechanisms of the respiratory system used to filter air of foreign particles into the digestive system include: hair fibres that line the nostrils, ciliated mucus that line the nose and pharynx, and the cilia-mucus elevator of the trachea and bronchi. The body's most rapid defence mechanism when exposed to foreign particles is bronchial constriction. Bronchial constriction is the narrowing of the bronchial tubes such that there is an increasing chance that foreign particles will collide with the mucus lined walls. The 
bronchus is the final area where the body has defence against foreign particles. Upon reaching the bronchioles or the alveoli, foreign particles are absorbed by cells that are responsible for cleaning cellular debris known as macrophages (Definition: macrophages, 2005). A long term exposure of inhaling foreign particles into the respiratory system can cause lung diseases (Solomon, Berg, \& Martin, 1999).

Lung diseases that cause the body to create an excess of mucus are the main focus of this thesis. Such diseases known are Chronic Obstructive Pulmonary Diseases (COPD) are: asthma, chronic bronchitis, pulmonary emphysema, and pneumonia (Solomon, Berg, \& Martin, 1999). A brief description of each disease will be provided in the following paragraphs.

Asthma is a condition that results from allergies (Marieb, 1995). Bronchial constriction occurs as the respiratory passages swell and fill with an excess of mucus. This increases airway resistance causing victims to wheeze and gasp for air (Maddox \& Schwartz, 2002).

We describe chronic bronchitis as a combination with pulmonary emphysema because often victims of chronic bronchitis develop pulmonary emphysema. Chronic bronchitis is an inflammation of the bronchi (medium-sized airways) in the lungs. Emphysema is an abnormal condition in the lungs sometimes leading to chronic bronchitis because the body reacts to toxic chemicals such as cigarette smoke with production of excess mucus. Both diseases thus lead to symptoms related to victims that have had exposure to an 
excess of air filled with foreign particles. The bronchial tubes react to the pollutants by secreting an excess of mucus in an attempt to trap and dispose the pollutants into the digestive tract. Ciliated cells in the bronchial tubes are damaged by the excess of foreign particles and cannot effectively clear the excess mucus and trapped particles to the digestive tract. The body reacts by coughing to clear the airways. Victims of chronic bronchitis have constricted and inflamed bronchioles and feel short of breath. Over time, the alveoli become less elastic and the tissues between adjacent alveoli deteriorate. This leads to a reduced lung surface area and thus gas exchange and stale air exhalation efficiency are reduced. At this point, the victim of chronic bronchitis has developed pulmonary emphysema and struggles for every breath (Solomon, Berg, \& Martin, 1999).

Pneumonia is an infectious inflammation of the lungs in which mucus accumulates in the alveoli. The foreign particles in the case of pneumonia are infectious bacteria. The body produces an excess of mucus to trap and fight the infectious bacteria. Pneumonia is the sixth most common cause of death in the United States of America (Marieb, 1995). Figure 2 shows two X-Rays of the chest area. The first X-Ray (A) demonstrates a pair of healthy lungs whereas in the second X-Ray (B) an excess of mucus can been seen accumulating in the patient's right lung shown in white. 


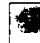

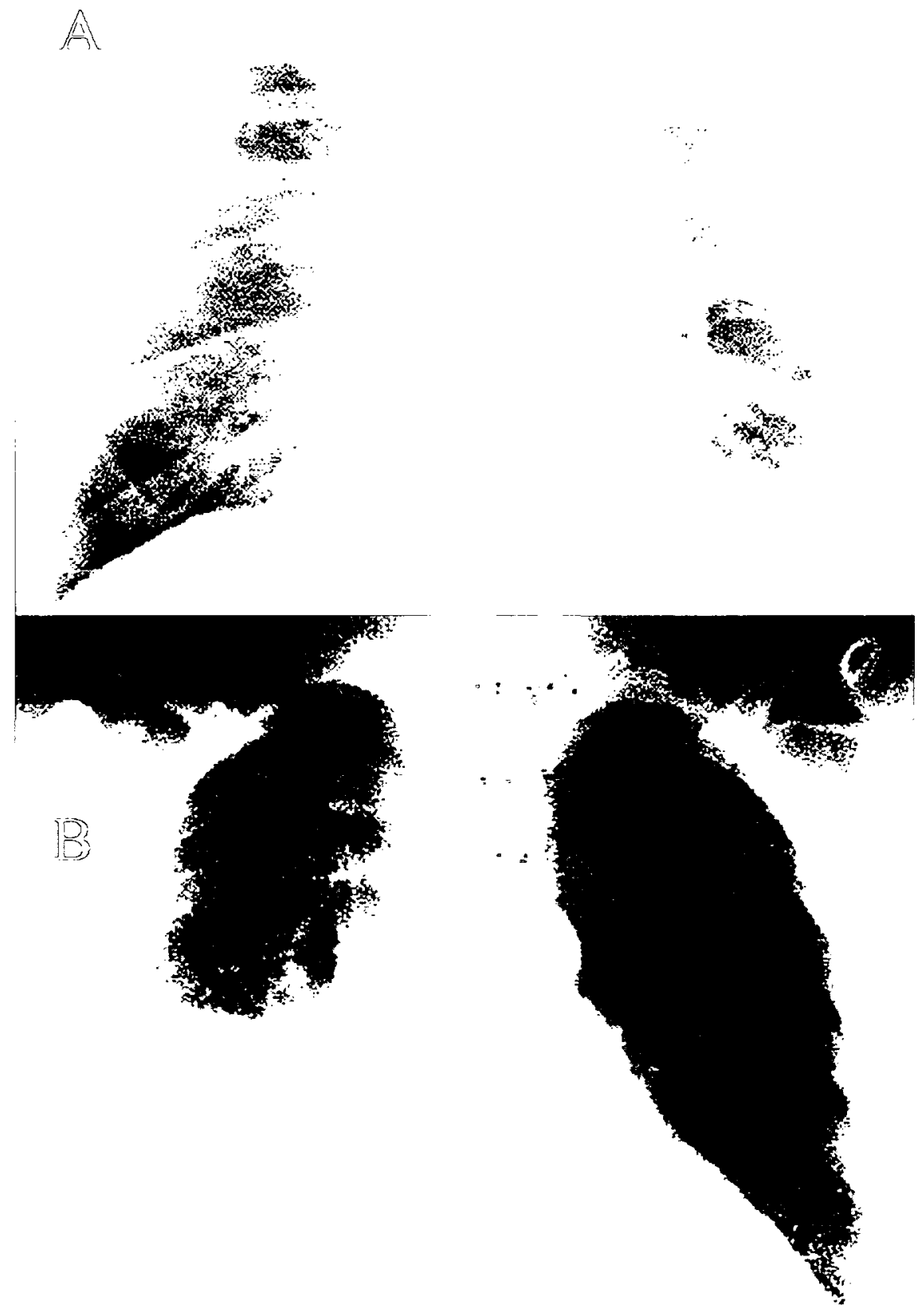

Figure 2 - X-Rays demonstrating a patient with healthy lungs (A) and a patient diagnosed with pneumonia (B). An accumulation of mucus (shown in white) in the patient's right lung can be observed (Reproduced from Computer Tomography (CT), 2009). 


\subsection{Lung Sounds}

There are many sounds produced within the body due to various internal organs such as the heart and lungs. Sounds emitted from the lungs are referred to as breath sounds. Breath sounds are best heard with the use of a stethoscope placed upon various auscultation points of the thoracic area (Schriber, 2007). Figure 3 illustrates the auscultation points where a physician would place a stethoscope to listen to breath sounds in the diagnosis of lung diseases.

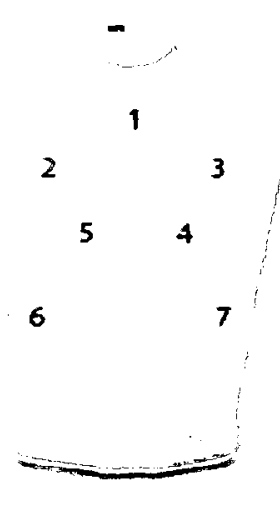
Anterior area
1 trachea
2 upper right lung field
3 upper left lung field
4 middle right lung field
5 middle left lung field
6 lower right lung field
7 lower left lung field

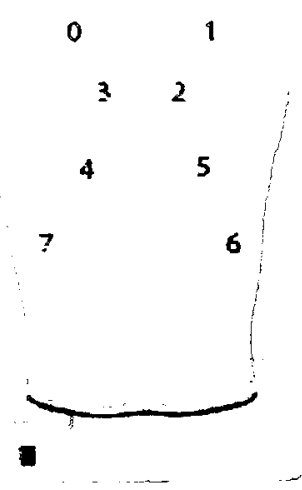

Posterior area

O upper left lung field 1 upper right lung field 2 middle right lung field 3 middle left lung field 4 lower left lung field 5 lower right lung field 6 right costophrenic angle 7 left costophrenic angle

Figure 3 - Anterior and Posterior View of the Human Thorax with Auscultation Points (1-7) (Reproduced from Lung Sound Auscultation Trainer, 2008).

If a patient has contracted a respiratory disease, several types of abnormal breath sounds may occur. Four common types of abnormal breath sounds are: crackles (Rales), rhonchi, stridor, and wheezing (Schriber, 2007). Crackles occur when small airways of the alveoli are collapsed or blocked by mucus. During inhalation if the obstruction is removed, the airway or alveoli "pop" open causing crackles. Similar to crackles, rhonchi are sounds that occur during inhalation due to airways that are lined with an excess of mucus. The mucus causes the airway to resonate producing a sound similar to that of "snoring" 
(Schriber, 2007). Finally stridor, a harsh, grating, or wheezing has been reported as adventitious respiratory sounds in obstructive patients during forced exhalation (Lin, $\mathrm{Wu}$, Chong, \& Chen, 2006).

\subsubsection{Acoustical Properties of the Lungs and Thorax}

The thorax is composed of a variety of organs of varying materials. From the surface of the chest and back to the lung region, there exists skin, fat, bone, blood, and lung tissue each with varying acoustic impedances. Acoustic impedance is the ratio of sound pressure with respect to particle velocity. Table 1 outlines materials found in the thorax with their acoustic impedance.

\begin{tabular}{|c|c|}
\hline Organ & Acoustic Impedance $\left(\mathrm{kg} / \mathrm{m}^{2} / \mathrm{s}\right)$ \\
\hline Skin & $1.63 \times 10^{6}$ \\
\hline Fat & $1.38 \times 10^{6}$ \\
\hline Bone & $7.80 \times 10^{6}$ \\
\hline Lung & $1.63 \times 10^{6}$ \\
\hline Blood & $1.61 \times 10^{6}$ \\
\hline
\end{tabular}

Table 1 - Acoustical Impedance for Various Organs within the Thorax (Lundqvist, 2008)

Many authors have performed measures of sound velocity based on transit time within the thorax by injecting a coded audio signal into the trachea and performing measurements on the chest surface. Work by (Bergstresser, Ofengeim, Vyshedskiy, Shane, \& Murphy, 2002), considered the thorax as a whole to estimate sound velocity in the lungs and therefore did not have to consider the individual organs within the thorax. Sound velocity was calculated to be $37 \mathrm{~m} / \mathrm{s}$ which is not consistent with results from 
(Paciej, Vyshedskiy, Shane, \& Murphy, 2002), determining a sound velocity of $22 \mathrm{~m} / \mathrm{s}$ and (Rice, 1982), determining a sound velocity between 25 and $70 \mathrm{~m} / \mathrm{s}$. All authors agree, however, that sound transit times decrease as lung volume increases.

Others such as (Kompis, Pasterkamp, \& Wodicka, 2001) have used the information of sound velocity in the thorax and microphone arrays to calculate three-dimensional data arrays of sound sources in the thorax and represent the result graphically to construct images of the lungs. Much of their work has been developed using relatively homogeneous phantom models of the thorax using materials such as: gelatine to represent solid tissue and the lung parenchyma, and rubber to represent the trachea and various airways. When the imaging system was completed and tested on humans, results were poor because the human thorax is not homogeneous in nature. It is evident (Table 1) that there are varying acoustic impedances within the thorax and thus sound transit times that are critical for building the three-dimensional data array used in lung imaging must be accounted for.

\subsection{The Stethoscope}

Stethoscopes are used to transmit sounds from the surface of the body to the human ear. There is much variability in interpretation of sounds received from stethoscopes due to auditory acuity and training. Furthermore, depending on the placement of the stethoscope to the desired surface of the body, some sounds may be attenuated or perceived differently. 
The frequency response of the mechanical stethoscope, illustrated in Figure 4, was determined by (Ertel, Lawrence, Brown, \& Stern, 1966) by applying a known audio frequency signal to the end of the stethoscope via a coupling mechanism. It can be seen from Figure 4 that the frequency response of the mechanical stethoscope is uneven and appears to have a low pass filtering effect. Furthermore, critical information exists from the stethoscope near the lower threshold of human hearing. This information can often be missed if a physician does not have perfect hearing in the lower frequency ranges. Therefore, physicians using a mechanical stethoscope may not detect important low frequency sounds. Also, the mechanical stethoscope is only capable of detecting low frequency sounds under around $1000 \mathrm{~Hz}$ from Figure 4. Using electronic stethoscopes will allow measurement of higher frequency sounds to pass through the body and thus anatomical information may be ignored. 


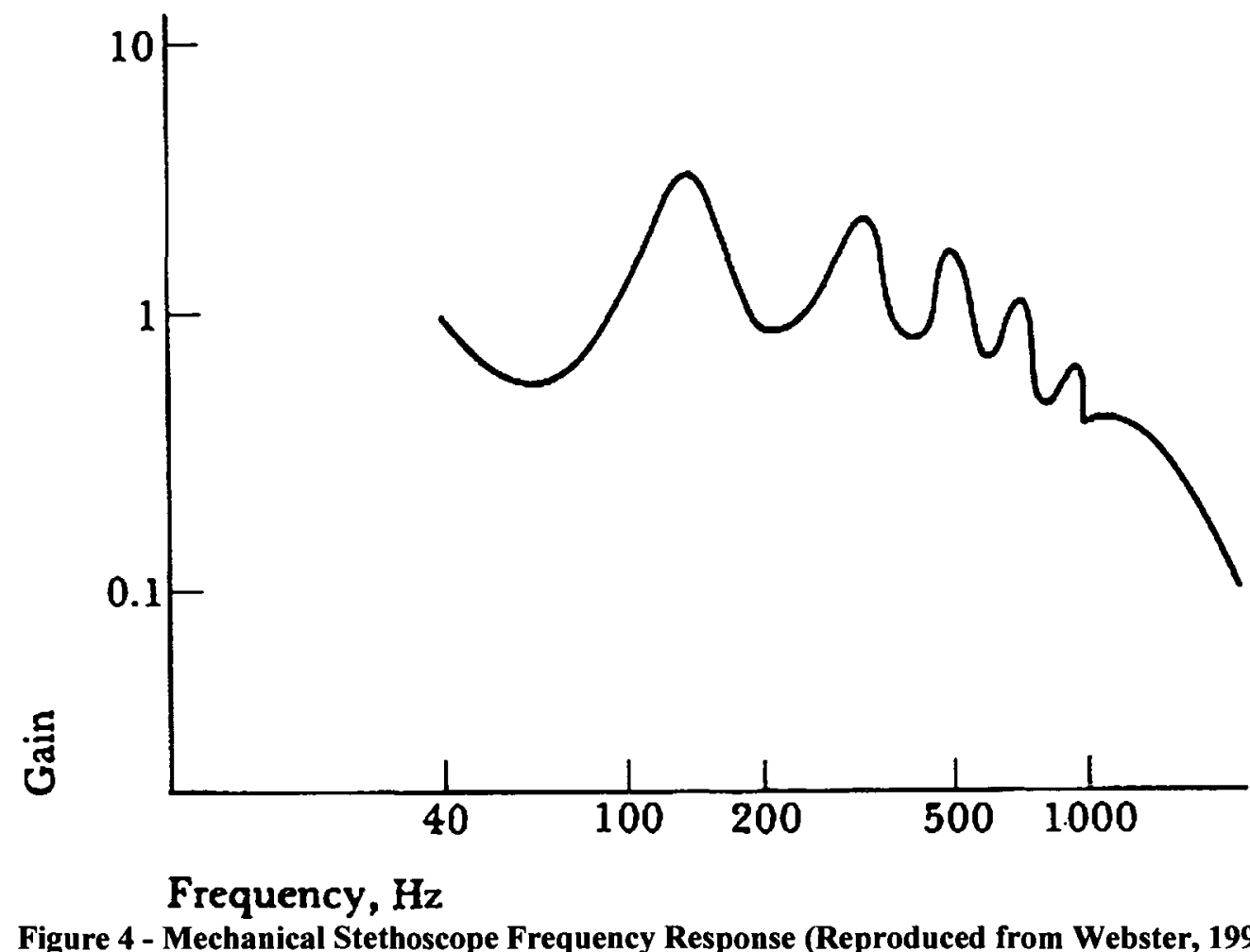

Figure 4 - Mechanical Stethoscope Frequency Response (Reproduced from Webster, 1998)

Also, if the stethoscope malfunctions and attenuates the captured sound signals more than $3 \mathrm{~dB}$, the sound signal will be completely lost to the physician using the stethoscope due to a low signal to noise ratio (SNR) (Webster, 1998). The attenuation of sound varies between stethoscopes and therefore a physician may hear sounds from one stethoscope and not another.

Placement of the stethoscopes chest piece may also affect captured sounds. Placing the chest piece too firmly will severely low-pass filter the received signals. This low-pass filtering effect occurs because the skin forms a diaphragm at the chest piece rim. The diaphragm pressurizes and thus attenuates low frequencies. 
For reasons outlined above, engineers have developed electronic stethoscopes. Modern electronics allows for adjustable frequency responses and therefore more valuable acoustical information may be extracted from the thorax (Webster, 1998). Electronic stethoscopes provide features outlined in Table 2 .

\begin{tabular}{|c|c|}
\hline Feature & Description \\
\hline Amplification & $\begin{array}{l}\text { Amplifies the captured signal up to } 50 \\
\text { times its normal amplitude. }\end{array}$ \\
\hline Acoustic Modes & $\begin{array}{l}\text { Allows the physician the ability to use the } \\
\text { electronic stethoscope as a traditional } \\
\text { stethoscope or use the advanced features of } \\
\text { new technology (varying frequency } \\
\text { responses). }\end{array}$ \\
\hline Ambient Noise Rejection & $\begin{array}{l}\text { Reject background noise for the external } \\
\text { environment that may lead to misdiagnosis. }\end{array}$ \\
\hline Audio Input/Output & $\begin{array}{l}\text { Allows for sound capture into a PC for } \\
\text { sound analysis. }\end{array}$ \\
\hline
\end{tabular}

Table 2 - Features of the Electronic Stethoscope (DS32A Digital Electronic Stethoscope)

\subsection{Electronic Stethoscope Arrays}

Lung auscultation provides the capability for a non-invasive method of diagnosis for lung diseases. Lung sounds have been shown to contain spatial information by (Kraman, 1980). By capturing multiple source signals from the body simultaneously, the spatial 
information from lung sounds can be accessed to provide diagnostic information for the lungs.

One of the objectives of this thesis is to harness the ability to simultaneously capture the output from sensor arrays. Stethoscope auscultation involves the physician listening to many auscultation points on the chest and abdominal areas of a patient's body. At each of the auscultation points, patients are asked to inhale and then exhale. This procedure introduces error in stethoscope auscultation as patients generally breathe differently between breaths. With mucus related respiratory diseases, in one breath an obstruction may impeded a patients breathing but in another breath, with a change in posture or a natural clearing of the mucus, breathing may be normal for an instant in time. This situation may drastically affect the physician's diagnosis for the patient. Also, there is a known psychological condition that patients may experience which is to seek positive reinforcement from the physician. This means that patients may constantly try to overcome their disease by forcing themselves to breath normally in order to receive acceptance from the physician (Carlson, Buskist, Enzle, \& Heth, 1997). This may not allow the physician to detect any diseases within the patient. These limitations to stethoscope auscultation can be eliminated with an array of stethoscopes and a computer that requires the patients to only breathe once.

\subsection{Adaptive Filters}

Adaptive filtering is widely used in order to identify the behaviour of an unknown system as a signal passes through it. Generally, such filters assume that the behaviour of the unknown system is linear and implement a Finite Impulse Response (FIR) filter and 
observe its transfer function. An FIR filter is constructed using a set of delays, coefficients, and other primitive operators (adders and multipliers). By passing a sampled input signal through an FIR filter, the output of the system with respect to any input known as the transfer function can be obtained. The maximum propagation delay of the input signal can be determined from the systems impulse response by selecting the filter coefficient with the highest magnitude and dividing by the sampling frequency. Peak frequencies can be extracted using a peak detector that analyzes the system transfer function for slopes that approach zero. Areas can also calculated for the system transfer function curves using fundamental calculus or more promptly using MATLAB. The transfer function of the system can also yield a frequency response of a variety of filters depending on the values of the FIR filter coefficients such as a: low pass, high pass, or a band pass filter.

The key to an adaptive filter is to provide the FIR filter with the ability to adjust its filter coefficients based on a coefficient update algorithm. There are many configurations of adaptive filters for signal separation and system identification. No matter how the adaptive filter is setup, the principle operation of an adaptive filter remains the same. That is, a signal is passed through the filter one sample at a time. This is known as an iteration of the algorithm. With each iteration of the algorithm, the coefficients of the filter are updated and the output of the filter is compared to a reference signal. When the error between the output of the filter and the reference signals is minimized or there is no data available to be passed through the adaptive filter, the algorithm is completed. A 
block diagram of an adaptive filter being used to extract a fetal Electro Cardio Gram (ECG) signal from a maternal ECG signal is shown in Figure 5 below.

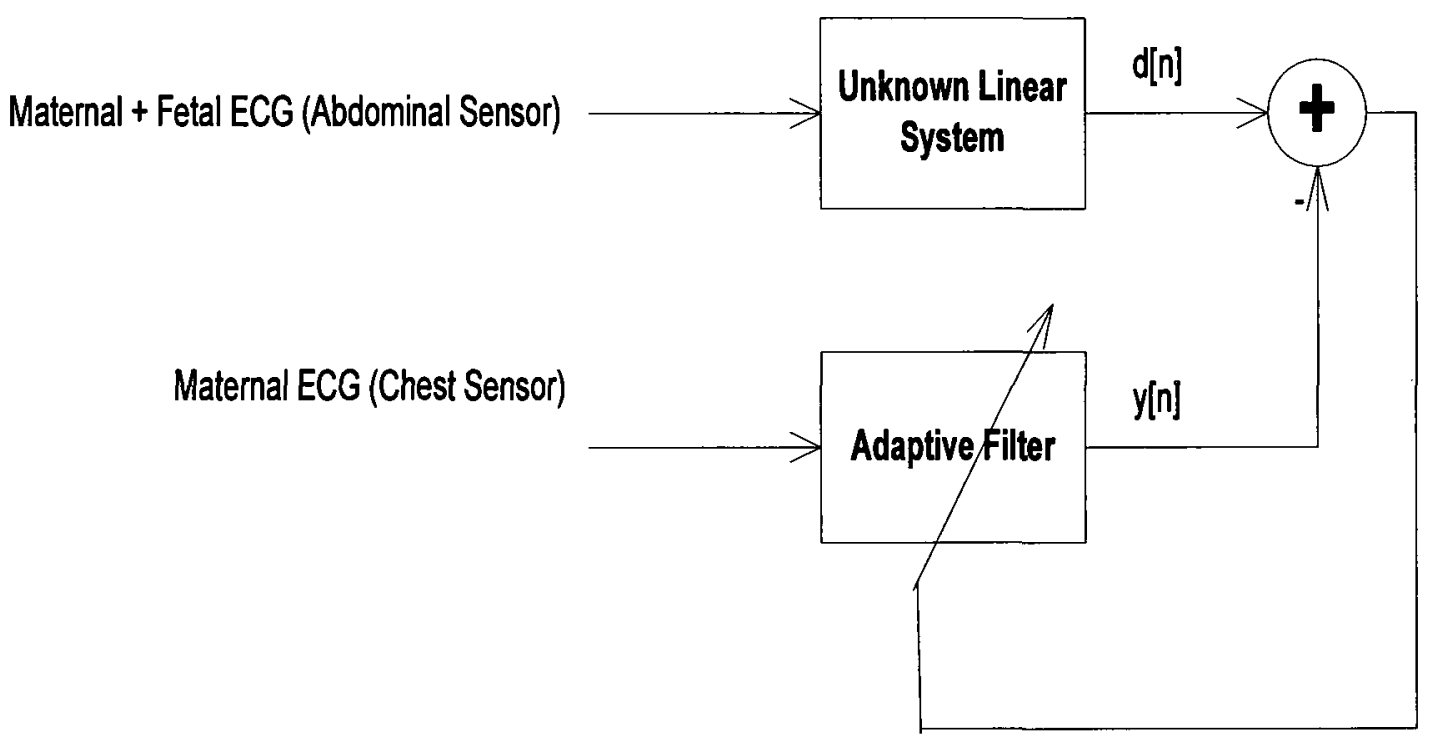

e[n] Fetal ECG

Figure 5 - Extracting Fetal ECG from Maternal ECG

The input signals into the system illustrated in Figure 5 are gathered using two electrodes placed on an expecting mother's chest surface over the heart and on the abdomen over the fetus. The maternal and fetal ECGs may be represented as the desired signal illustrated as $d[n]$ above. The adaptive filter will attempt to match the reference signal (maternal ECG) to the desired signal $d[n]$. Using this algorithm, the output of the adaptive filter $y[n]$ will never equal $d[n]$. The adaptive filtering algorithm will try and minimize the error between $y[n]$ and $d[n]$ and then stop. When this occurs however, the error signal $e[n]$ should be the exact difference between both input signals, which is the fetal ECG. Therefore, the desired result of the adaptive process is the error signal. In the arrangement shown above, the system that the unknown linear system represents is 
known. The focus of this thesis is to determine an FIR filter to describe the unknown linear system. This can be accomplished by feeding the same input signal into the unknown linear system and the adaptive filter as shown in Figure 6 below (Haykin, 2002).

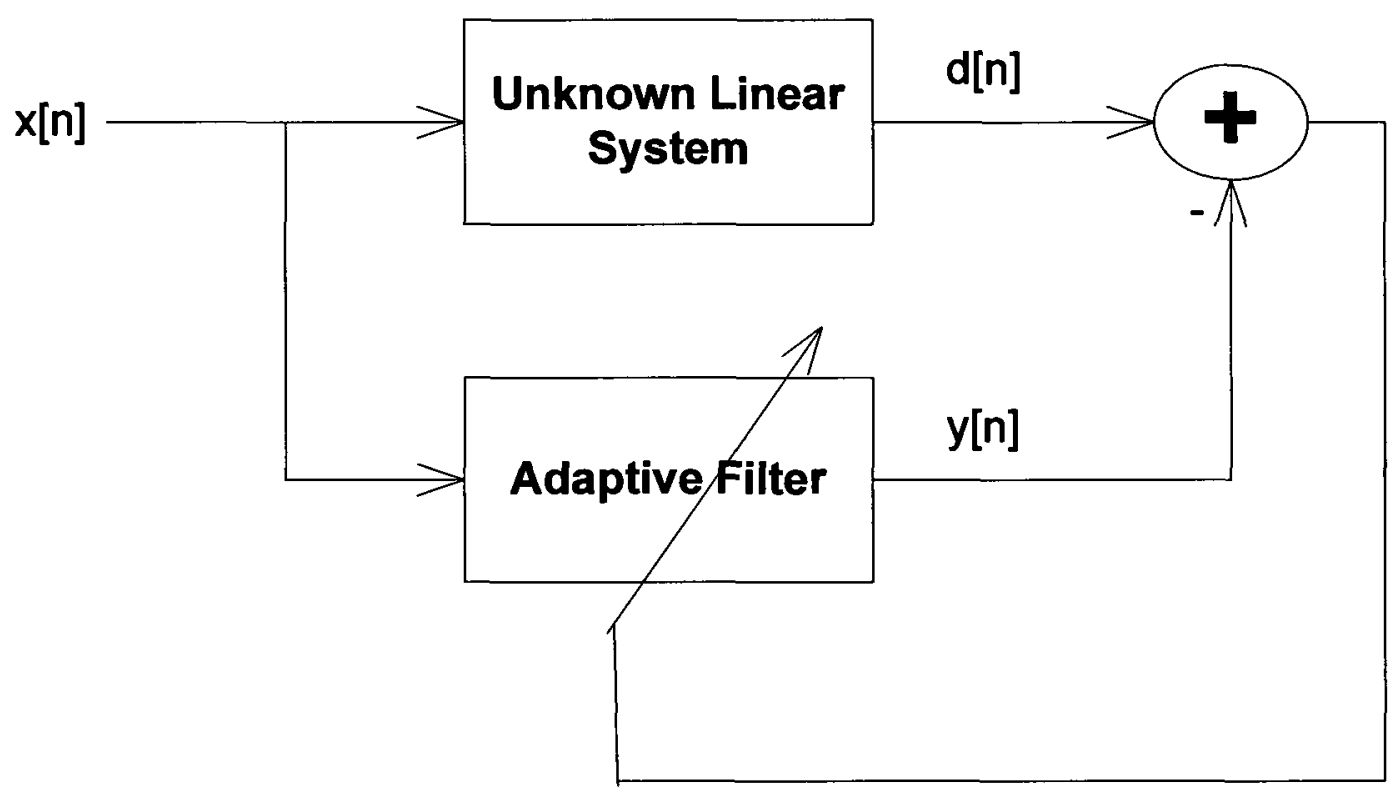

$\mathrm{e}[\mathrm{n}]$

Figure 6 - Identify Unknown System Using and Adaptive Filter by Feeding in the Same Input Signal

\subsection{Lung Models}

Many respiratory system models utilize foam or gelatine materials (McKee, 2004). These models are homogeneous and do not account for the varying materials within the chest such as those present in bones, organs, and tissues. This thesis attempts to account for the branching of airways into each lung but keeps the homogeneity attribute of previous lung models developed in our research group at Carleton University in the Department of Systems and Computer Engineering. Adaptive filtering will treat the chest of a patient as a "black box" thus ignoring the physiological characteristics of the chest and focusing 
more on the propagation paths that the input signal follows. Figure 7 and Figure 8 are illustrations of existing lung model concepts.

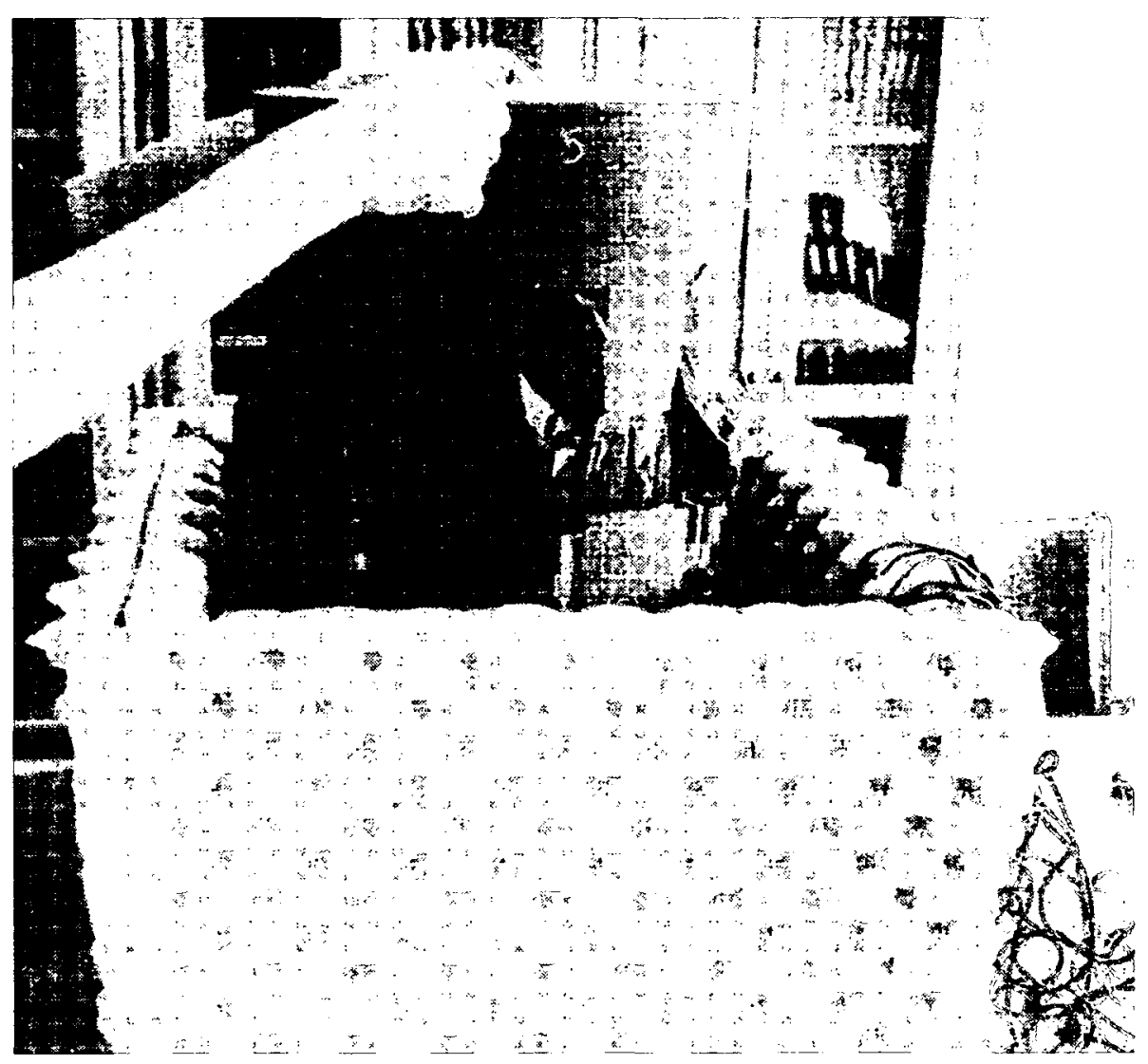

Figure 7 - Example Lung Model (Reproduced from (McKee, 2004))

The first model proposed by (Mckee, 2004) introduced in Figure 7 above was constructed using a hollow plastic cylinder lined with foam. 


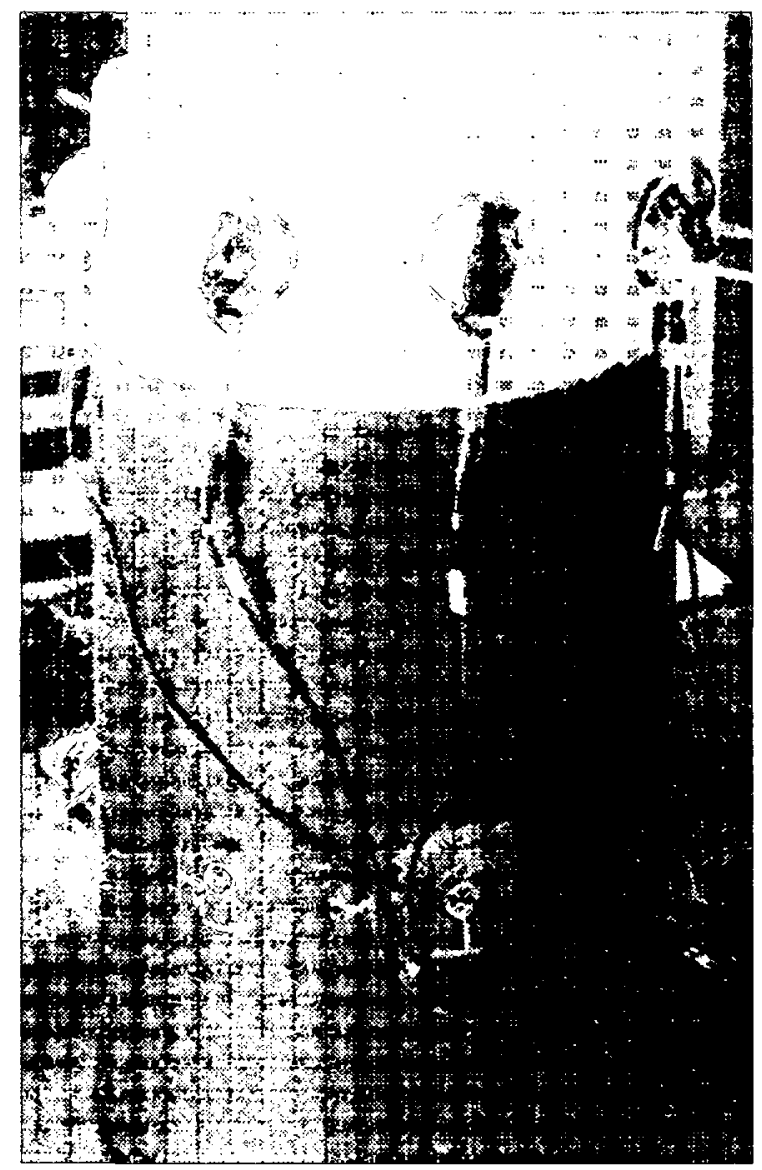

Figure 8 - Example Lung Model (Reproduced from (McKee, 2004))

The second model illustrated in Figure 8 was built using gelatine in order to form a solid cylinder. The gelatine enabled comparable speeds of sound within the cylinder with those of the human body that average around $36 \mathrm{~m} / \mathrm{s}$ in the chest.

The lung phantom model presented in this thesis attempts to extend the ideas formed in the construction of the model in Figure 7. 


\subsection{Breathing Manoeuvres}

This section discusses various breathing techniques that many people use to reduce the effects of Chronic Obstructive Airways Disease (COPD) on their lives. The medical instrument discussed in this thesis was designed to detect the movement of mucus within the respiratory system. Changes in posture or breathing techniques can help alleviate the effects of mucus by shifting it within the respiratory airways.

\subsubsection{Pursed Lip Breathing Technique}

This technique main purpose is to reduce the shortness of breath by reducing the frequency of breathing making each breath more effective. It also improves ventilation, releases trapped air in the lungs, keeps airways open longer and decrease the work of breathing, and moves old air out such that new air can enter the lungs. The technique

requires the following 4 steps: 1) Relax the neck and shoulder muscles, 2) Inhale slowly through the nose with the mouth closed for 2 seconds, 3) Picker or "purse the lips, and 4) Exhale slowly through the "pursed" lips for 4 seconds.

\subsubsection{Diaphragmatic Breathing}

The diaphragm is the most effective muscle in the breathing process. People with COPD however may have a dysfunctional diaphragm due to trapped air within the lungs that force the diaphragm down. The neck and chest muscles must then work harder to maintain efficiency. Diaphragmatic breathing aims to: strengthen the diaphragm, decrease the work of breathing by slowing the breathing rate, and decrease oxygen demand. Three steps are involved: 1) Lie on the back on a flat surface with knees bent 
and head supported, 2) Place hands on the upper and lower chest areas and inhale slowly with your nose, and 3) Tighten stomach muscles and exhale using "pursed" lips.

There are many more positions to reduce shortness of breath and strengthen the diaphragm in the sitting, standing, and resting positions (Fishleder \& Rothner, 2008).

\subsection{Current Lung Monitoring Technologies}

Many technologies using a variety of methods have been developed to monitor respiration. This section will mention some of the most common techniques and describe two methods related to acoustical lung imaging that apply similar principles to those used in this thesis.

\subsubsection{Positron Emission Tomography (PET)}

Positron Emission Tomography is widely used to detect the presence of cancer within the body. PET scans are diagnostic tests that use a small and safe dose of a radioactive compound known as a "tracer" that emits gamma rays which are detected using an imaging scanner. PET scans can detect biochemical processes in the body that may indicate the formation of diseases before there are any anatomical growths. This method is regarded as a more improved cancer detection system when compared to MRIs and CT scans described in the following sections due to its ability to detect the onset of cancer before the formation of tumours (Webster, 1998). 


\subsubsection{Magnetic Resonance Imaging (MRI)}

Magnetic Resonance Imaging (MRI) uses a magnetic field to align the nuclear magnetization of hydrogen atoms in water in the body and the recovered magnetic field signal can be manipulated by additional magnetic fields to build up enough information to construct an image of the body. MRI performs very well in soft tissues with large water content (Webster, 1998).

\subsubsection{Electrical Impedance Tomography (EIT)}

Electrical Impedance Tomography (EIT) uses the principles of varying conductivities within the body and measured electrical potentials to develop images of the inside of the body. Typically, EIT involves the attachment of electrodes to the surface of the body in and around the area to be imaged and small electrical AC currents are applied (Webster, 1998).

\subsubsection{Acoustic Imaging of the Human Chest}

This section describes two lung monitoring methods that apply the principles of acoustics. The first technology is still in research stages while the second is available as a commercial product.

The first system developed by (Kompis, Pasterkamp, \& Wodicka, 2001) uses an array of microphones attached to the anterior and posterior auscultation points of the chest to record breath sounds. The breath sounds are then post-processed to form volumetric 
images based on sound propagation and source estimation of the lungs as air enters and exits.

The algorithm uses a least-squares fit to estimate a sound source for each microphone assuming a uniform sound propagation throughout the thorax (speed of sound, damping factor). The imaging algorithm follows six underlying principles:

1. The algorithm must be robust with respect to the acoustic properties within the thorax.

2.The algorithm should not rely on the measurement of the time of arrival of lung sound components

3.The algorithm should provide three-dimensional data sets

4. The resulting images should be intuitively interpretable

5.Given current sensor technology, the number of microphones should be limited in number.

6. The algorithm should be robust with respect to missing microphones or noisy data in individual microphones

These principles are applied to form greyscale images of the thorax. This system is limited because of the sound source complexity of the thorax. Due to a large number of sound sources within the thorax, there is difficulty in isolating the coordinates of the desired sources due to large amounts of noise. Inaccurate delay estimation results are yielded by the algorithm and the lung images appear grainy. 
The second system was developed in conjunction with General Electric (GE) and Deep Breeze Inc. and is currently available on the healthcare market. The technology is known as Vibration Response Imaging (VRI). The technology was aimed at diagnosing and monitoring problems with intra-thoracic air flow. Because of its monitoring capabilities, VRI can be used to rate the effectiveness of lung treatments.

VRI measures vibrations on the posterior thoracic area from airflow generated through simple breathing (requiring only $2-3$ patient breaths), to create a radiation free, dynamic, real-time structural and functional gray scale image of the respiration process (Rajanala, Jean, Kushnir, Dellinger, \& Parrillo, 2005).

All of the technologies aforementioned can be used to monitor the respiratory system for disease. Many of them use expensive techniques that use complex algorithms. This medical instrument utilizes low cost and already widely used stethoscopes in order to build and improve existing medical equipment. 


\section{Chapter 3 - Measurement Apparatus Setup}

This chapter describes the instrumentation aspects of a medical instrument developed in this thesis that measures changes in the distribution and density of lung fluid. The instrument uses a sound signal to excite the respiratory system and measures it on the surface of the chest using an array of electronic stethoscopes in order to develop a sound propagation model of the chest. 


\subsection{Data Acquisition}

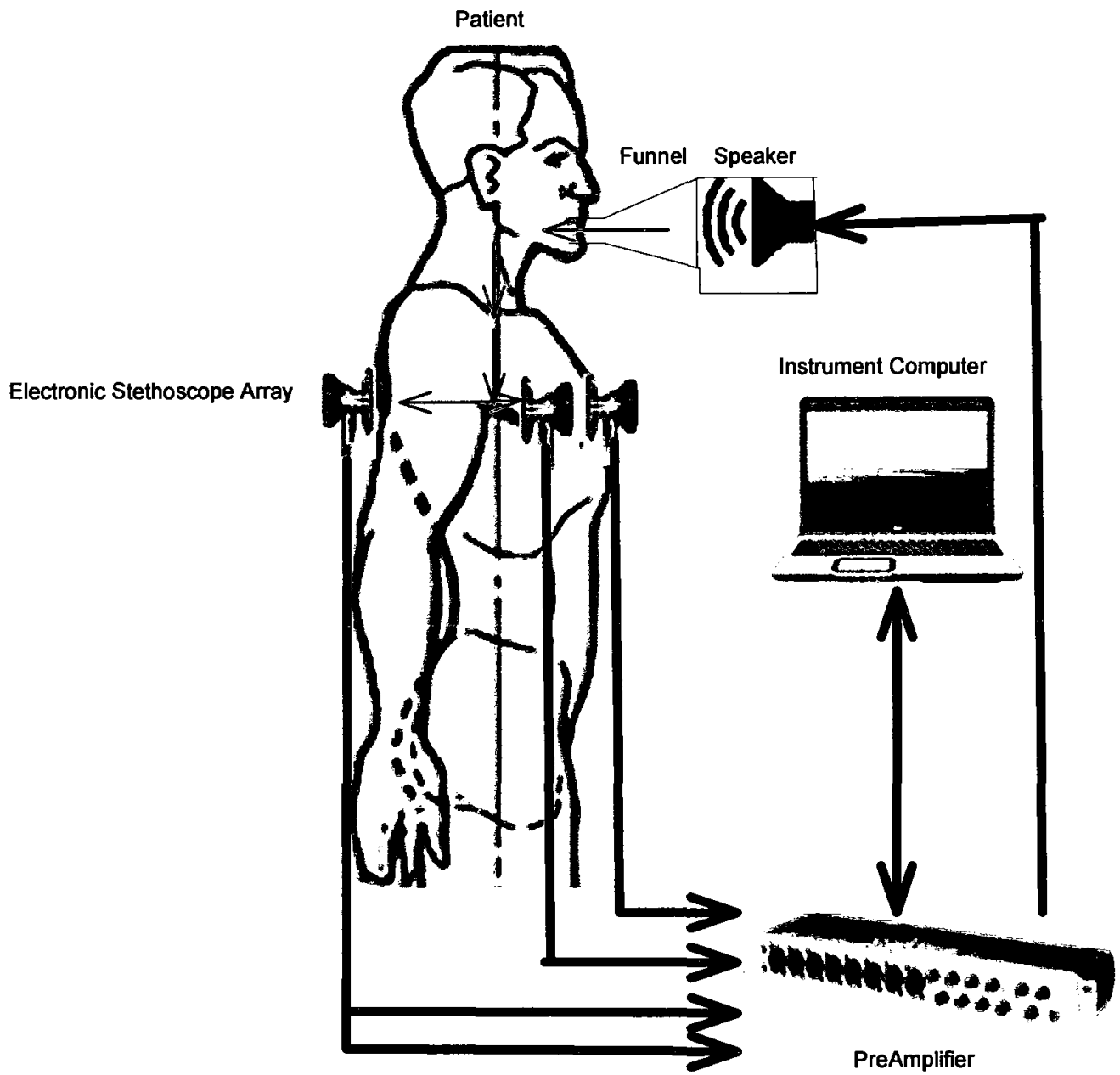

Figure 9 - Sketch of Measurement Apparatus and Setup on a Patient

The medical instrument setup on a patient in Figure 9 was designed to emit a sound into a patient's mouth and measure the sound on the anterior and posterior surfaces of the chest using an array of stethoscopes placed on 4 of the lung auscultation points ( 3 and 4 shown in Figure 3). For the sound emitting component of the data acquisition process a White Gaussian Noise (WGN) input signal spread over the frequency spectrum of $0-4 \mathrm{kHz}$ was generated using MATLAB and stored into the main memory of a computer (HP DV9210CA). The input signal was converted into an analog signal and outputted through the computer's sound card using a Conexant (CX20549) digital signal processor chipset 
where it was sampled by an external device that detects and strengthens weak signals known as a preamplifier (PreSonus FirePod) with a sampling frequency of $8 \mathrm{kHz}$. The preamplifier then outputs all of its acquired signals serially back to the computer through a firewire port.

As the preamplifier subjected signals to various amplifications and filtering conditions, it was important to pass the input signal from the computer through the preamplifier such that it was exposed to the same conditions as the acquired signals. If the input signal had not been passed through the preamplifier and the acquired signals were drastically changed by the preamplifier, algorithms designed to compare the signals such as crosscorrelation would not be valid because the signals would be too dissimilar and produce erroneous and inconsistent results.

The input signal was output from the preamplifier into a patient's mouth via a loudspeaker with a funnel attached to its end. Following traversal through the patient's body, the input signal was captured by an array of 4 electronic stethoscopes (ThinkLabs DS32A) aligned to auscultation points 2 and 3 on the anterior and posterior of the patient. In order to align the stethoscopes in the array over their respective auscultation points, a harness was developed. The harness was constructed using the materials in Table 3. 


\begin{tabular}{|c|c|}
\hline Material & Quantity \\
\hline 1'x6' tow strap & 2 \\
\hline 3'x3' tow strap & 1 \\
\hline Brass Cuffs & 4 \\
\hline Nuts and Bolts & N/A \\
\hline
\end{tabular}

Table 3 - Materials used to Construct the Stethoscope Array Harness

The three inch strap was used to fasten the stethoscope array to the surface of the body and allowed adjustment for varying chest circumferences. Slots were cut into the brass cuffs such that they could encompass the stethoscope head of each stethoscope. The three inch strap was then laced through the brass cuffs. The one inch straps were shortened and sewn to the three inch strap in order to create adjustable shoulder straps to account for varying body heights. The end result illustrated in Figure 10 was a fully adjustable stethoscope harness that could be attached to nearly anything (in this case a human participant). 
Figure 10 - Fully Adjustable Stethoscope Array Harness Attached to a Human Participant

The signals captured by the array were outputted from the stethoscopes to 4 different channels on the preamplifier and sent to the computer via the firewire port. The signals were digitized by a Texas Instruments digital signal processor (TIXIO2200A), and placed into the computer's main memory. The signals were removed from main memory and written to the computer's hard drive by the developed software (using the MATLAB language). Figure 11 illustrates the sound emitting, acquisition and processing instrument with an unknown system as the measurand. 


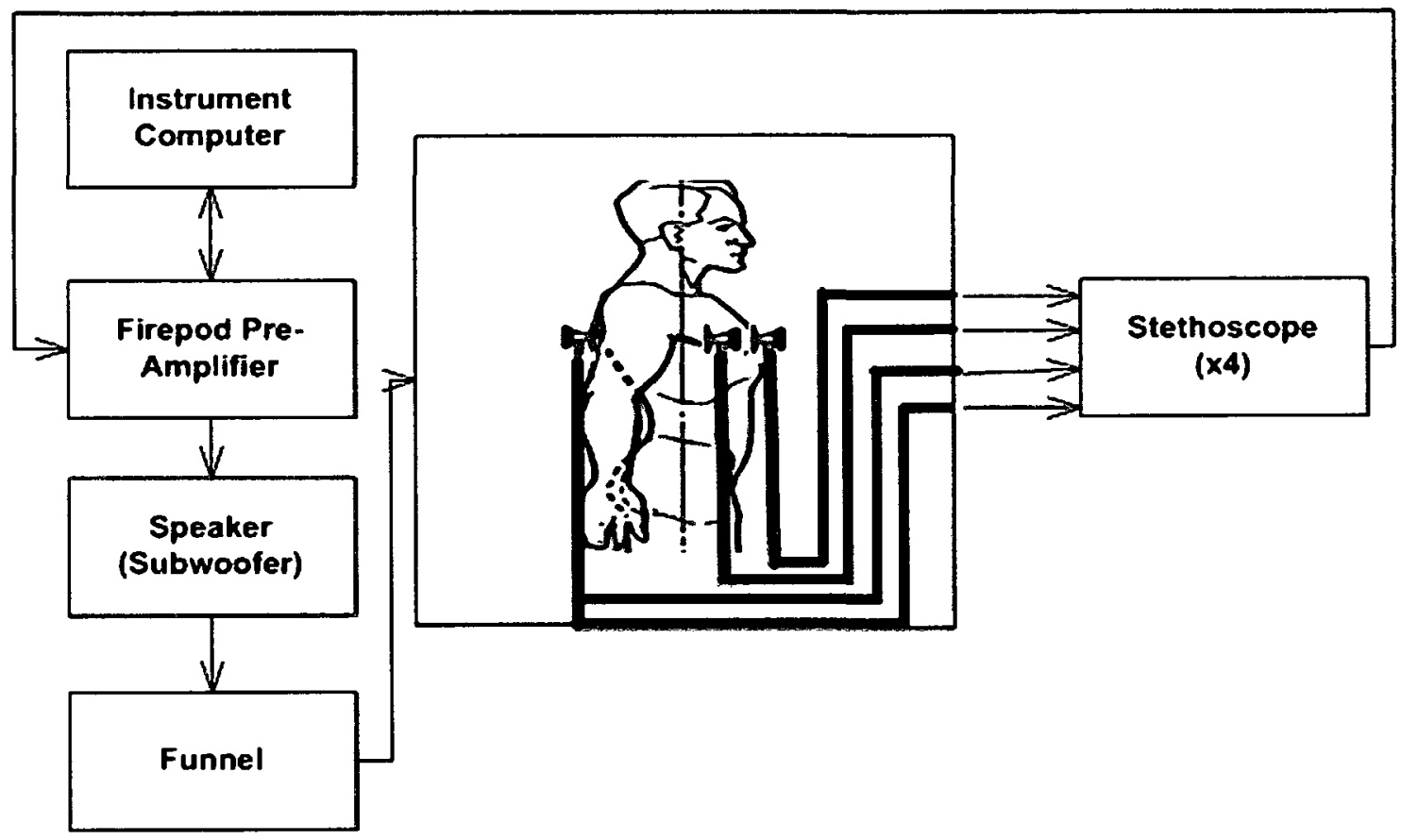

Figure 11 - Block Diagram of the Medical Instrument

Figure 12 shows pictures of the actual medical instrument off the shelf components with the stethoscope array and harness attached to a participant. 


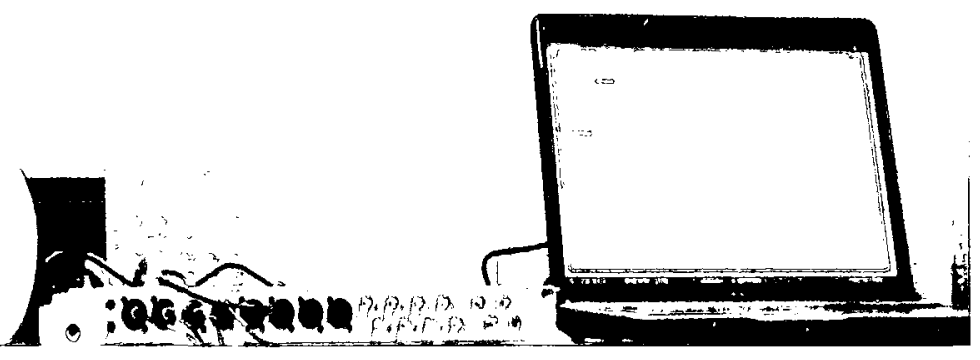

Sound Generator

Pre-Amplifier

Computer

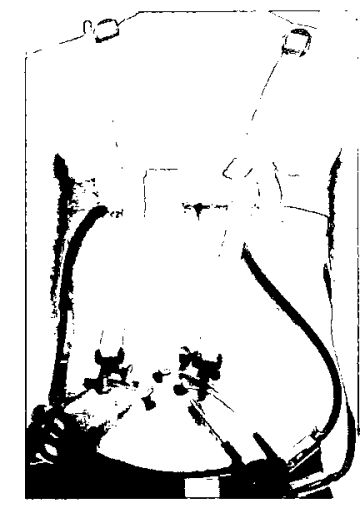

Stethoscope Array and Harness Attached to a Participant

Figure 12 - Actual Off the Shelf Components of the Medical Instrument

Table 4 summarizes the components used to build the instruments sound emitting and recording interfaces.

\begin{tabular}{|l|c|}
\hline \multicolumn{1}{|c|}{ Component } & Quantity \\
\hline Logitech Z3 Subwoofer (23 W) & 1 \\
\hline Firepod 8 channel pre-amplifier & 1 \\
\hline TIXIO2200A Firewire Card & 1 \\
\hline ThinkLabs DS32A Electronic Stethoscope & 4 \\
\hline HP Pavillion DV9210CA Laptop & 1 \\
\hline
\end{tabular}

Table 4 - Components used to construct the sound emitting and recording apparatus 


\subsection{Data Processing}

This section describes techniques and algorithms used to process the acquired sound data.

\subsubsection{Instrument Calibration}

Due to the fact that the delay between the input and output signals was an important parameter described in a later section, the instrument required a calibration step to account for the offset delay caused by the measurement apparatus. It is shown in Figure 13 that within the off the shelf components used to build the instrument, there are many internal electrical components that delay signals. Each Off the Shelf (OTS) component includes a number of internal components that are not necessary for this instrument but are used to save time for prototype development. The limitation is that a calibration process is required to account for the overlooked component delays. The preamplifier used in the instrument sampled signals from the stethoscopes at $44.1 \mathrm{kHz}$. This allowed for a calibration accuracy of $22.6 \mu \mathrm{s}$. 


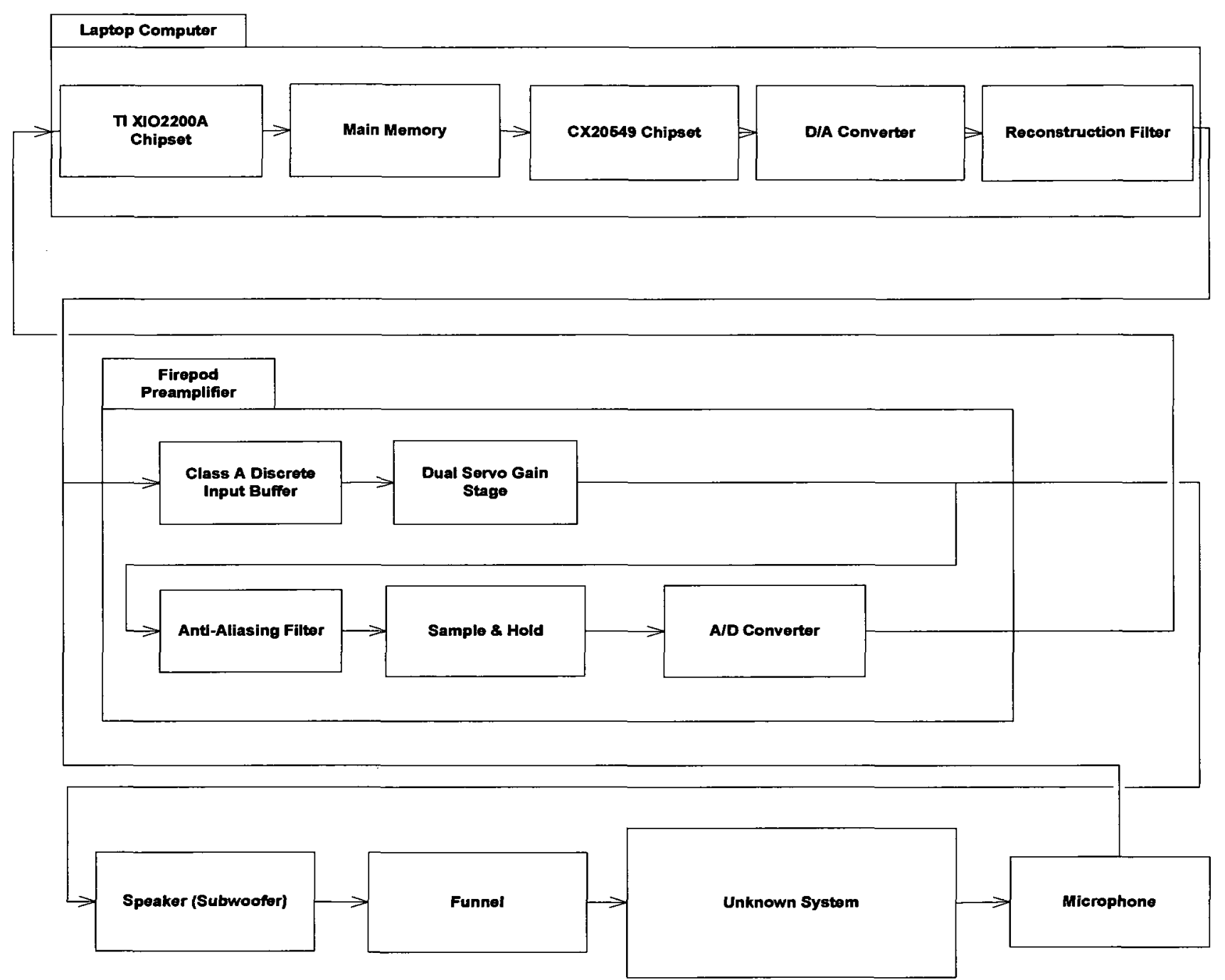

Figure 13 - Medical Instrument Block Diagram of Off the Shelf Components with Internal Components 
The calibration of the instrument was performed as described in the following paragraphs.

The first step was to determine the delay of the signal travelling through the pre-amplifier and back into the computer. In order to do this, the input signal was connected to the first channel of the pre-amplifier and then feed back into the second channel shown in Figure 14.

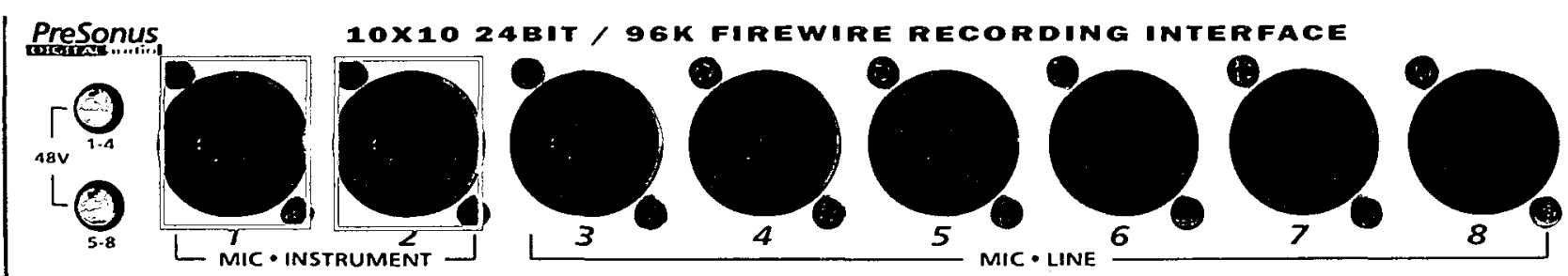

Figure 14 - Face Panel of Firepod Preamplifier. Boxes Show Channels 1 and 2 of the Preamplifier.

The signal captured by the second channel of the pre-amplifier was then sent through the firewire port of the preamplifier to the computer. Figure 15 illustrates a simplified diagram of the pre-amplifier and the laptop computer without their internal components.

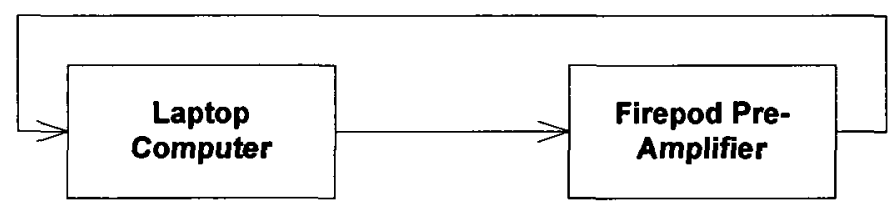

Figure 15 - Detecting the Delay of the Pre-Amplifier

The delay of the input signal from the laptop computer and the signal recovered from the pre-amplifier was determined using the NLMS algorithm and the NLMS algorithm was tested using standard cross-correlation which also compares two signals and can calculate the maximum delay between them. By looking at the coefficient values of the NLMS adaptive filter shown in Figure 16, the coefficient with the highest amplitude designates 
the maximum delay between the input signal and the signal recovered by the preamplifier. The coefficient number indicates the number of samples that the input signal is delayed from the recovered signal. The time delay between the two signals can be calculated by simply dividing coefficient index by the sampling frequency as in Equation 1 where $\mathrm{c}$ is the coefficient number and $\mathrm{x}$ is the sampling frequency.

$$
\text { c sample } * \frac{1 s}{x \text { sampies }}
$$

In this case, the maximum delay between the two signals was 484 samples. Therefore, with a sampling frequency of $44.1 \mathrm{kHz}$, the delay between the two signals was 10.975 ms.

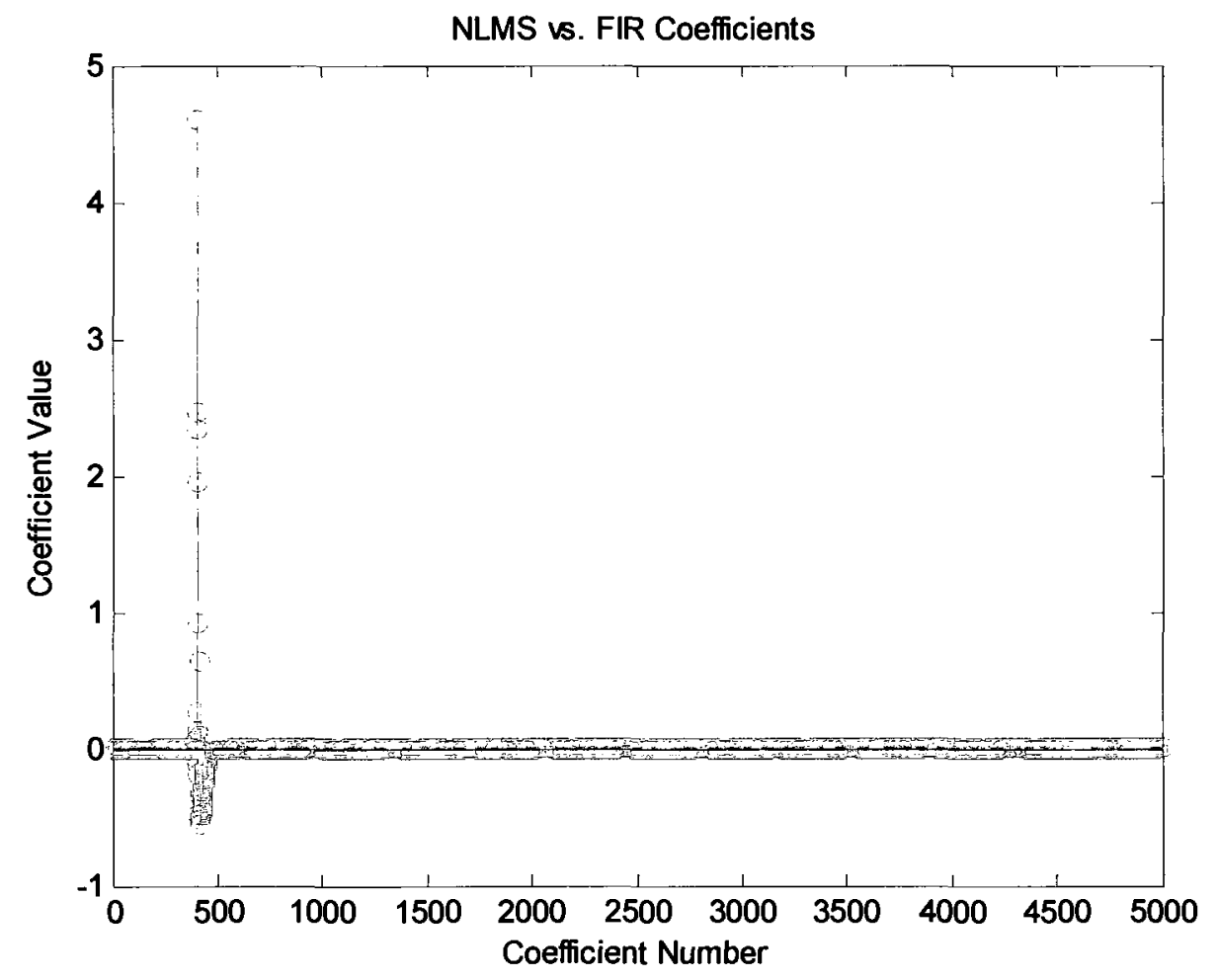

Figure 16 - NLMS Coefficients upon Convergence of the Filter 
The second stage that the input signal travelled through was into a speaker (subwoofer). Therefore, the total delay includes the laptop computer, pre-amplifier, and the speaker amplifier (See Figure 17).

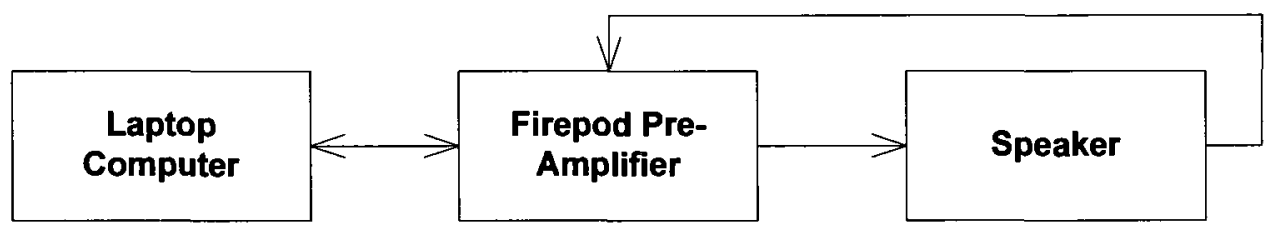

Figure 17 - Detecting Delay of the Speaker

The speaker delay consisted of an external amplifier and a transducer both with individual delays. The speaker had an external amplifier delay which was measured by feeding the input signal from the computer back to the pre-amplifier through a line out port on the speaker. The delay of the pre-amplifier was then subtracted from the total delay show in Figure 18 between the input signal and the recovered signal. The speaker amplifier delay was determined to be $0.5 \mathrm{~ms}$. 
NLMS vs. FIR Coefficients

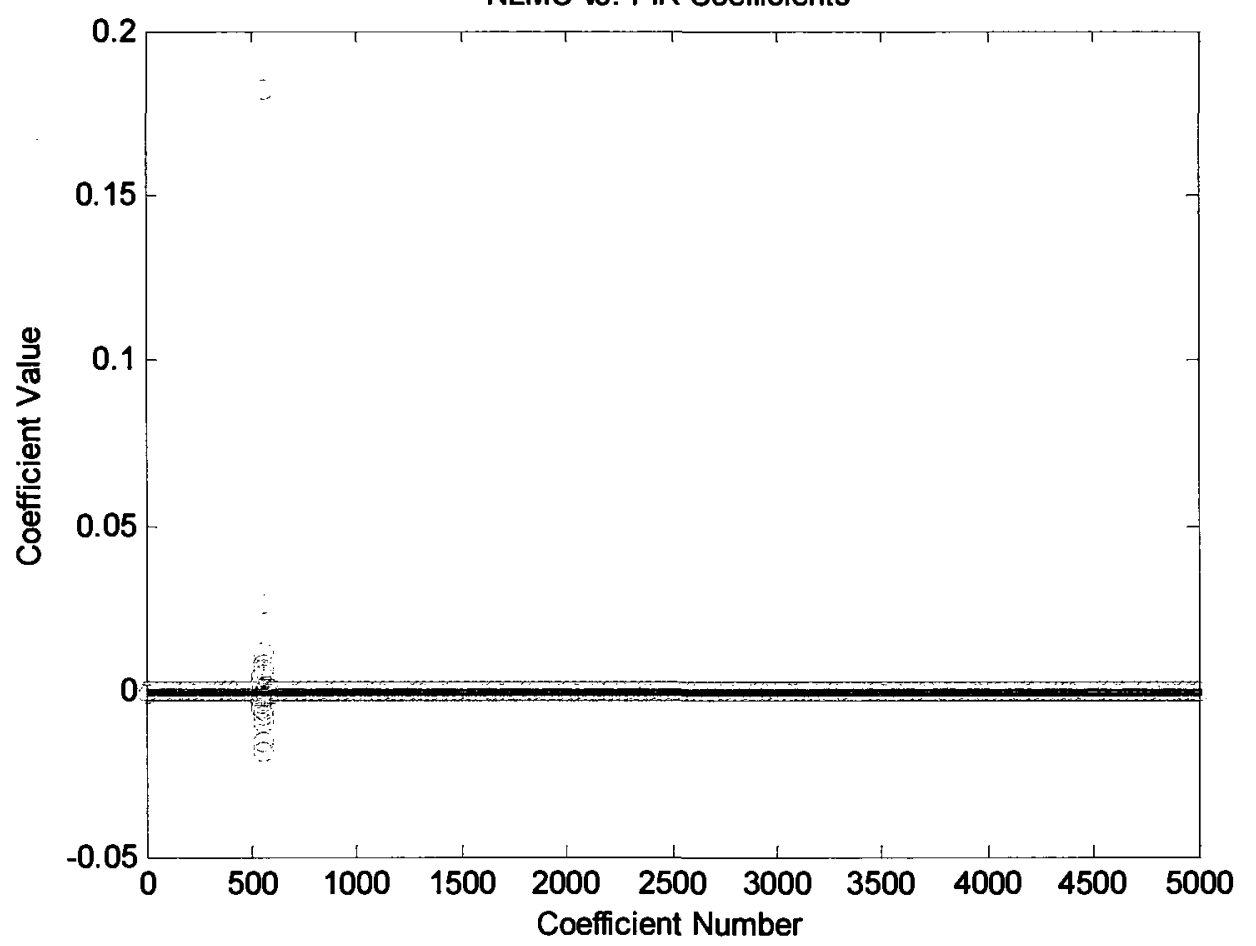

Figure 18 - NLMS Coefficients Showing the Delay between the Laptop Computer, Pre-Amplifier, and the Speaker Amplifier

Next, the delays of the transducer component of the speaker, funnel, and microphone were all determined simultaneously (see Figure 19).

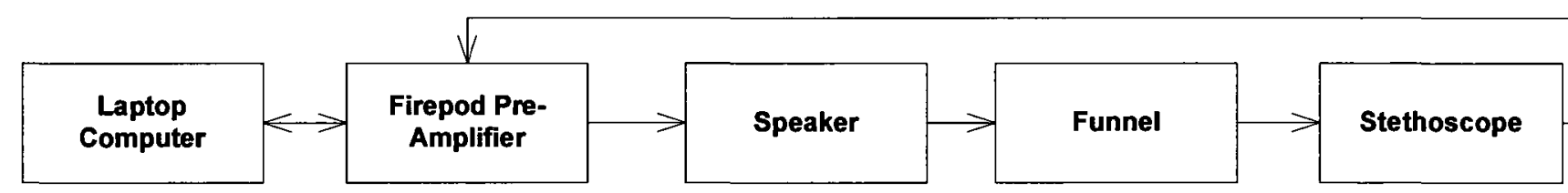

Figure 19 - Detecting Delay of the Speaker Transducer, Funnel, and Stethoscope

This was done by placing the microphone (stethoscope) as closely and as tightly as possible to the end of the funnel and recording the input signal. The delay of all three components was calculated to be $2.273 \mathrm{~ms}$ and the NLMS coefficients plot is shown in Figure 20 below. In order to estimate the delay in the funnel, it was assumed that the 
speed of sound within the funnel was approximately that of air $(343 \mathrm{~m} / \mathrm{s})$; this yields a funnel delay of $0.72 \mathrm{~ms}$ for a measured length of the funnel of $0.25 \mathrm{~m}$ (Equation 2). In fact this assumption may be inaccurate because the plastic of the funnel provides a faster channel for the sound signal.

$$
t=\frac{\pi}{w}=\frac{0.25}{343} \div 1000=0.72 \mathrm{~ms}
$$

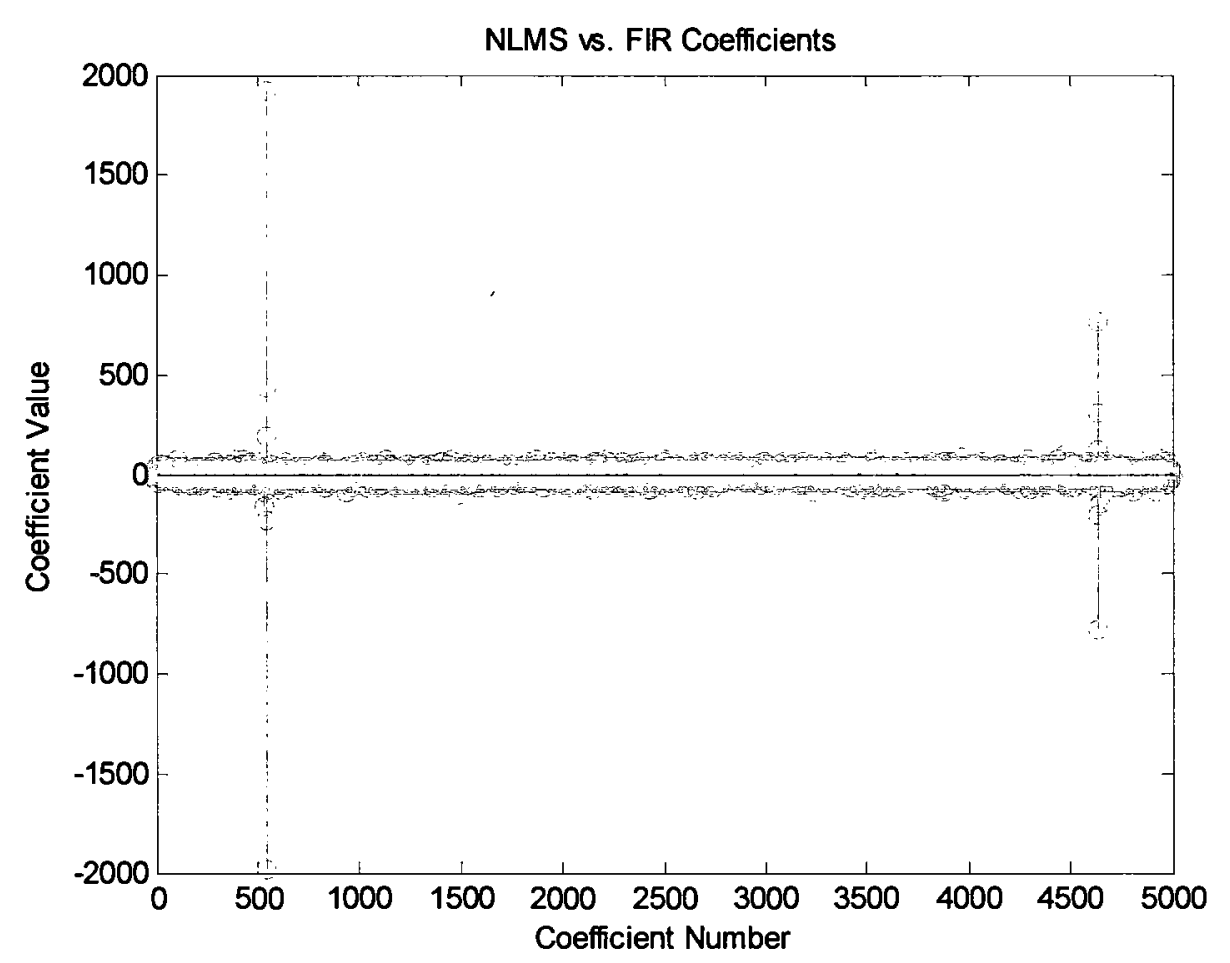

Figure 20 - NLMS Coefficients showing the Delay between the Speaker Transducer, Funnel, and Stethoscope

\subsubsection{Algorithm Implementation}

The acquired data was processed using an adaptive filtering scheme. The input signal was passed through an adaptive filter and compared with the signal measured by the array of stethoscopes. The adaptive filter implemented the NLMS algorithm to update its 
coefficients using the coefficient update algorithm described by Equation 3 where $\mu$ is the step size, $w[n], e[n]$, and $I[n]$ are the coefficient, error, and input vectors respectively.

$$
w[n+1]=w[n]+2 \mu e[n] I[n]
$$

The length of the FIR filter was 1500 coefficients with the coefficient update algorithm used a step size $\mu=0.296$. The NLMS adaptive filter was implemented using the Signal Processing Blockset within MATLAB. The length of the signals used for analysis was 40000 samples or 5 seconds of data. Figure 21 is a block diagram of the data processing system setup that employs the NLMS adaptive filter setup for this project. In the figure $y_{c}(n)$ is the signal measured from a stethoscope placed on one of the auscultation points on the surface of the chest, $y_{m}(n)$ is the output from the NLMS adaptive filter, and $e(n)$ is the error signal between $y_{c}(n)$ and $y_{m}(n)$. The algorithm was executed for 40000 iterations over all measured data.

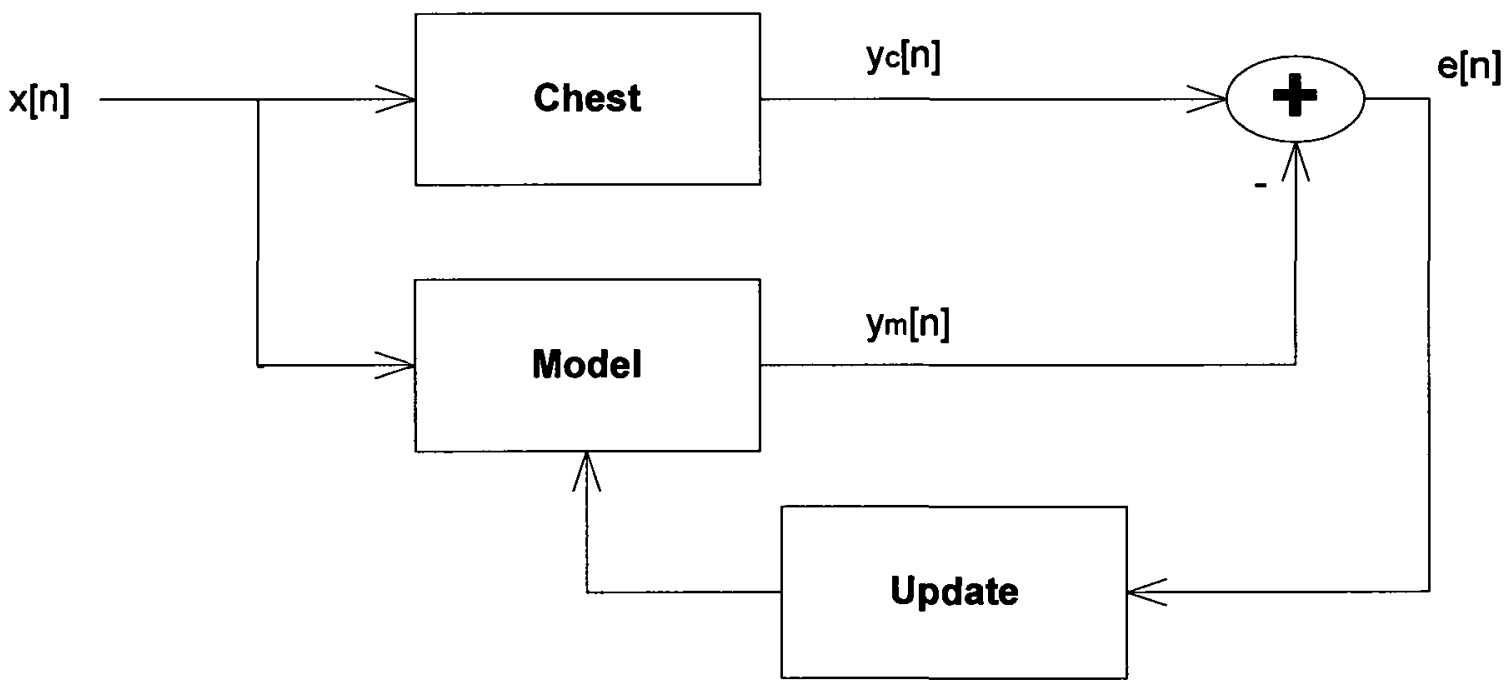

Figure 21 - Data Processing System Setup 
The result of the plotting the adaptive filter coefficients is the impulse response of the unknown system. The impulse response is useful for determining sound propagation delay and the transfer function of the unknown system. Changes in these parameters can then be used to determine the distribution and density of fluid within the lungs.

\subsection{Chapter Summary}

This chapter described the development of a medical instrument used to measure changes in the distribution and density of fluid within the respiratory system. The instrument used principles of sound emitting, acquisition, and processing to obtain the impulse response of an unknown system through adaptive filtering. Parameters such as sound propagation delay and the system transfer function can be extracted from the impulse response. The instrument is also capable of attaching to both phantom and human chests due to a fully adjustable harness that holds an array of electronic stethoscopes. 


\section{Chapter 4 - Simulation Results}

This chapter discusses simulations that were performed in order to select an appropriate input signal emitted by the medical instrument, adaptive filter parameters, and test the instruments data processing algorithms implementation and stability.

\subsection{Input Signal Selection}

The instrument's main function was to build a linear model for an unknown system (phantom or human chest). One of the results from the NLMS adaptive filtering algorithm was the systems impulse response used to build an audio transfer function that described changes to the input signal as it travelled through an unknown system and was measured by the array of stethoscopes. The frequency of the input signal defines what frequency information is relevant in the transfer function of the unknown system. As an example, if the frequency of the input signal spanned from $0 \mathrm{kHz}$ to $22.05 \mathrm{kHz}$ and the recovered signal only contained frequency components between $0 \mathrm{kHz}$ and $4 \mathrm{kHz}$, much of the lower frequency information would be masked because the transfer function would appear squished due to many high frequency components being zero. Figure 22 shows two transfer function plots that were created using measurements from a chest phantom. The plot on the left of the figure uses an input signal with a frequency span as in the example. The other plot has an input signal that has a frequency span between $0 \mathrm{kHz}$ and $4 \mathrm{kHz}$. It is evident that with the smaller frequency span, more information (a hidden peak) can be seen in the transfer function likely due to the algorithm used by MATLAB to plot the transfer function (specplot) and thus this frequency span was chosen for the 
input signal. This is likely due to the approximations used by MATLAB's specplot function. As the number of points in the frequency response calculation increases, for memory management purposes, some points must be discarded. Therefore, by focusing only on the frequency range of interest, a higher resolution in the frequency response can be obtained.
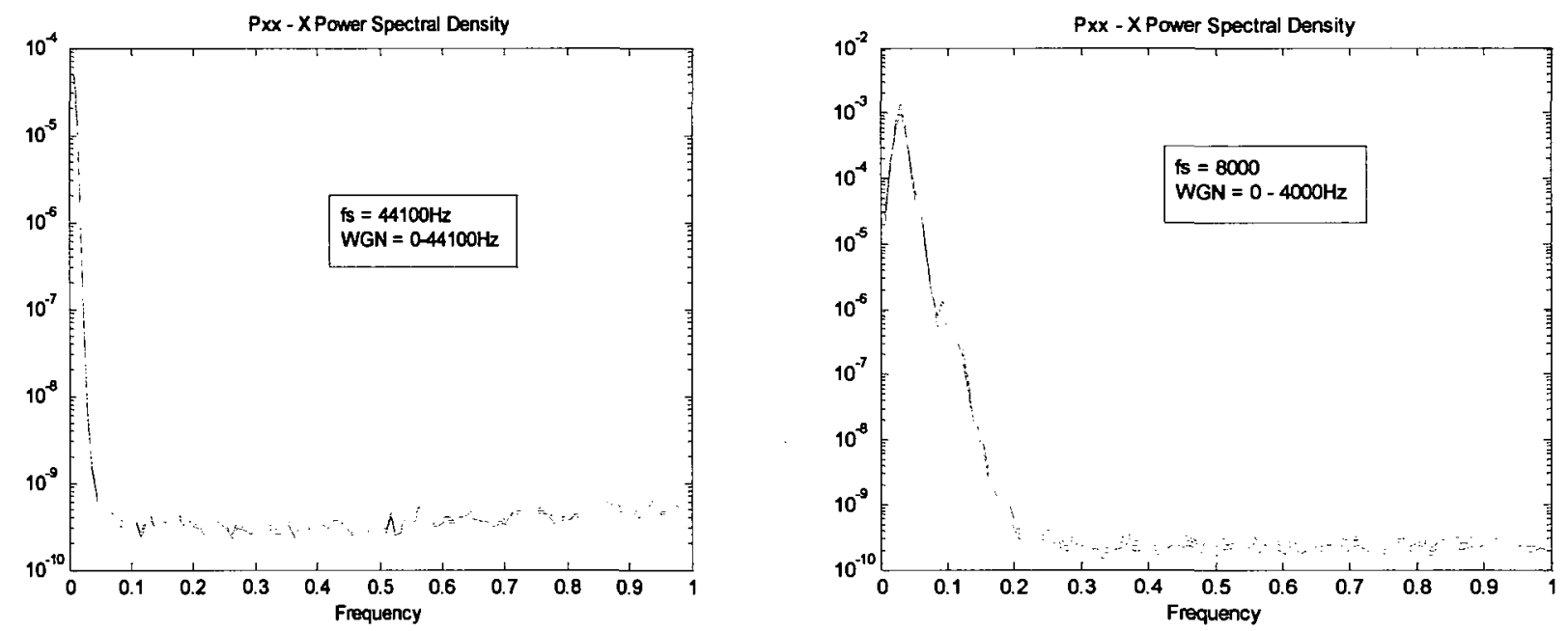

Figure 22 - Various Frequency Responses (Normalized Frequency $x \pi \mathrm{rad} / \mathrm{sample}$ ) of a Chest Phantom to Determine the Appropriate Input Signal Frequency Spread (where fs is the sampling frequency, WGN is White Gaussian Noise)

\subsection{Adaptive Filter Parameter Selection}

Two parameters had to be configured in order to use adaptive filtering to develop a model: the number of filter coefficients and the filter step size $(\mu)$. When the adaptive filter converges to produce a model of an unknown system's behaviour, the coefficients that form the system's impulse response should eventually reach zero and remain zero for every excess coefficient that is not required for the model. In reality, these coefficients however around zero but never remain constantly zero due to noise. Therefore, for both the phantom and human experiments for this project, a filter with a reasonable number of 
coefficients had to be built. Furthermore, the step size affects how the adaptive filtering algorithm adjusts the filter coefficients with each iteration. If the step size is too small, the adaptive filter may not have enough iterations due to a lack of data to converge to its lowest possible error value. Secondly, if the step size is too large, the adaptive filter may not converge at all and thus no model will be produced for the unknown system.

For each experiment performed in the next sections, test runs were performed such that, given a reasonable number of coefficients $(1500)$ and step size $(\mu=0.296)$ the adaptive

filter algorithm converged with a final relative error value on the magnitude of $10^{-6}$. The choice of the number of coefficients was driven by the requirement to have a sufficiently low final relative error. The choice of 1500 coefficients was approximately three times larger than the required amount seen in sample experiments. The value of $\mu$ as 0.296 was chosen to give the minimum final MSE. Its value was chosen using a bisection search technique however values close to 0.296 to result in an algorithm convergence although with slightly higher MSE's.

\subsection{Verification with Adaptive Filter Simulator and FFT}

An adaptive filter simulator was available as an educational tool developed under Dr. Rafik Goubran by Hung Xu in the Department of Systems and Computer Engineering at Carleton University shown in Figure 23. 


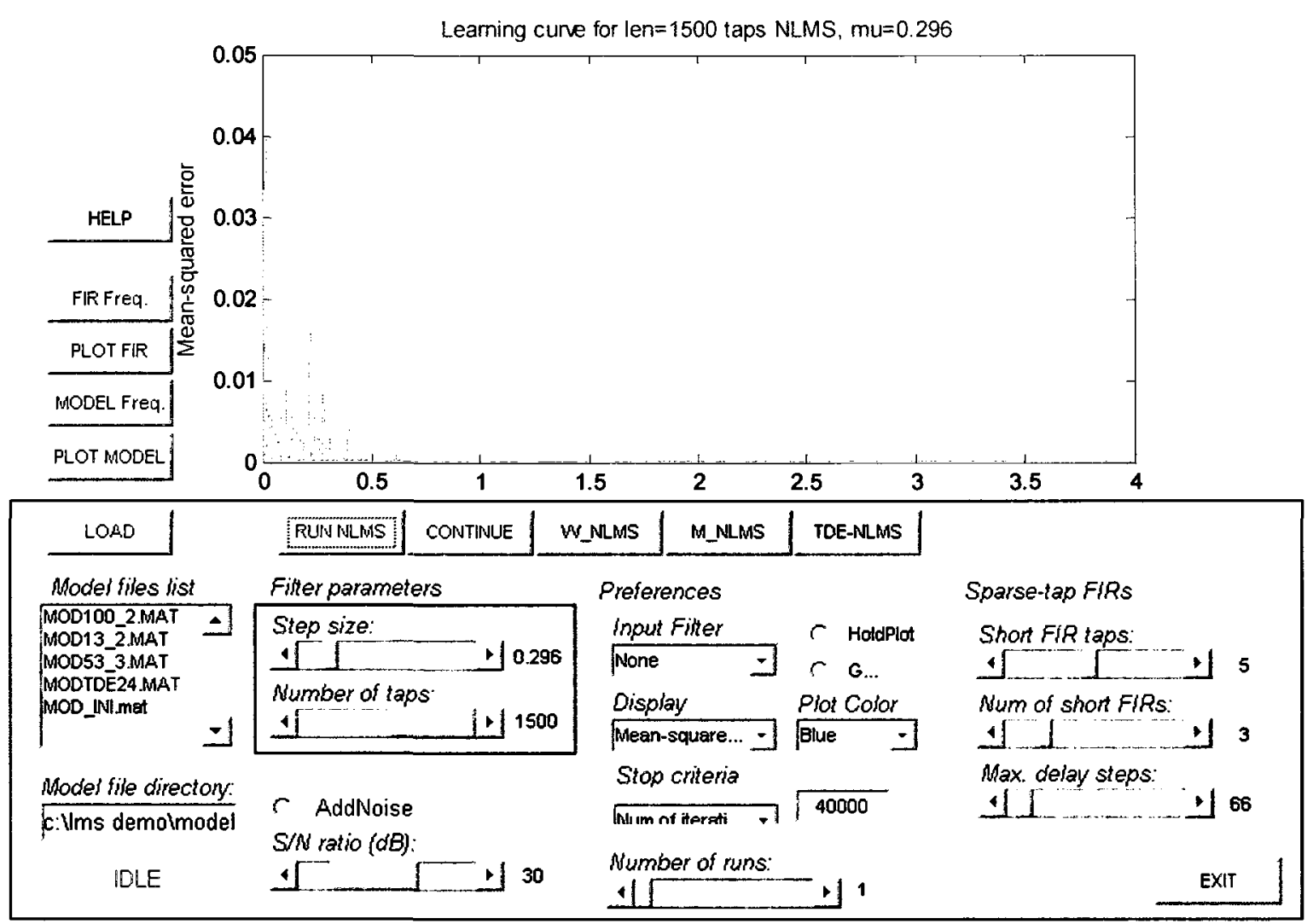

Figure 23 - Adaptive Filter Simulator Developed by the Department of Systems and Computer Engineering Carleton University.

The tool was capable of performing the NLMS algorithm with a WGN input sequence. The tool could be used to load a set of coefficients as an unknown system and perform NLMS to identify the system. The coefficients that the NLMS algorithm identified could be plotted with the coefficients of the unknown system for verification along with the Mean Squared Error (MSE) curve of the filter. The tool allowed the adjustment of filter parameters such as the step size, number of coefficients, and number of iterations of the algorithm among other parameters. With slight modifications the tool could also accept two sequences: an input sequence and a sequence measured from an unknown system (output sequence) to match the algorithm used in this project. The tool could execute the NLMS adaptive filtering algorithm using the data and develop a model. The model could then be compared to other implementations of the NLMS algorithm for verification. 
Test data were collected from one human participant. The participant was seated in an upright position while wearing the stethoscope harness. While breathing normally with their mouth surrounding the end of the funnel, the input signal was injected for 5 seconds into the participant's mouth and measured on their chest surface. This constituted one trial of the experiment. The data were entered into both the adaptive filtering data processing algorithm of the medical instrument and the adaptive filtering simulator. The models developed from each program were compared and shown in Figure 24. The results from the simulator were plotted in green over those of the data processing algorithm in red and it can be observed that both models are equivalent. The models should be equivalent as they are both implementing the same algorithm but constructed from two independent authors. 


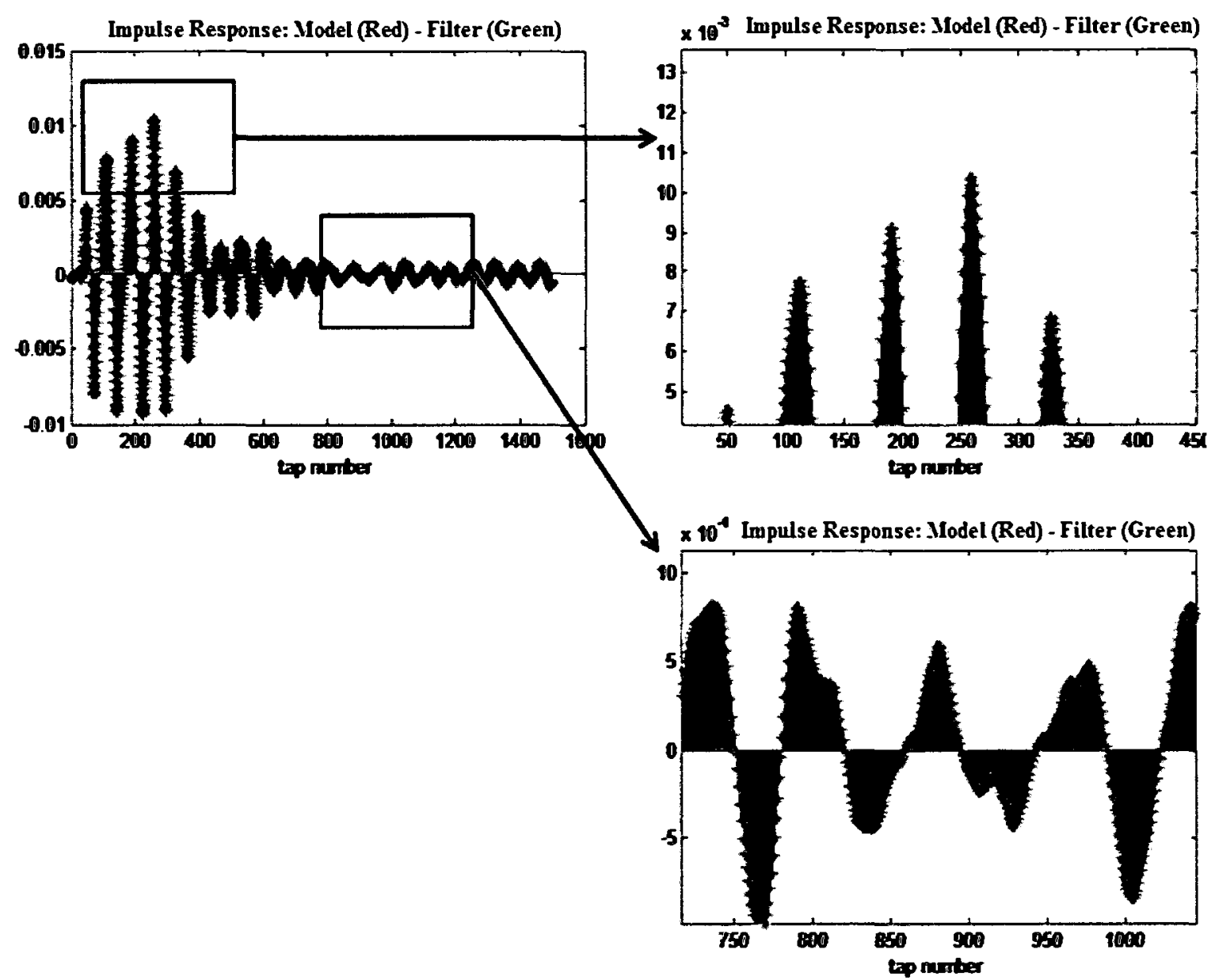

Figure 24 - Comparison of Adaptive Filtering Program Model Result with Adaptive Filtering Simulator Model Result.

The experiment was repeated for 50 trials and the data were averaged over all of the trials. The result was exactly the same as with a single trial and the models were thus equivalent (shown in Figure 25). 


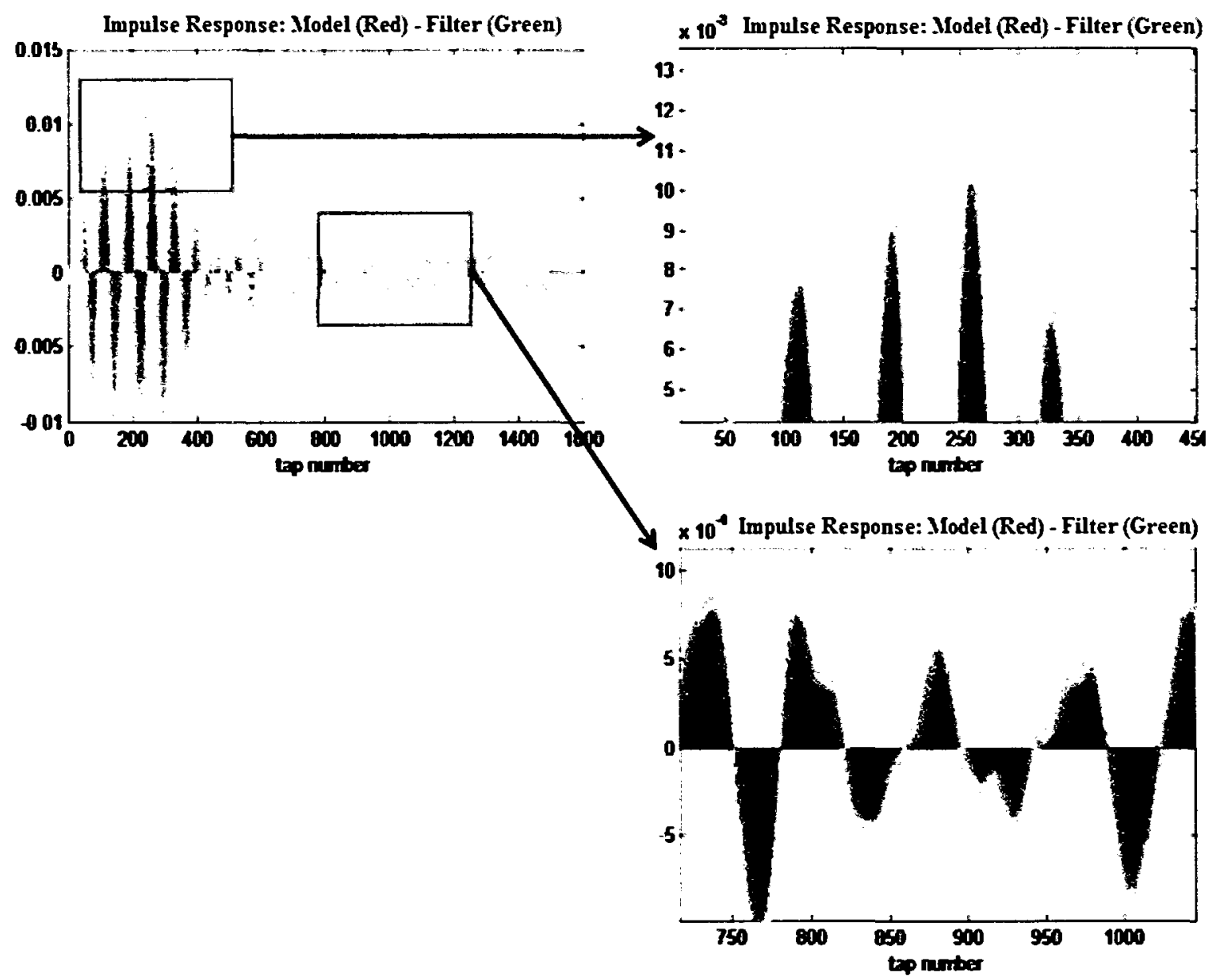

Figure 25 - Comparison of Instruments Algorithm Model Result with Adaptive Filtering Simulator Model Result over 50 Trials.

Also if the unknown system is assumed to be linear with a low contribution of non-linear components, a transfer function for the system could also be developed using classical Fourier Transform techniques. The model developed by the NLMS program or the adaptive filtering simulator should match a model developed using Fourier Transform techniques. The impulse response of a linear system using the Fast Fourier Transfer (FFT) can be defined from Equation 4 where $H(f)$ is the impulse response, $Y(f)$ is the output, and $X(f)$ is the input of the system.

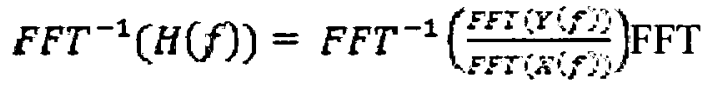


The result of this calculation was compared for a single trial and 50 trials with the model developed from the NLMS data processing algorithm of the medical instrument and the FFT calculations and are shown in Figure 26 and Figure 27.
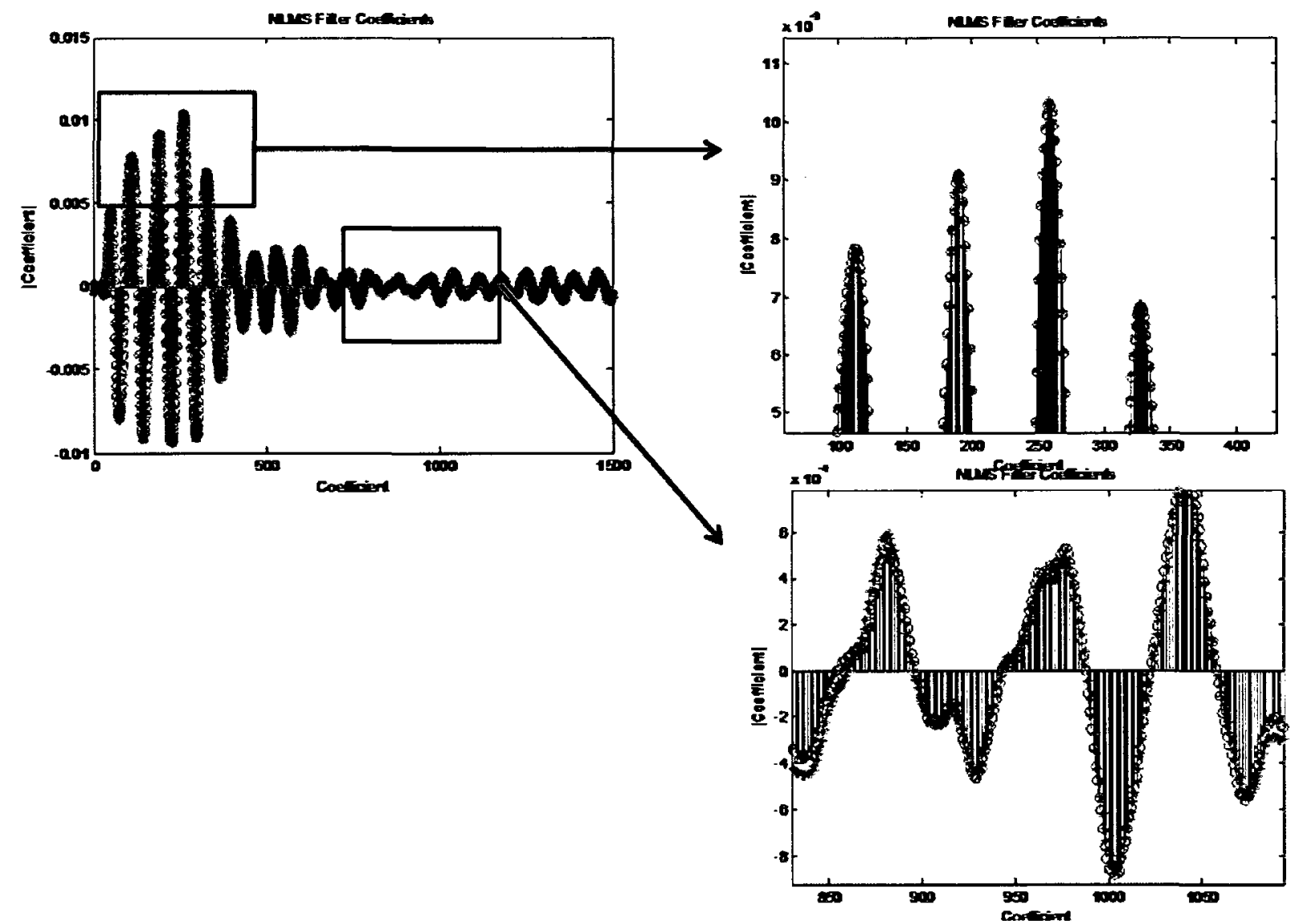

Figure 26 - Comparison of Instruments Algorithm Model Result with FFT Model Result.

The larger indexed coefficients of the medical instruments data processing algorithm did not compare exactly to those of the FFT Model Result. This is expected because the human chest in reality is not perfectly linear and ensemble averaging was not used to eliminate some of the non-linearities. This result shows that non-linearities are small and further investigation is shown in Chapter 7. The result improves when averaging over 50 trials shown in Figure 27. 

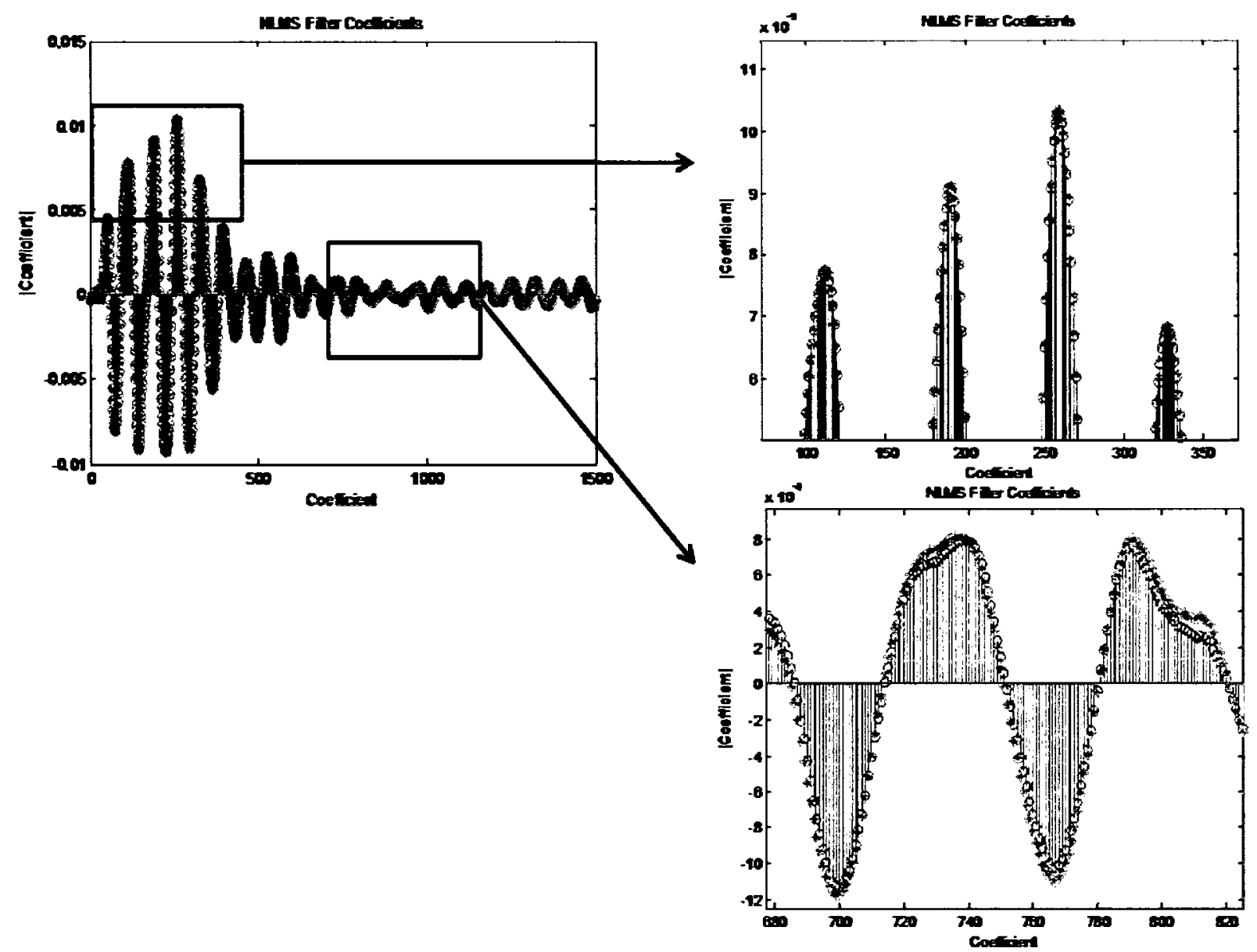

Figure 27 - Comparison of Instruments Algorithm Model Result with FFT Model Result over 50 Trials.

The results improve over 50 trials because many of the non-linearities in the system average out as they behave like random occurrences. As the number of trials increases, the results from the instrument's data processing algorithm and the FFT model will become increasingly similar because as with random sequences, if they are averaged infinite times, the result of the average would equal 0 . Therefore, the non-linearities within the system would not contribute to the system's transfer function. 


\subsection{Chapter Summary}

This chapter focused on simulations that were performed in order to select an appropriate input signal for the instrument, adaptive filter parameters, and test that the medical instruments data processing algorithm was implemented properly and stable. The input signal was selected to optimize the amount of information that could be recovered from the system transfer function. A set of 1500 coefficients and a step size $\mu$ of 0.296 were determined through experimentation to be appropriate values for the adaptive filtering algorithm. Finally, the program was tested against a well tested adaptive filtering simulator for proper implementation and also classical techniques including the Fourier Transform. The NLMS algorithm converged for all data that was measured and thus the algorithm and its configuration parameters were deemed appropriate and stable. 


\section{Chapter 5 - In Vitro Chest Phantom Experiments}

This chapter describes the development and results achieved from a series of physical chest phantom models. First for functional verification of the medical instrument's data processing algorithm implementation, a sound propagation model was developed to yield predictable results that could be verified using fundamental physics equations. Secondly, additional complexities were added to the base model in an effort to simulate sound propagation characteristics in actual human chests. Three chest phantom models were derived in total and described in this chapter namely the: open air column, plastic bucket, and chest phantoms.

\subsection{Open Air Column Phantom}

\subsubsection{Description}

The open air column phantom was constructed for verification of the adaptive filtering algorithm. The open air column phantom began with the attachment of a hollow foam cylindrical tube to the speaker/funnel components of the instrument. Each stethoscope was fastened using electrical tape at varying distances along the cylindrical tube. The open air column phantom is illustrated with dimensions in Figure 28. 


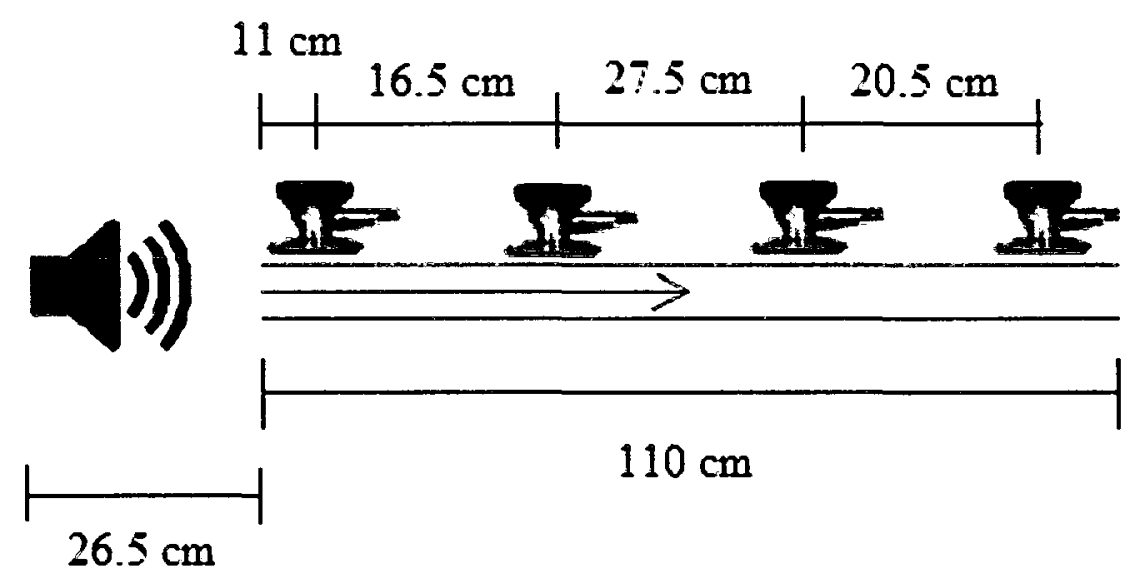

Figure 28 - Open Air Column Phantom

An experiment was performed to verify that the medical instruments data processing algorithm could calculate an accurate propagation delay between the stethoscopes by comparing the results to the physics of sound propagation $\left(t=\frac{d}{y} v=d / t\right)$ and using crosscorrelation. For this experiment, the delay of the input signal between the stethoscope closest to the speaker and all other stethoscope was calculated. The relative delay between each stethoscope and a reference stethoscope was calculated because it was difficult to determine the exact time offset even with the system calibration of the equipment for the input signal therefore making it impossible to compare the results of the NLMS and Cross-Correlation algorithms to theoretical predictions of the time delay of the input signal.

\subsubsection{Results}

The experiment involved the emission of a WGN pulse ranging from $0-4 \mathrm{KHz}$ with a duration of 5 seconds and acquiring it using each stethoscope. Results of the analysis compare the instruments data processing algorithm, physics of sound propagation, and cross-correlation in a delay calculation experiment for the open air column phantom. 


\begin{tabular}{|c|c|c|c|}
\hline $\begin{array}{c}\text { Stethoscope } \\
\text { Range }\end{array}$ & $\begin{array}{c}\text { Theoretical } \\
\text { Delay } \mathrm{t}=\mathrm{d} / \mathrm{v} \\
(\mathrm{ms})\end{array}$ & $\begin{array}{c}\text { NLMS Delay } \\
(\mathrm{ms})\end{array}$ & $\begin{array}{c}\text { Cross-Correlation } \\
\text { Delay (ms) }\end{array}$ \\
\hline $1-2$ & 0.6 & $1 \pm 0.2041$ & $1 \pm 0.2041$ \\
\hline $1-3$ & 1.34 & $1.25 \pm 0.4849$ & $1.25 \pm 0.4849$ \\
\hline $1-4$ & 1.808 & $2.875 \pm 0.7868$ & $2.875 \pm 0.7868$ \\
\hline
\end{tabular}

Table 5 - Experimental Results and their Standard Deviation that Compare Sound Propagation Delay Calculations between Three Algorithms.

The results in Table 5 show that the NLMS and Cross-Correlation algorithms calculate equal time delays between the stethoscopes and the reference stethoscope with equal standard errors because both methods calculate the maximum sound propagation delay path by calculating a maximum. The delay results compare reasonably to theoretical predictions. The NLMS and Cross-Correlation delays begin to deviate from theory as the sound propagation distance increases. Possible reasons for this include a theory with increased propagation distance, the number of reflections in the laboratory increase thus skewing results. Also, the experiments were not conducted in an acoustic chamber and many experiments in the laboratory conducted simultaneously throughout the duration of this project included machinery that emitted constant sound and thus were sources of noise. All of the delay calculation techniques yield delays within $+/-$ ms which is adequate to show that the instruments data processing algorithm provided correct results.

\subsubsection{Model Limitations}

The open air column phantom was only used for verification of the instruments data processing algorithm with known predictable sound models. There was no effort to 
simulate human chest characteristics and thus there were no model limitations. The instrument provided expected results when compared to theoretical predictions.

\subsection{Plastic Bucket Phantom}

\subsubsection{Description}

A phantom model was designed to simulate the behaviour of a real human chest. The first model was the Plastic Bucket Model built using the materials outlined in Table 6.

\begin{tabular}{|c|c|}
\hline Material & Quantity \\
\hline 5 Gallon Paint Bucket & 1 \\
\hline Camping Foam & 1 (sheet) \\
\hline Pipe Insulation & 1 (tube) \\
\hline
\end{tabular}

Table 6 - Materials used to build the Plastic Bucket Model

The purpose of the Plastic Bucket Model was to test the sensitivity of NLMS in detecting changes in delays as the volume of water in the bucket was varied. The goal of the model was to create different sound propagation paths to each stethoscope and observe changes in sound propagation delays. The input signal injected into the simulated trachea should arrive at the stethoscope in the array that has a field of view that is completely saturated with water as the speed of sound is much faster in water $(1500 \mathrm{~m} / \mathrm{s})$ versus that of air $(343 \mathrm{~m} / \mathrm{s})$. Also, if the water changes locations by rotating the bucket, the sound propagation path of the input signal to the other stethoscopes in the array would change and a decrease in delay would be observed leading to the ability of the array to detect changes in location of the water. 
The procedure for building the Plastic Bucket Model is as follows. The camping foam was rolled and inserted into the paint bucket. A Y-Shaped pipe was constructed in order to simulate the trachea using the pipe insulation. The simulated trachea was inserted into the camping foam (as close as possible to center). Finally the stethoscope array was attached to the paint bucket. An illustration is shown in Figure 29 .

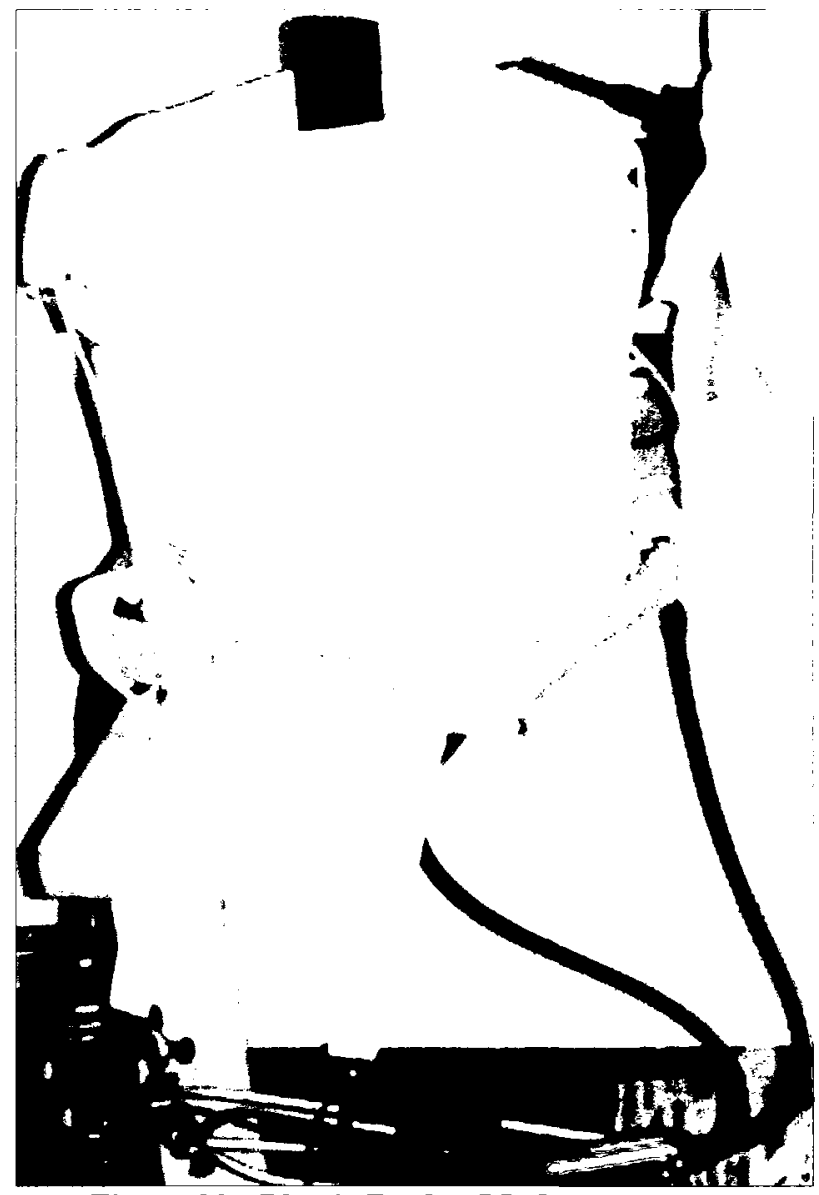

Figure 29 - Plastic Bucket Model Apparatus

An experiment was performed using the Plastic Bucket Model where the bucket was filled with one litre of water. It was then tilted to one side such that two of the four stethoscopes attached around the bucket had water within their field of view of the sound propagation path. The WGN input signal ranging from $0-4 \mathrm{KHz}$ was emitted down the 
simulated trachea and into the bucket to be measured by the stethoscope array. The delay was calculated between each stethoscope and the input signal using the NLMS algorithm.

\subsubsection{Results}

The delay calculations showed a reduction in delay by the input signal and the two stethoscopes that had the presence of water within their field of view. The other two stethoscopes reported constant increased delays.

\subsubsection{Model Limitations}

Due to a poor interface between the stethoscope heads and the plastic surface of the bucket, the above experimental results were not repeatable and thus abandoned. At times, very erroneous results were detected and thus there was a need to develop a better phantom focusing on emulating an improved skin to stethoscope head interface. This led to the birth of the Chest phantom that aimed at improving the stethoscope head to phantom interface.

\subsection{Chest Phantom}

\subsubsection{Description}

A chest phantom is an apparatus designed to simulate the behaviour of a real human chest. In this case, a phantom model of a human chest was designed and built in order to perform test cases for the NLMS algorithm and stethoscope array concepts. The model was constructed using camping foam, pipe insulation, a tire inner tube, clear tubing, a syringe, and hot glue. The camping foam was rolled to form a cylinder with a diameter of 0.12 meters and a length of 0.52 meters. The pipe insulation was cut to form two tubes with a diameter and length of 0.04 meters and 0.3 meters respectively. The tubes were 
inserted into one end of the camping foam at equidistance's from the edge of the camping foam at approximately 0.05 meters. A Y-Pipe was constructed measuring 0.2 meters in length using the remaining pipe insulation and attached to the ends of the tubes. After removing the valve from the tire inner tube stem, the tire tube with an inner diameter of $0.15 \mathrm{~m}$ was slipped over the camping foam cylinder 0.3 meters from the top edge. A 1.8 meter clear piece of tubing with a syringe inserted at one end was attached to the tire stem. Figure 30 illustrates the chest phantom model and a block diagram attached to the sound acquisition apparatus. The stethoscope harness attaches around the tire inner tube. This point is regarded as the chest surface.
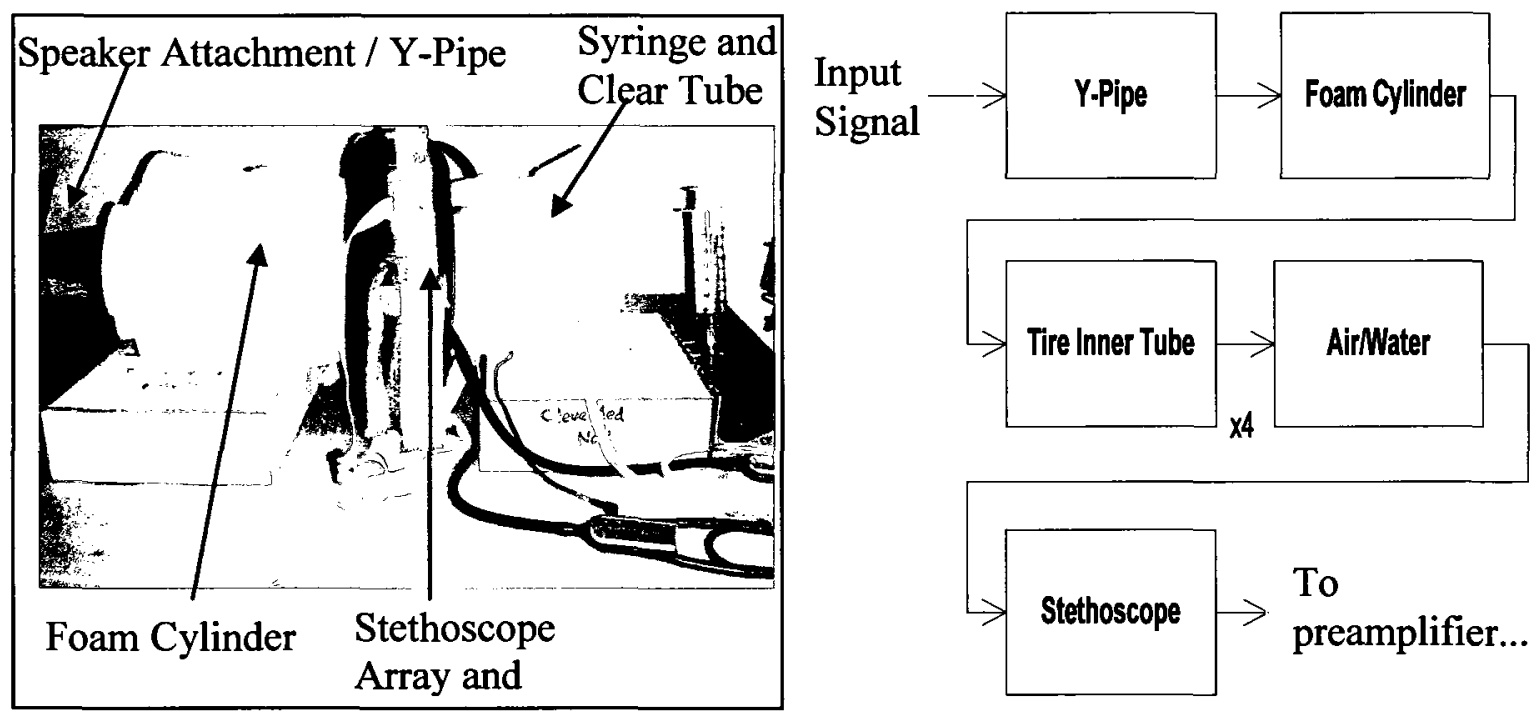

Figure 30 - Chest Phantom (Unknown System) with the Stethoscope Array and Harness Attached to the Tire Inner Tube or Simulated Chest Surface

Validation of the system was performed by injecting known volumes of water into the tire inner tube and running the system by playing the input signal using the subwoofer and measuring it with the stethoscope array. Volumes of water started from $0 \mathrm{cc}$ to $40 \mathrm{cc}$ with an increment of $5 \mathrm{cc}$ per trial and each trial involved 195 individual 5 second measurement of the input sound signal in order to carry out ensemble averaging which is 
typical for the NLMS algorithm.

Due to gravity, water injected into the tire inner tube flowed to the bottom as seen in Figure 30. The speed of sound in water $(1500 \mathrm{~m} / \mathrm{s})$ is much faster than in air $(343 \mathrm{~m} / \mathrm{s})$ and as the volume of water increases in the tube, the lower stethoscope (Figure 31) will detect the input signal faster than the other stethoscopes due to a shortened sound propagation delay.

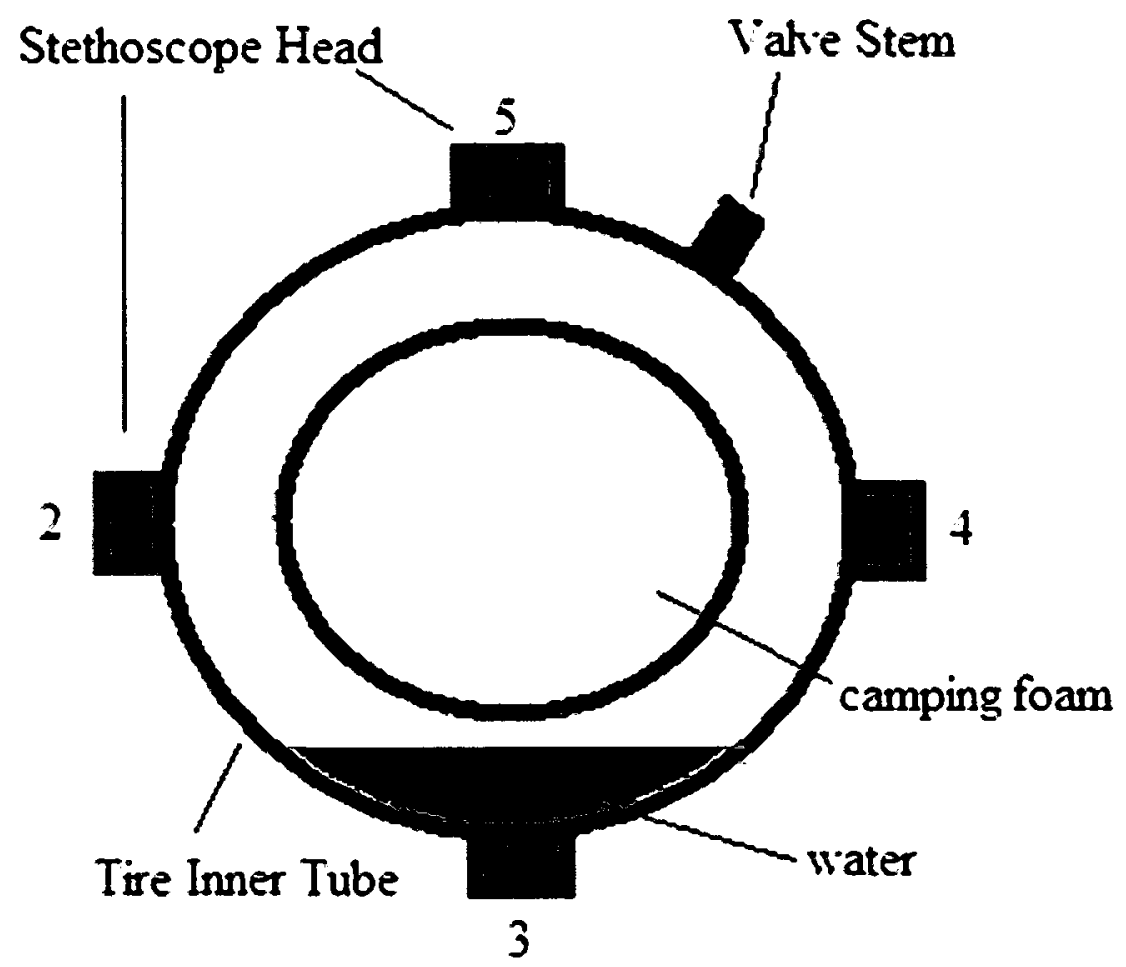

Figure 31 - Illustration of the Stethoscope Array and Inner Tire Tube Assembly. Water Injected into the Tube Flows to the Bottom due to Gravity.

The propagation delay of the input signal was measured using NLMS adaptive filtering by looking at the coefficient values of the model. The system transfer function was also investigated to search for changes in the frequency spectrum that could be useful in metric determination such as frequency peaks or changes in the area under the curve. 


\subsubsection{Results}

The expected result was that the propagation delay of the input signal to the stethoscopes connected to channels 2,4 , and 5 of the pre-amplifier would remain at a constant value and not necessarily the same value due to their varying locations. However, the propagation delay of the input signal to the stethoscope attached to channel 3 of the preamplifier would decrease as the volume of water that gathers at the bottom of the tire inner tube increased. This is because the speed of sound in water $(1500 \mathrm{~m} / \mathrm{s})$ is approximately 5 times faster than that in the air $(343 \mathrm{~m} / \mathrm{s})$. Figure 32 shows the propagation delay for the stethoscope attached to channel 2 of the pre-amplifier as being constant. This behaviour is also reflected for stethoscopes 4 and 5 .

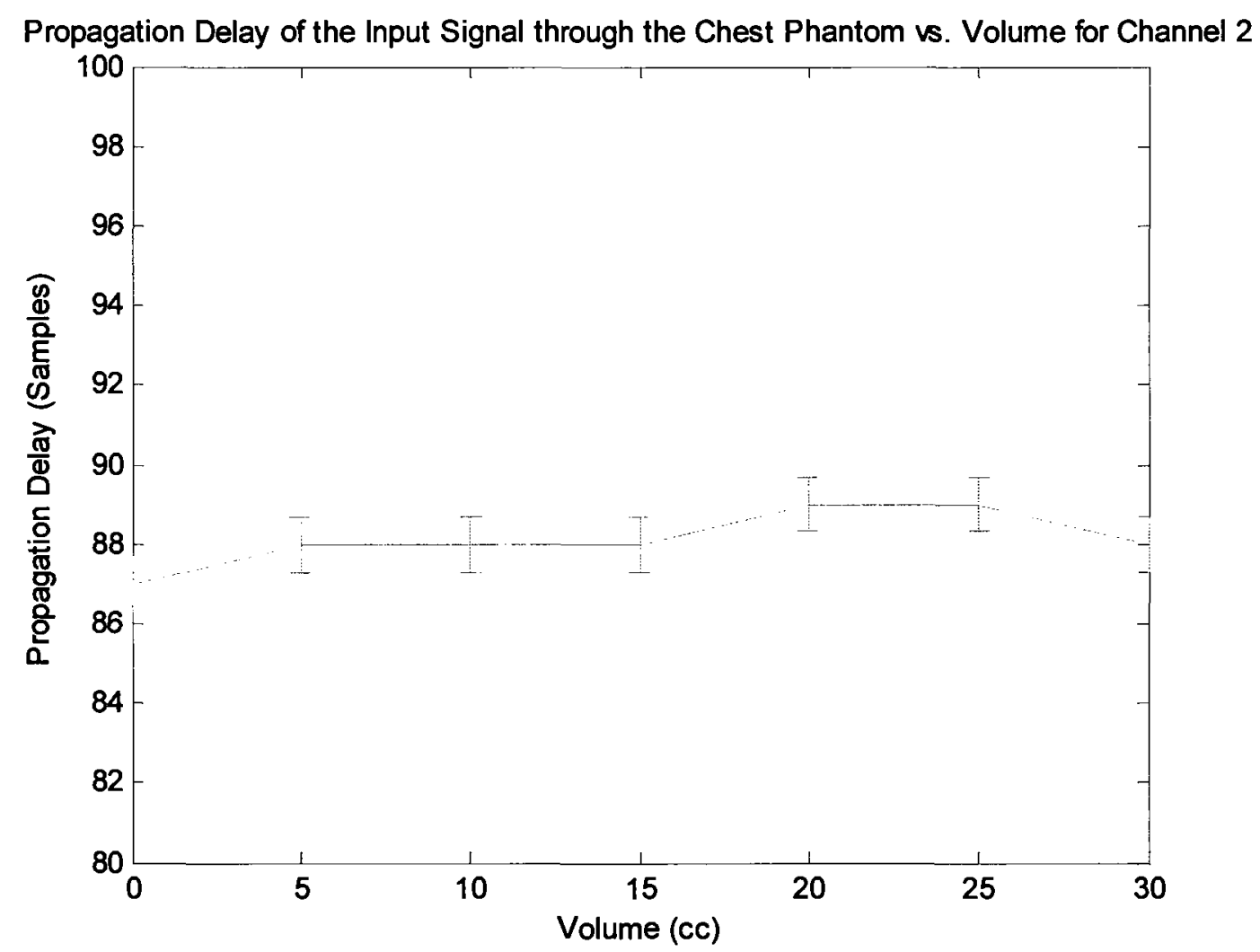

Figure 32 - Propagation Delay for a Stethoscope Without the Presence of Water Within its Field of View 
Figure 33 shows that as the volume of water in the inner tube increases, the propagation delay of the input signal through the chest phantom model to the lower stethoscope decreases similar to hydraulic conductivity shown by (Canter, Knox, \& Fairchild, 1988). This makes intuitive sense because eventually the lower portion of the tire inner tube will saturate with water and the propagation delay will remain constant.

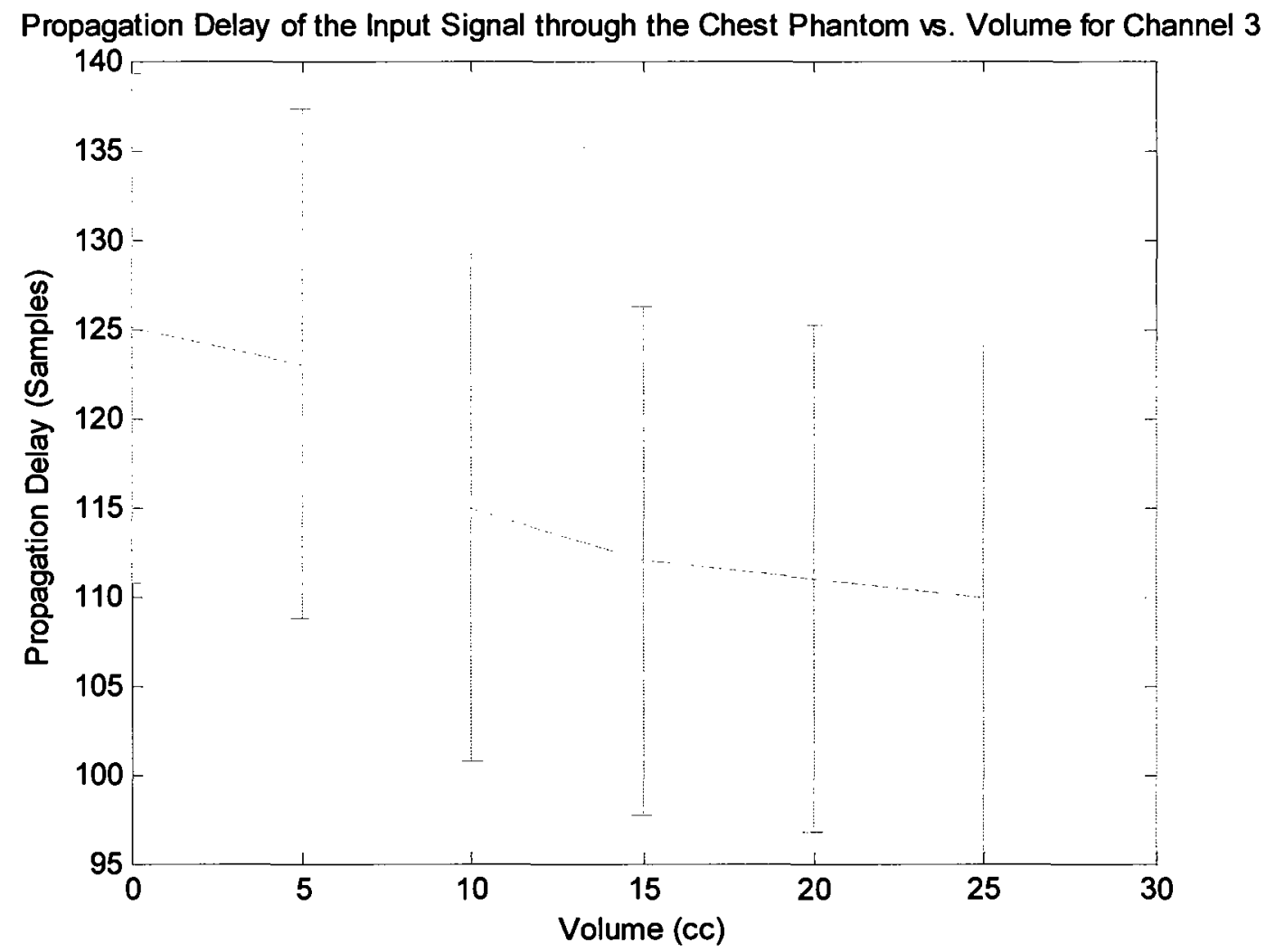

Figure 33 - Propagation Delay for a Stethoscope with the Presence of Water Within its Field of View It was observed that with no water volume in the chest phantom that the transfer function behaved like a low-pass filter with no DC component. Furthermore, there were two evident frequency spikes at $117.2 \mathrm{~Hz}$ and $242.2 \mathrm{~Hz}$. As the volume of injected water into the chest phantom increased, the frequency spikes became sharper. This is illustrated in Figure 34 with a plot showing 3 transfer functions obtained with different volumes of water within the chest phantom model. 
The next step was to try the instrument on healthy humans to determine if similar behavior to the chest phantom model could be observed.

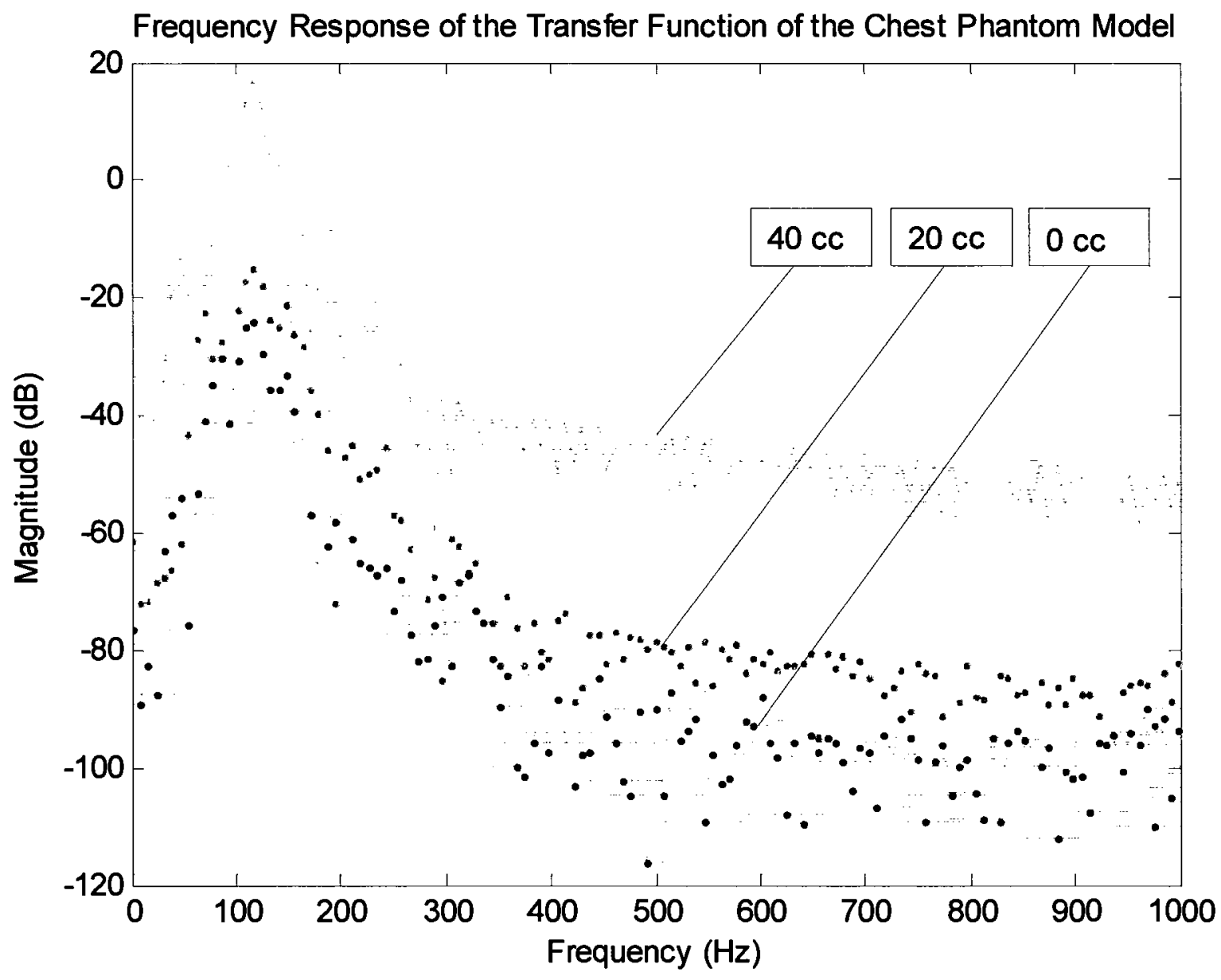

Figure 34 - Above is the magnitude response of the Transfer Function of the Chest Phantom Model with No Volume, 20cc, and 40cc of Water.

\subsubsection{Model Limitations}

The transfer functions shown in Figure 34 are similar to transfer functions obtained through human trials conducted by (Ciftci, Yeginer, Sen, Cini, \& Kahya, 2004) in which two frequency peaks were also detected in the same frequency range. 
By using a tire inner tube, the Chest phantom models the lungs as a large volume rather than using the concept of the alveoli as a group of sacks. The consequences of this will have to be investigated as the chest phantom model becomes more complex to simulate actual chest behaviour. A foam surface should also be inserted between the stethoscopes and the tire inner tube to simulate the skin on the chest. This would cause further attenuation of the input sound signal and will likely show in the system transfer function as a more defined low-pass filtering effect.

\subsection{Chapter Summary}

This chapter described a series of in vitro chest phantom experiments and the results of these experiments. The chapter also described the performance of system verification experiments. The incremental development process of a simple model into a complex model was also shown. Finally, limitations to using these models were discussed. 


\section{Chapter 6 - In Vivo Experiments}

In this chapter, experiments were performed on human participants in order to: 1) test the validity of the chest phantom model, 2) determine if the medical instrument could provide reasonable sound propagation delay estimates and 3) if the sound propagation delay and system transfer function changed with posture variations.

\subsection{Experimental Protocol}

An experiment was performed to compare transfer functions obtained by the chest phantom model and those obtained from human testing. Tests were performed on 3 healthy male subjects between the ages of $20-24$ positioned in the sitting, lying on their: back, left side, right side, and flat on stomach postures in accordance with the Health Research Ethics Board protocol. The participants were asked to breathe normally at tidal volume as an input signal of WGN from $0-4 \mathrm{kHz}$ was injected into their mouths and recorded on their chest surface. The collected data were run through the NLMS algorithm over a number of iterations depending on the experiment. The model's coefficients upon convergence of the adaptive filtering algorithm were used to calculate the impulse response of the system. The impulse response of the system was calculated for a range of frequencies between 0 and $\pi$ rads/sample and then plotted to reveal the systems transfer function. 


\subsection{In Vivo Results - Patient Sitting}

The results obtained for each human participant of the experiment were ensemble averaged over 195 trials. Propagation delays in the same range as those measured in the chest phantom model were detected for each participant. These reasons show a main peak at approximately $85 \mathrm{~Hz}$ and a suggests what appears to be a smaller side lobe at $170 \mathrm{~Hz}$. A peak detector that looked for a zero slope from the systems transfer function found two peaks at $86.0 \pm 2.5 \mathrm{~Hz}$ and $172.0 \pm 2.5 \mathrm{~Hz}$. It was expected that the frequency peaks between the chest phantom model and the human body would differ due to the material and geometrical differences between the two systems. The resulting transfer functions for each participant were constant as the subjects were all healthy and are displayed in Figure 35. 


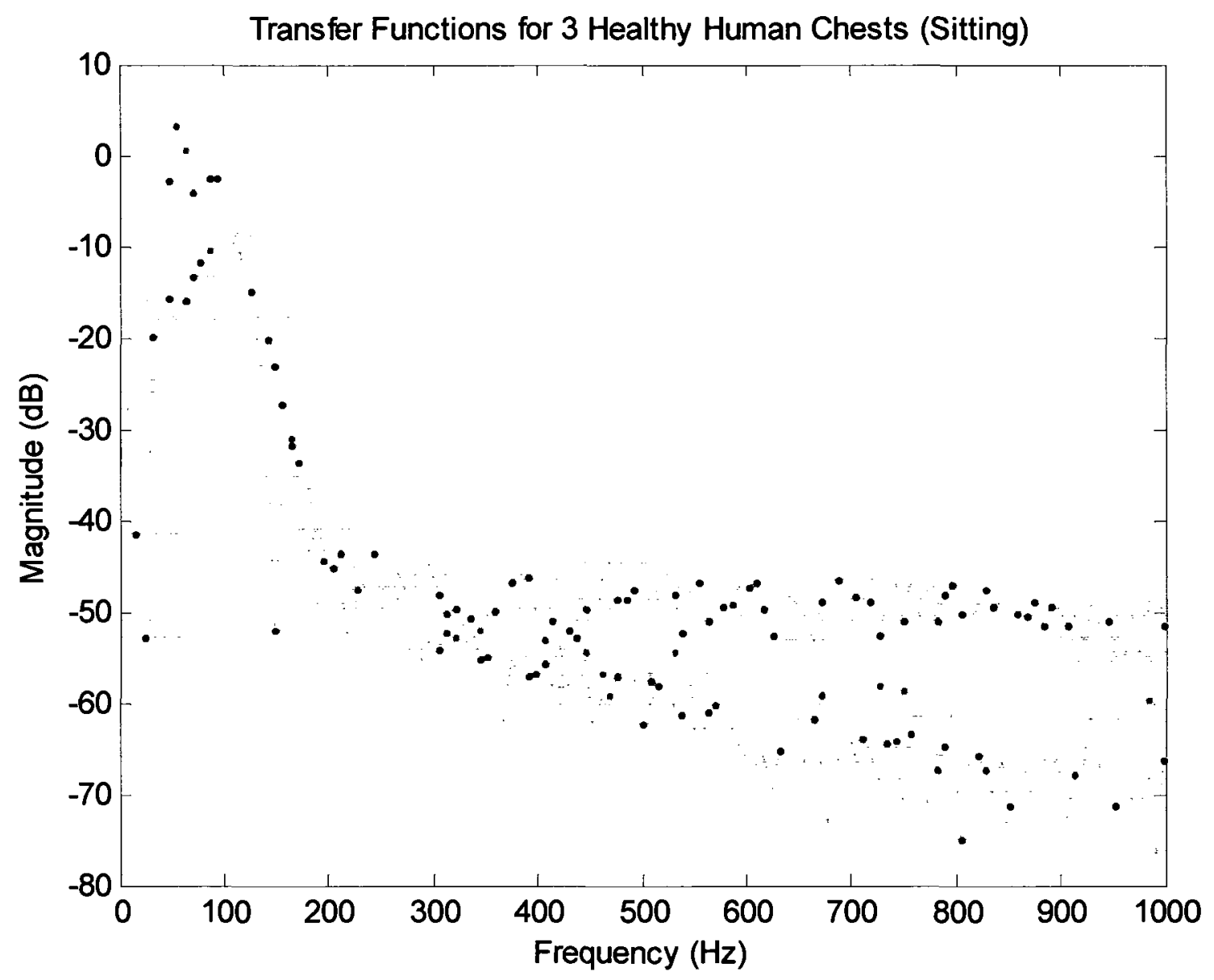

Figure 35 - Transfer Functions for 3 Healthy Human Chests (Sitting Upright).

\subsection{In Vivo Results - Patient Lying on Back}

The results obtained for each human participant of the experiment were ensemble averaged over 15 trials. Propagation delays in the same range as those measured in the chest phantom model were also detected for each participant. Two frequency peaks were observed around the same frequency locations $86.0 \pm 45.6 \mathrm{~Hz}$ and $172.0 \pm 45.6 \mathrm{~Hz}$ as in the sitting posture. The peaks shown in Figure 36 have a much larger magnitude than those of the sitting posture experiment. This is likely due to the fact that when the participants were lying down, their lungs were more compressed than in the sitting upright posture 
and thus the volume of air was decreased causing the inner lung tissue surfaces to become closer together. This caused the lungs to behave like a channel of water as tissue is primarily water. As was observed in the chest phantom experiments, as the volume of water inside of the phantom increased, the two frequency peaks became more prominent.

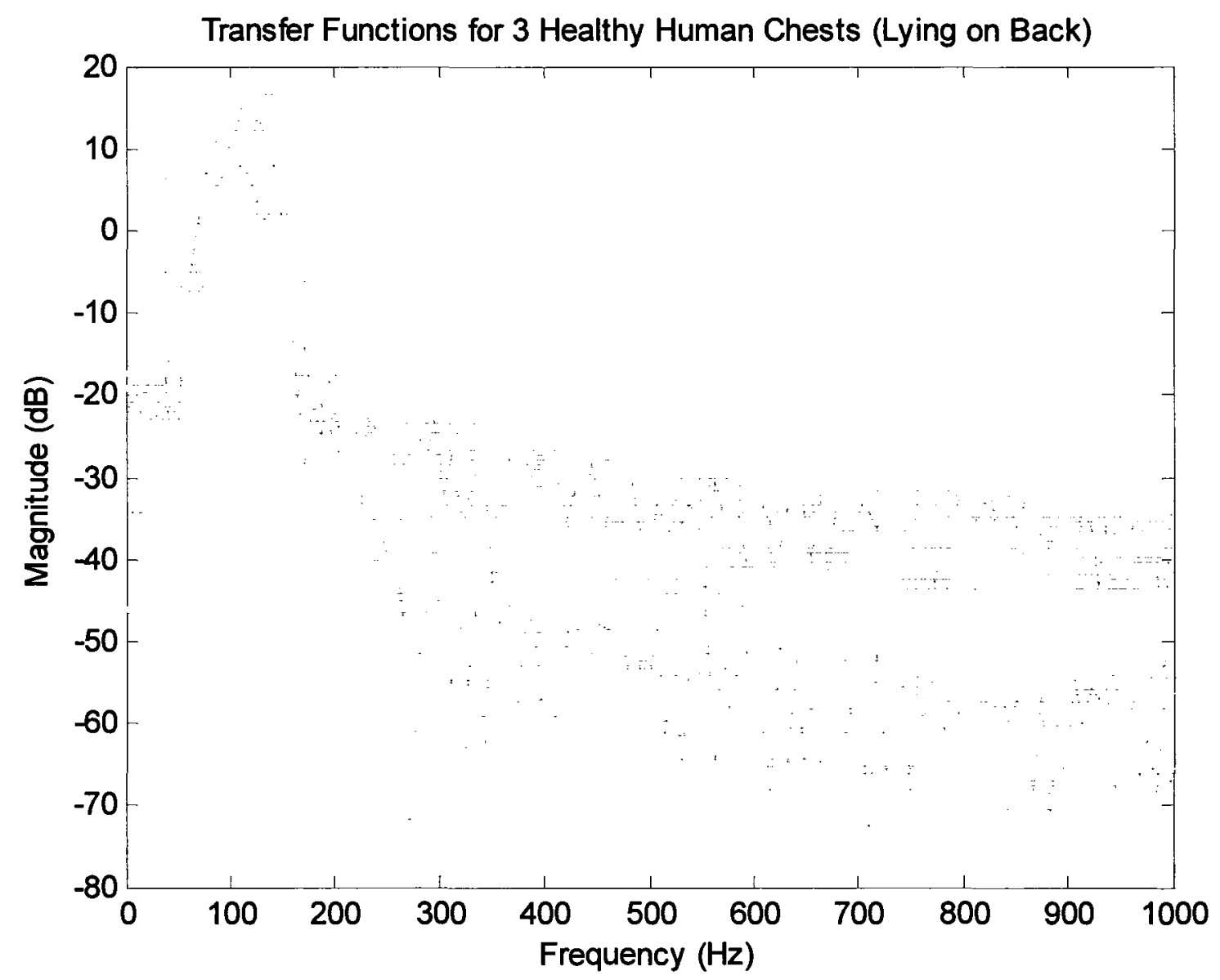

Figure 36 - Transfer Functions for 3 Healthy Human Chests (Lying on Back).

\subsection{In Vivo Results - Lying on Right Side}

In this experiment it was expected that the two stethoscopes placed over the right lung would detect decreased propagation delays as opposed to the other two stethoscopes placed over the lung. Similar to the reason that the propagation delay decreased when participants were lying flat on their backs, the right lung in this case should be more 
compressed and behave more like a sound propagation channel filled with water due to an increased amount of tissue and a decreased amount of air within the right lung. After collecting results from each human participant of the experiment over 15 trials, for 2 out of the 3 participants, a decrease in propagation delay was observed in the stethoscope over the right lung when comparing two stethoscopes placed over opposing lungs. Also, the transfer function for the stethoscope placed over the right lung in Figure 38 showed an increase in magnitude at the two peak frequencies as published by (Mulligan, Adler, \& Goubran, Detecting Regional Lung Properties using the Audio Transfer Function of the Respiratory System, 2009) when compared to Figure 37. The opposite would be expected for participants lying on their left side in the next experiment. 


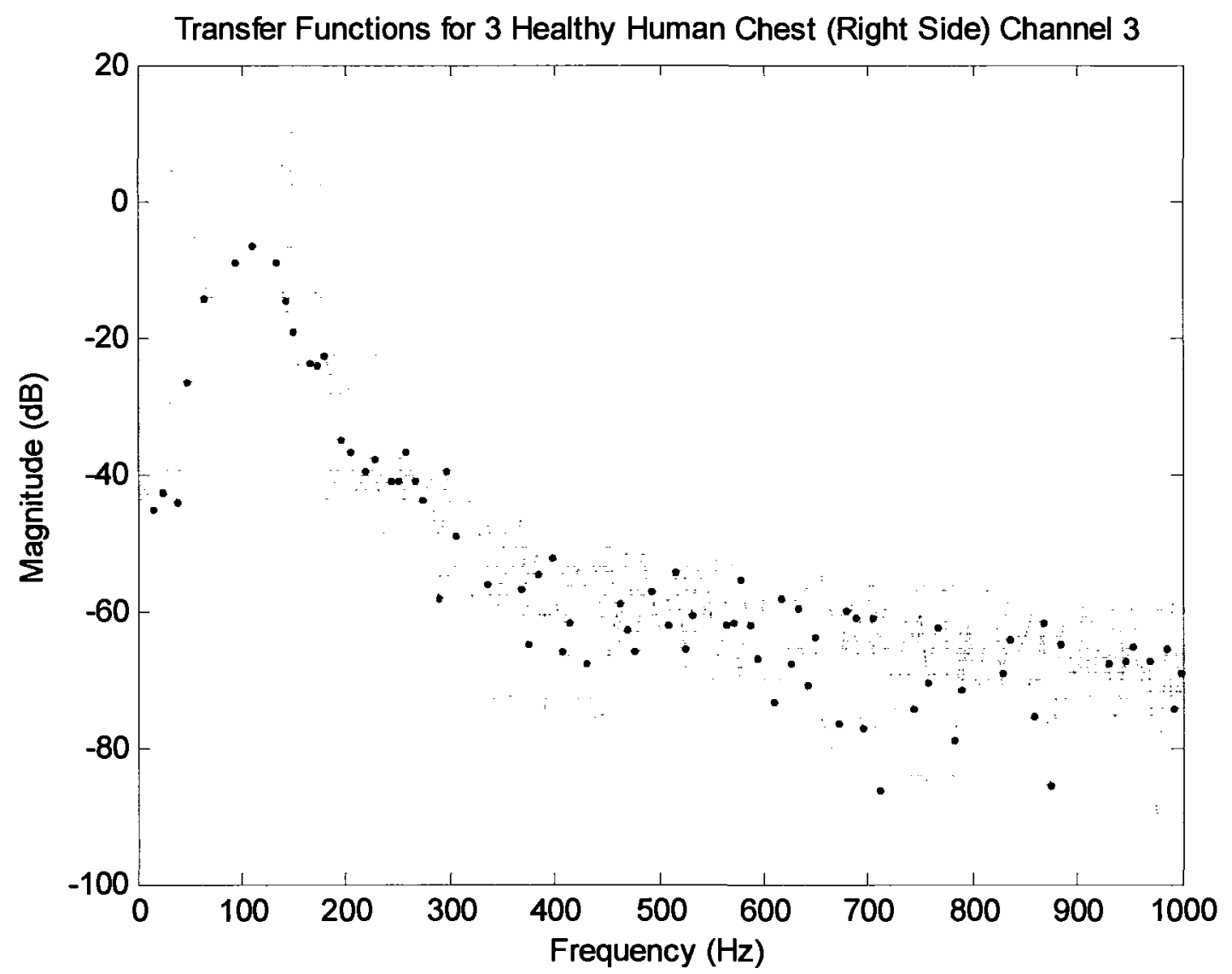

Figure 37 - Transfer Function for 3 Healthy Human Chests of Stethoscope 2 (Channel 3) Placed over their Left Lung. Participants are Lying on their Right Side. 


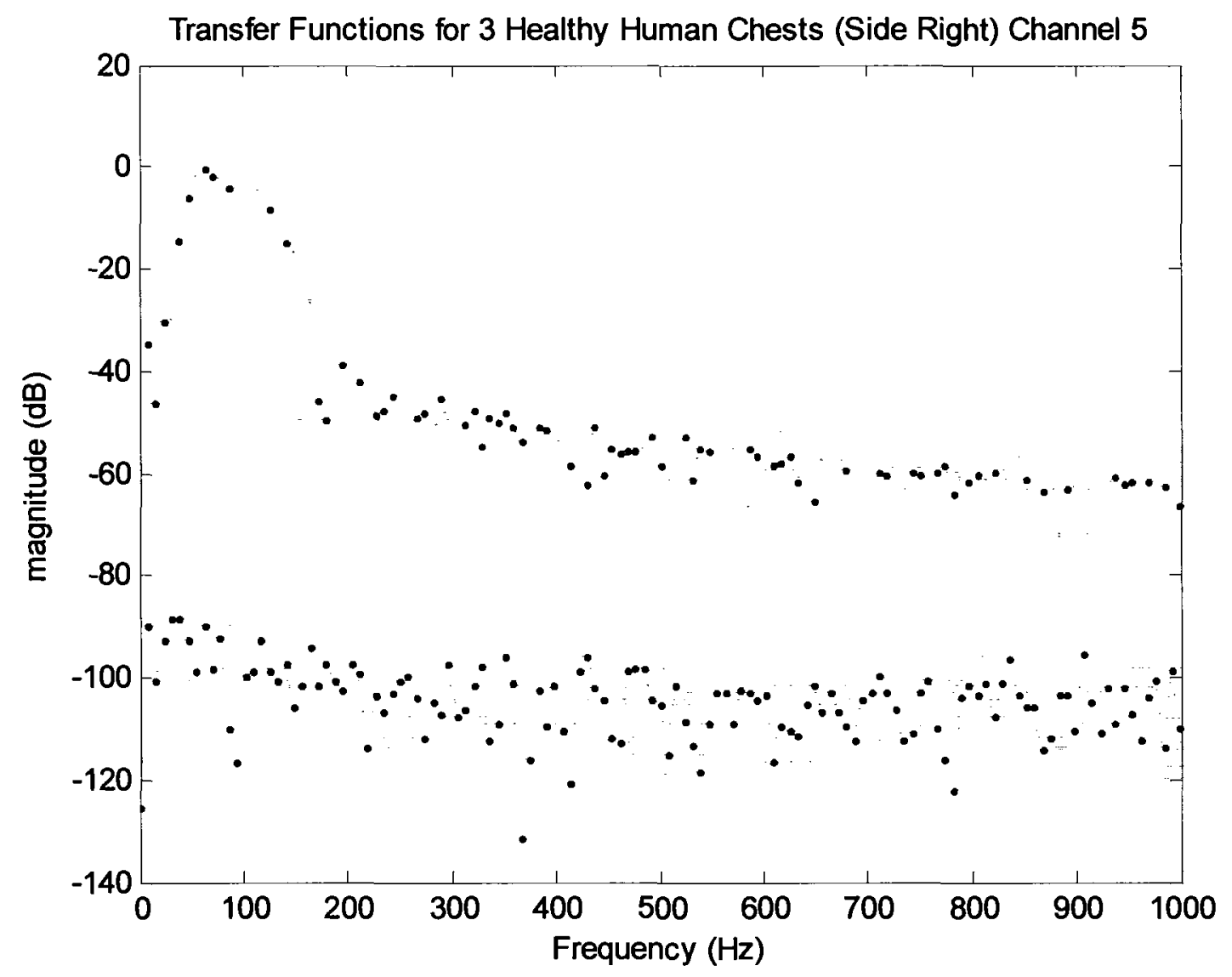

Figure 38 - Transfer Function for 3 Healthy Human Chests of Stethoscope 4 (Channel 5) Placed over their Right Lung. Participants are Lying on their Right Side.

\subsection{In Vivo Results - Lying on Left Side}

This experiment was performed with the exact same procedure as the in section 6.4 however, participants were asked to lie on their left side. The opposite effect was expected to be observed in this experiment. When the magnitudes of the transfer functions in Figure 39 and Figure 40 at the two peak frequencies were compared, the magnitudes at the peaks for the stethoscope placed over the left lung were larger for all 3 participants. This is exactly what would be expected. 


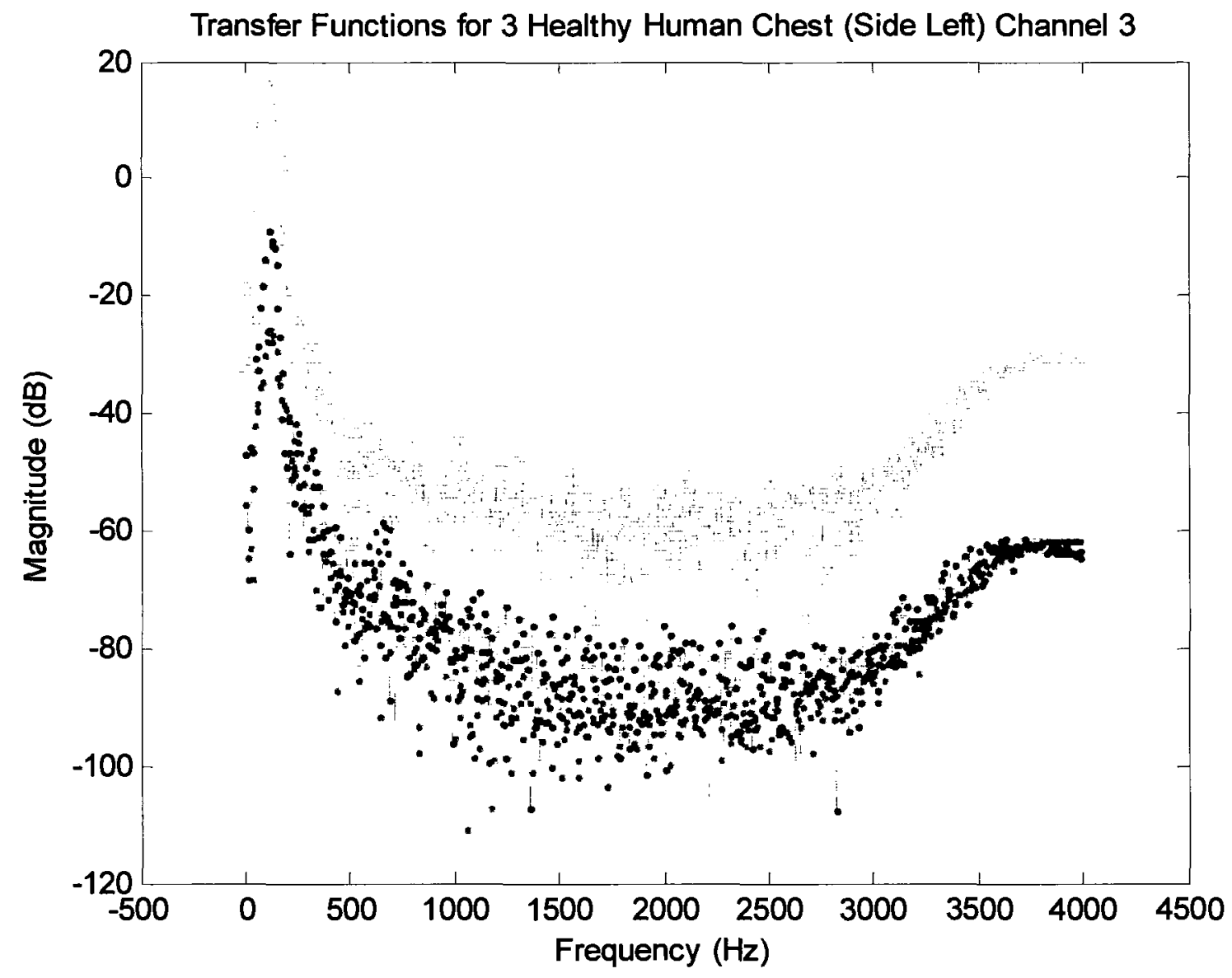

Figure 39 - Transfer Function for 3 Healthy Human Chests of Stethoscope 2 (Channel 3) Placed over their Left Lung. Participants are Lying on their Left Side. 


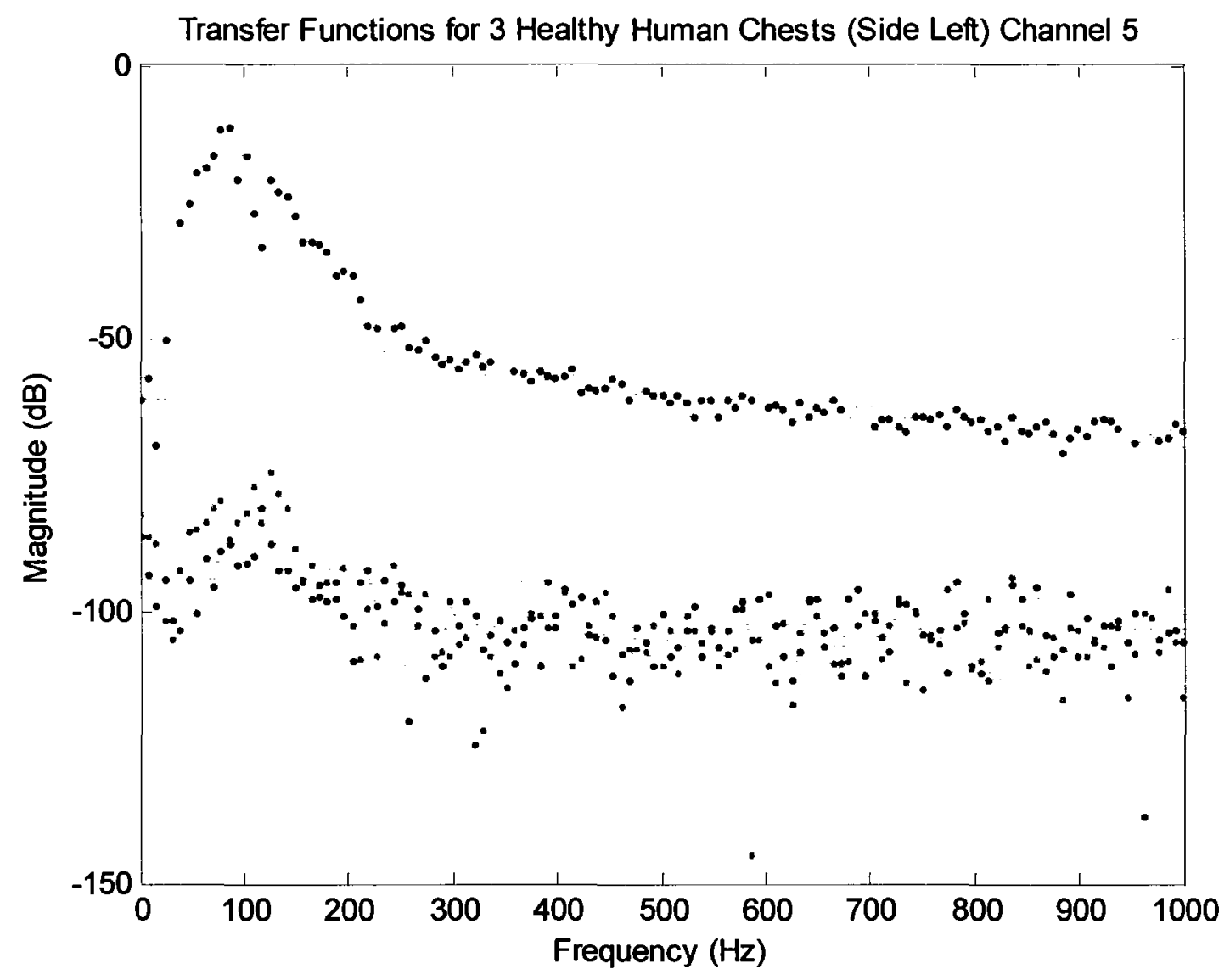

Figure 40 - Transfer Function for 3 Healthy Human Chests of Stethoscope 4 (Channel 5) Placed over their Left Lung. Participants are Lying on their Left Side.

\subsection{In Vivo Results - Flat on Stomach}

The results obtained for this experiment were expected to correlate with those in section 6.3 as both postures position the lungs in similar forms. Results for 1 participant were equivalent as this participant was lean and contained similar amounts of body fat on the anterior and posterior chest areas. The other two participants contained a higher percentage of body mass on the anterior chest area and thus an increase in propagation delay for the anterior stethoscopes was expected. This is observed in the transfer function plotted in Figure 41. For 2 of the 3 participants there was a decrease in the two peak 
frequency magnitudes signifying an increase in sound propagation delay.

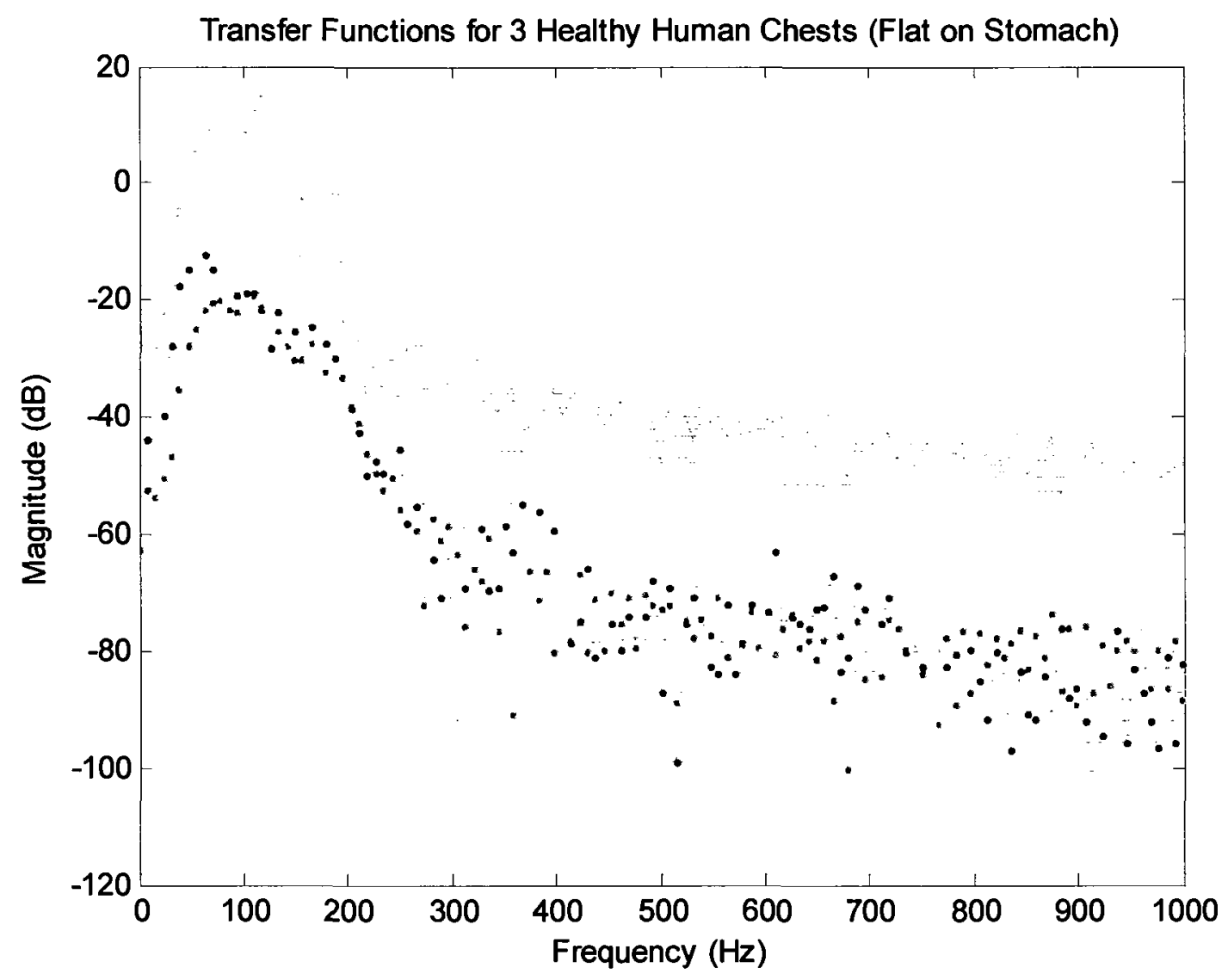

Figure 41 - Transfer Function for 3 Healthy Human Chests. Participants are lying flat on their stomachs.

\subsection{In Vivo Results - All Stethoscopes}

In this section, two types of frequency responses were plotted: 1) the frequency responses for all postures on one plot for each participant, and 2) the frequency responses for all stethoscopes on one plot for one participant. For the frequency responses of all postures on one plot, each participant generally when lying flat on their backs, yielded the lowest sound propagation delay with a corresponding transfer function curve showing the highest magnitude as shown in Figure 42, Figure 43, and Figure 44. In the frequency 
responses for all stethoscopes on one plot for one participant, the transfer function curve labelled 1 corresponded to the curve where the lowest propagation delay would be expected for the corresponding posture. It is shown in the frequency and impulse responses of Figure 46, Figure 47, Figure 48, and Figure 49 that the instrument is capable of detecting the expected lowest sound propagation delay path for a corresponding posture.

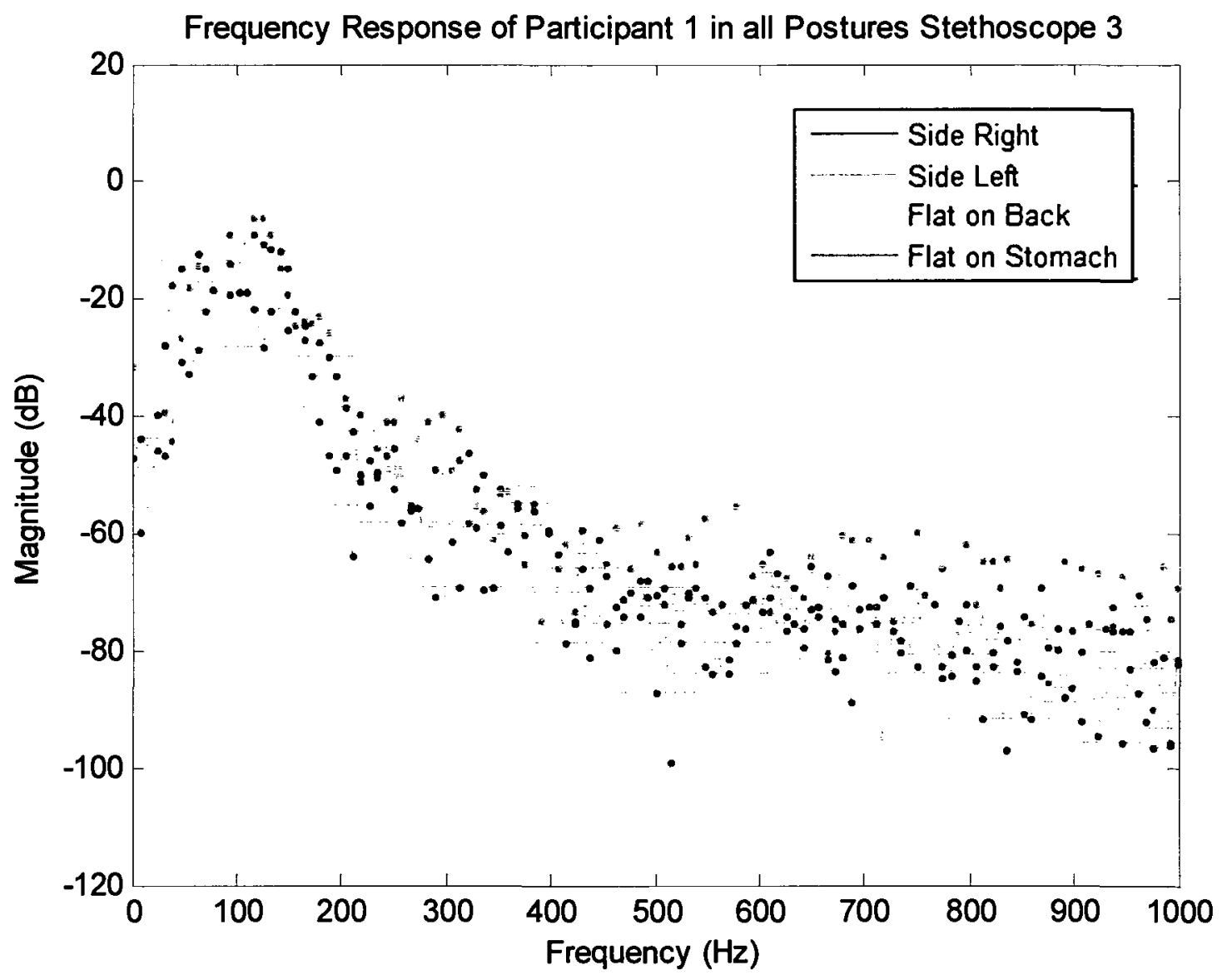

Figure 42 - Frequency Responses of Participant 1 in all Postures Plotted Together. 


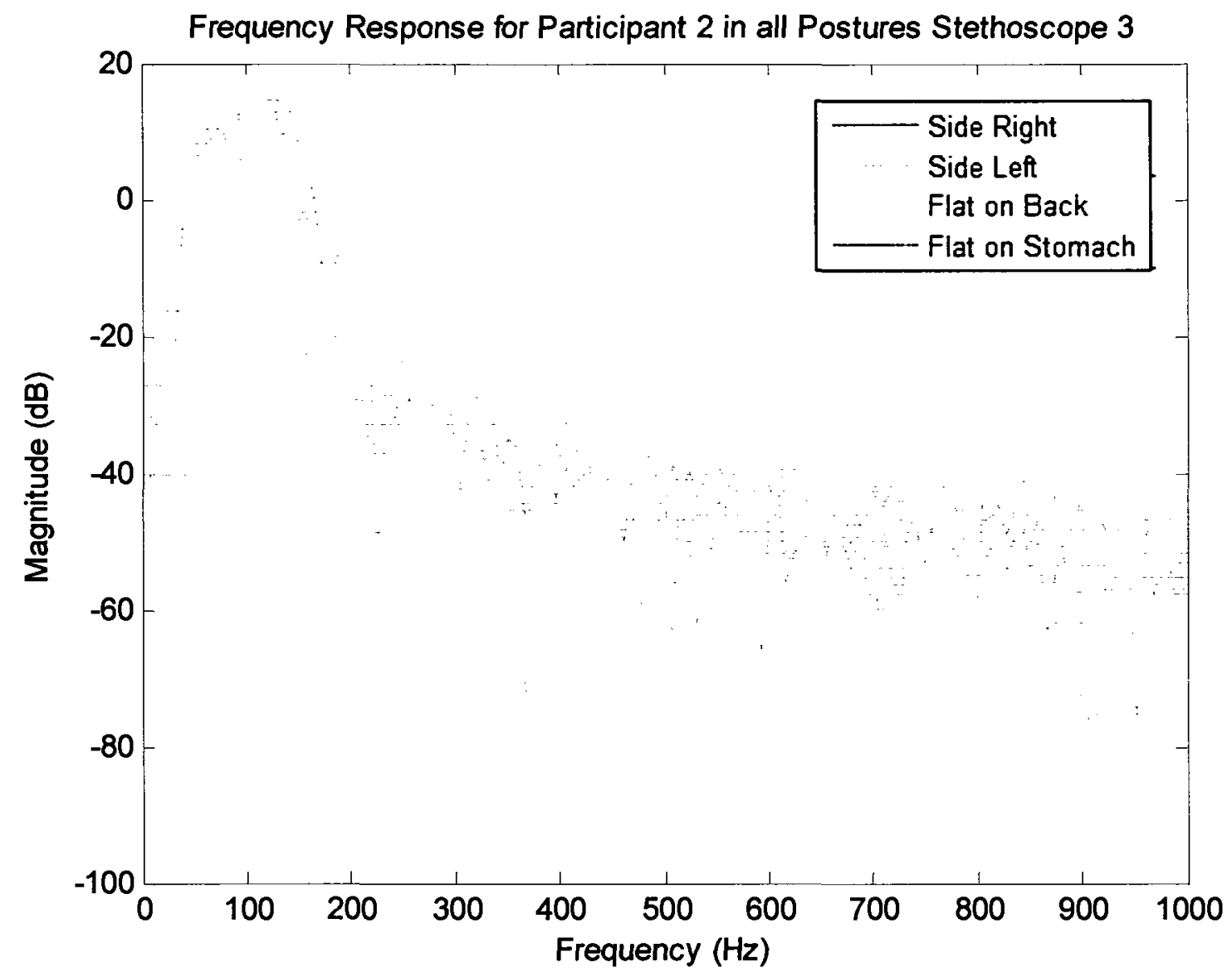

Figure 43 - Frequency Responses of Participant 2 in all Postures Plotted Together. 


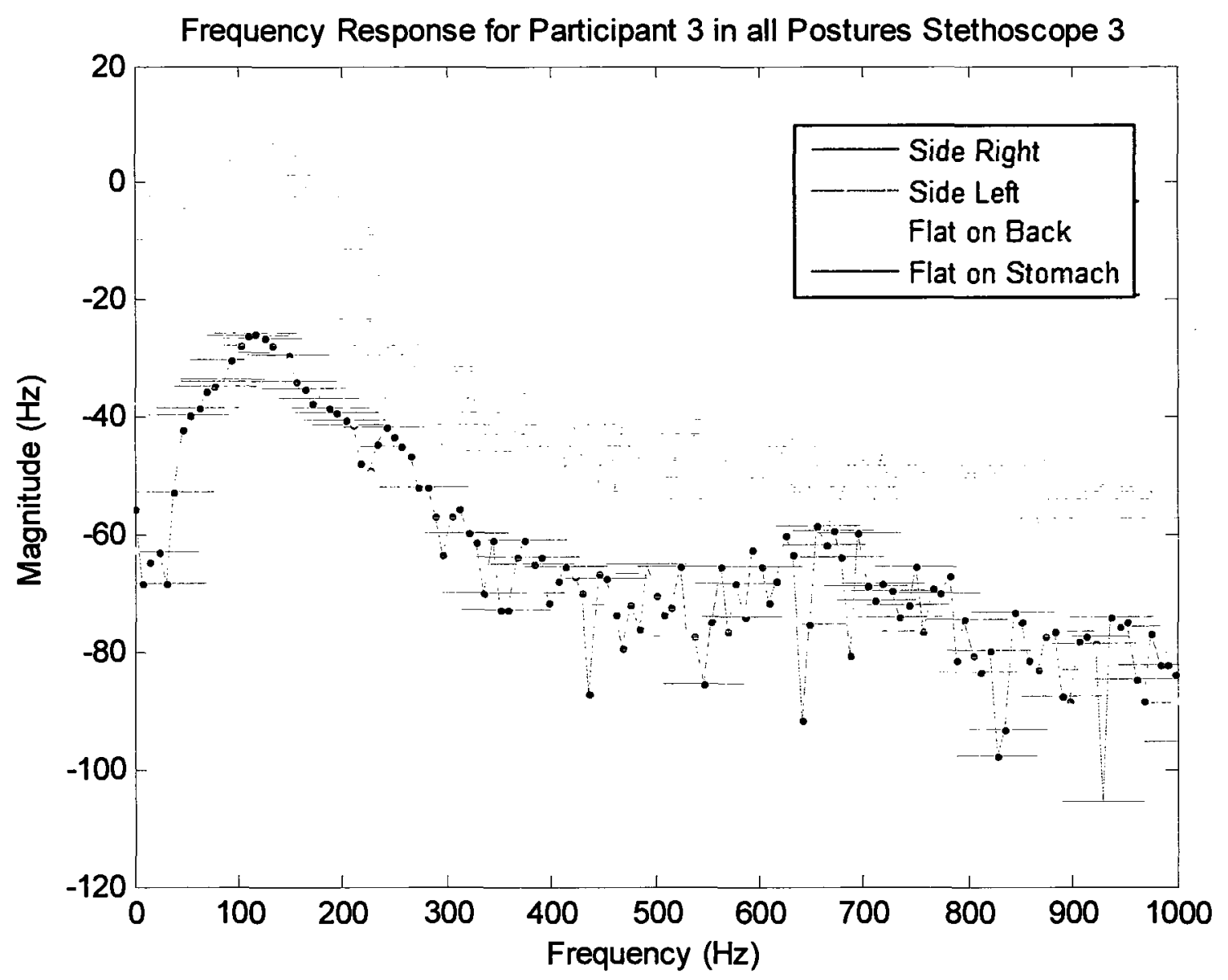

Figure 44 - Frequency Responses of Participant 3 in all Postures Plotted Together. 

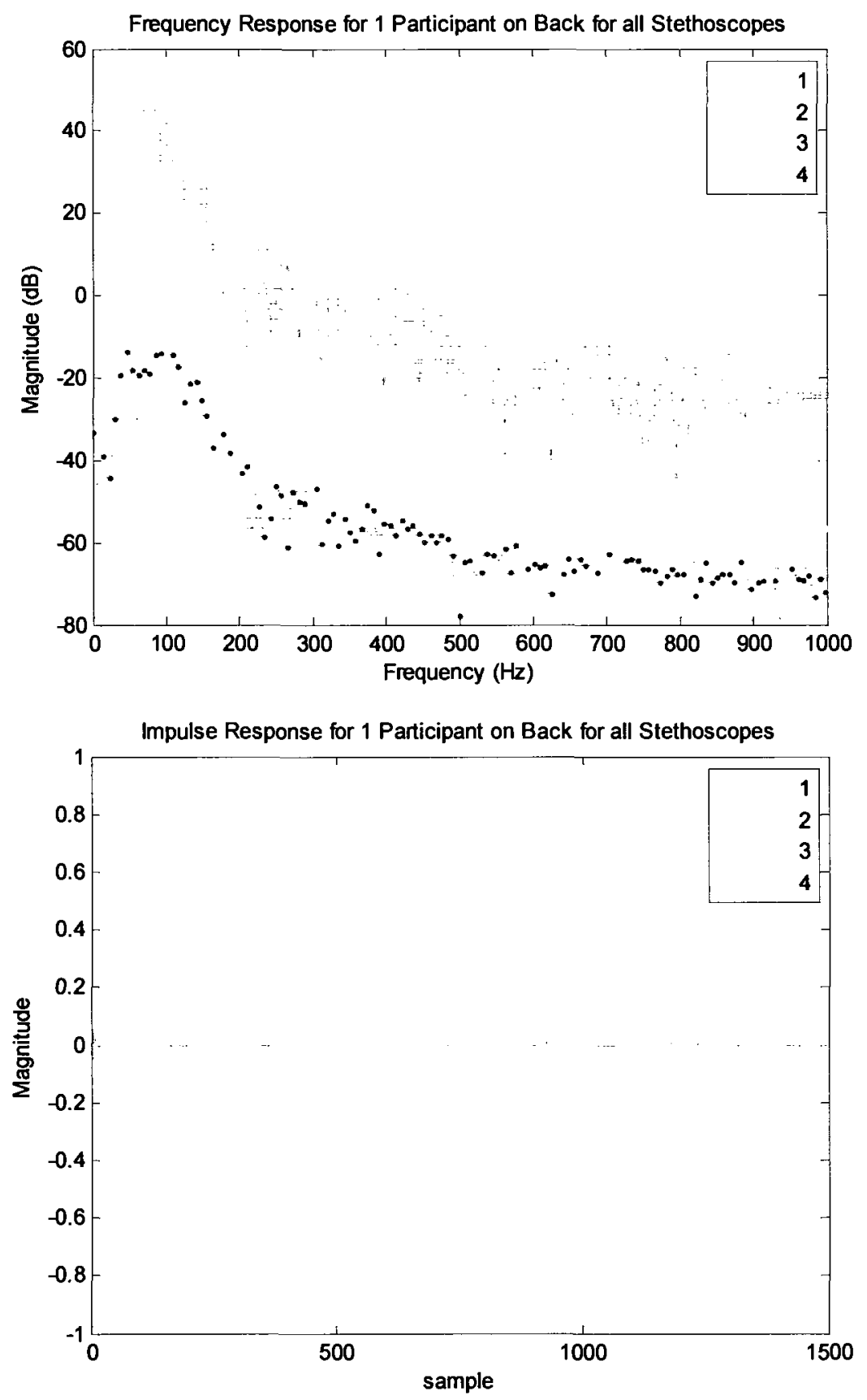

Figure 45 - Frequency and Impulse Response for Participant 3 Lying on Back for all Stethoscopes. The Curve Labelled 1 is the Response on the Stethoscope that was Expected to Yield the Lowest Propagation Delay. 

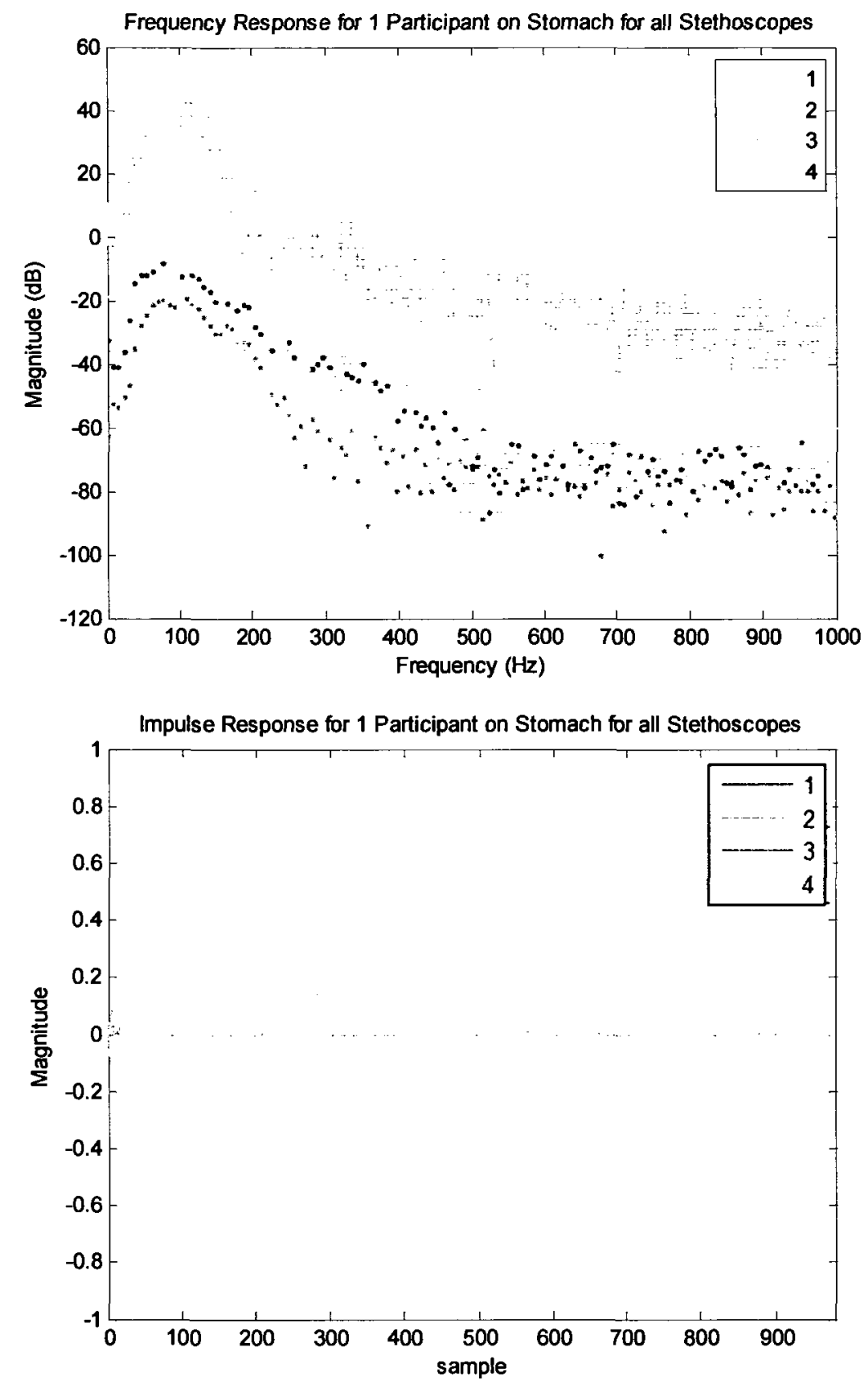

Figure 46 - Frequency and Impulse Responses for Participant 3 Lying on Stomach for all Stethoscopes. The Curve Labelled 1 is the Response on the Stethoscope that was Expected to Yield the Lowest Propagation Delay. 

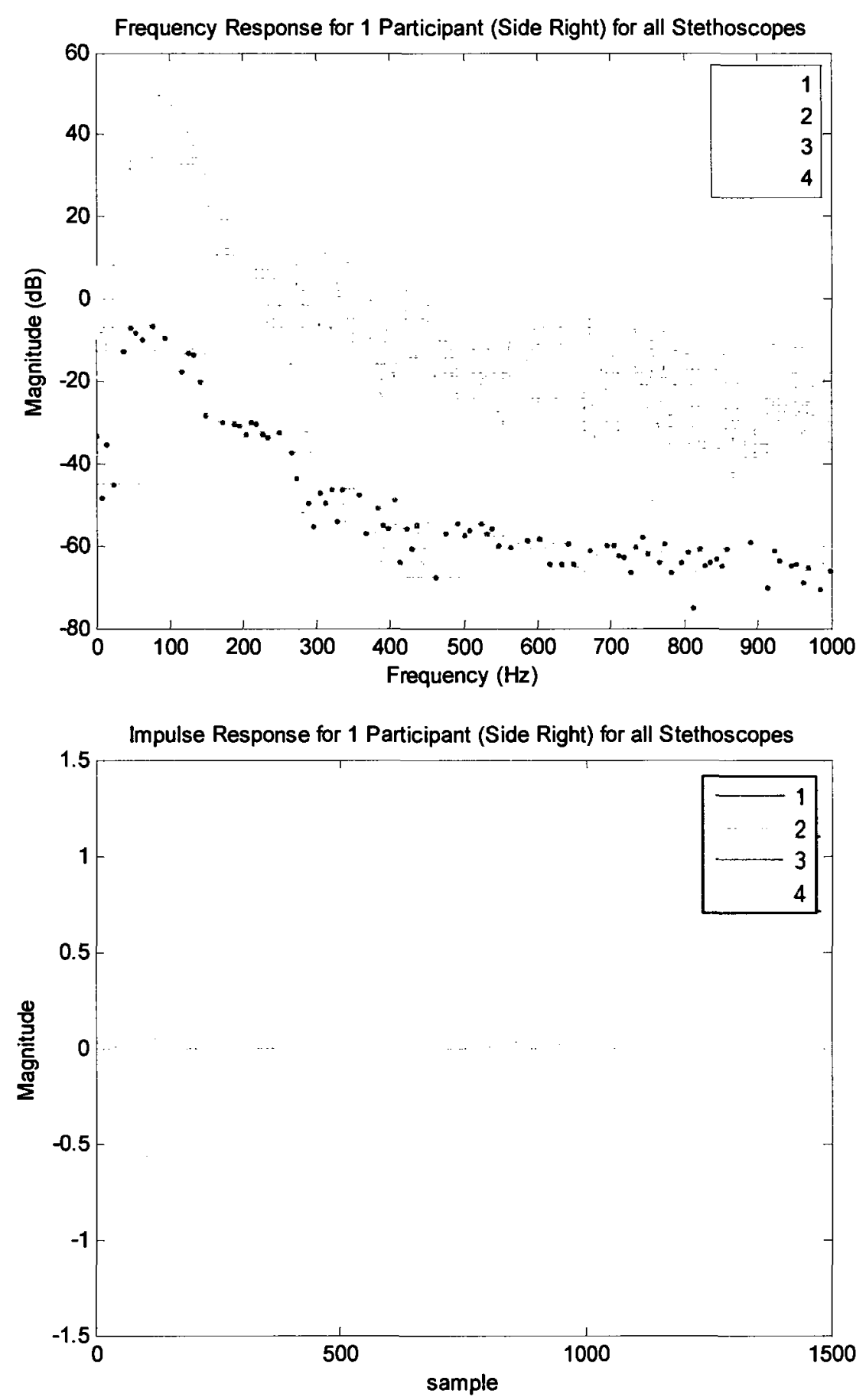

Figure 47 - Frequency and Impulse Responses for Participant 3 Lying on the Right Side for all Stethoscopes. The Curve Labelled 1 is the Response on the Stethoscope that was Expected to Yield the Lowest Propagation Delay. 

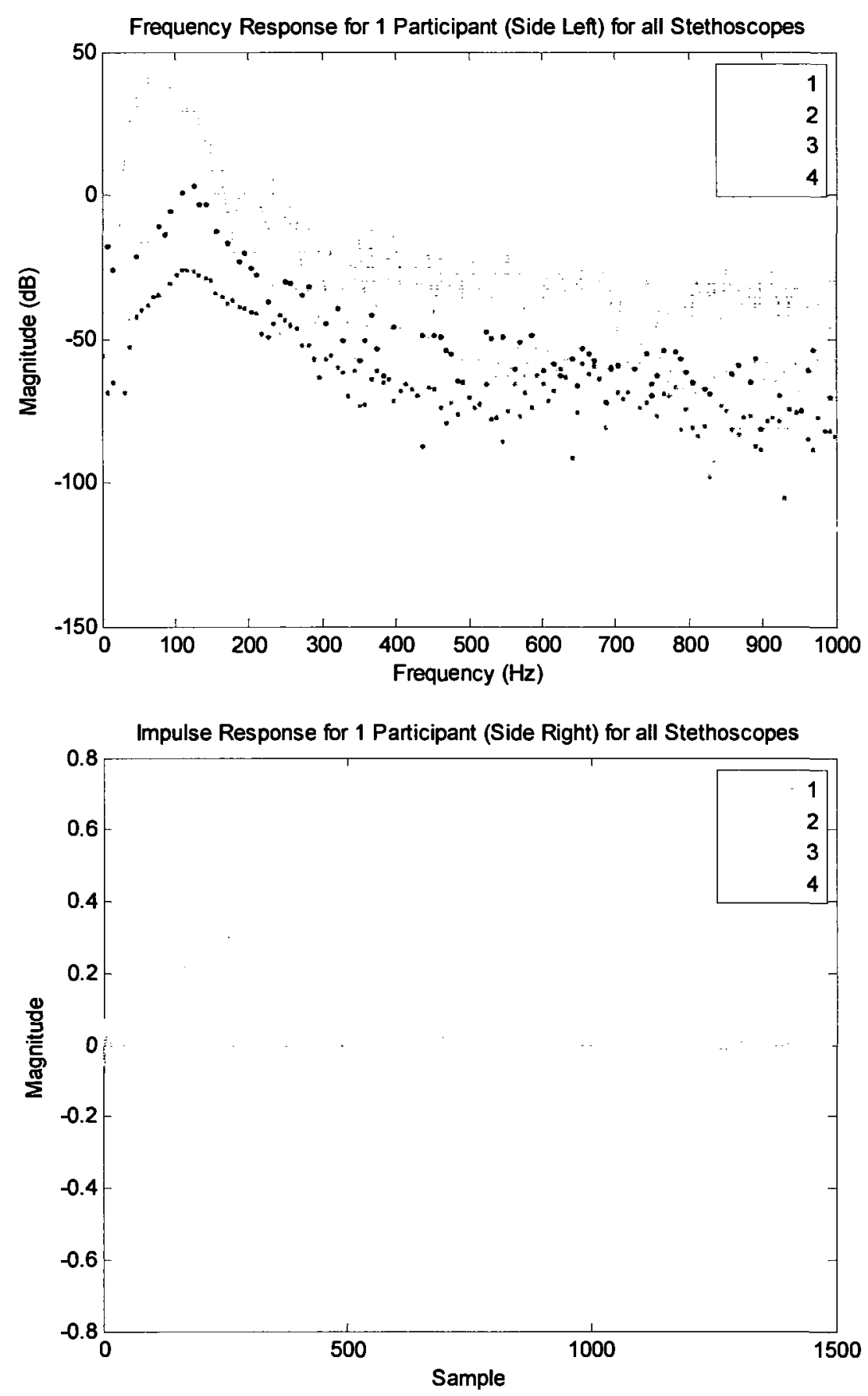

Figure 48 - Frequency and Impulse Responses for Participant 3 Lying on the Left Side for all Stethoscopes. The Curve Labelled 1 is the Response on the Stethoscope that was Expected to Yield the Lowest Propagation Delay. 


\subsection{Chapter Summary}

This chapter discussed experiments performed in vivo. The results were obtained using a specific experimental protocol and showed correlation with in vitro experiments. It was also shown that the medical instrument is capable of detecting variations in the input signals sound propagation delay as a patients posture changes. This was an indication of good resolution capabilities of the instrument. 


\section{Chapter 7 - Non-Linearities within the System}

\section{1 - Non-Linear Systems}

A non-linear system is that in which its output is not linearly proportional to the input into the system and thus cannot be described by a simple linear equation. Adaptive filtering is a method of describing an unknown system using linear operators. When the adaptive filtering algorithm iterates until a minimized error between the systems input and output is achieved, an FIR filter is constructed with a set number of coefficients and their respective magnitudes. The FIR filter is a transfer function capable of describing the systems behaviour with any input signal with any magnitude and phase.

In reality, systems have behaviours that exist that cannot be described solely using linear equations. In adaptive filtering, non-linear signals affect the minimization of the error curve that is important for deciding when to stop iterating through the filtering algorithm. Therefore, if many non-linear components exist within the system, the resulting FIR estimation of the adaptive filtering algorithm will lose accuracy between subsequent runs and varying input signals. The performance of the algorithm depends on what excites the non-linear components of the system (Al-Naffouri \& Sayed, 2001).

\section{2 - Non-Linearities within the System Apparatus}

In this chapter, we discuss non-linearities within the lung monitoring medical instrument. One of the most obvious components that was investigated as a cause for non-linear 
distortions was the loudspeaker. Due to the mechanical nature of a speaker, "harmonic distortions" can occur as the volume of the speaker amplifier is increased. This is because the driver (a lightweight diaphragm) moves within the speaker. As the volume increases, the distance that the diaphragm travels increases. When the distance that the diaphragm travels increases, the loudspeaker reaches a non-linear range filled with distorted harmonics. When the diaphragms' maximum limit is reached (i.e. the input signal voltage reached the current swing capacity of the power supply) "clipping" occurs and the output signal is filled with non-linear discontinuities (Harris, 2009). For the lung monitoring system, it was assumed that non-linearities within the chest phantom models and the human chest were small enough to be ignored. Therefore, in order to test our assumption, multiple test conditions for the system transfer function for human and phantom chests must be satisfied: 1) the system transfer function must be equal with a constant input signal magnitude and 2) the system transfer function must be equal if the input signal magnitude was varied (i.e. loudspeaker volume). The need to verify the second condition is intuitive because the system is assumed to be linear. As an example, if the system transfer function halves the input signal and delays it by one sample then the systems impulse response can be described as an FIR filter with all coefficients equal to zero except the $2^{\text {nd }}$ with a value of a half. Therefore, no matter what magnitude above 0 the input signal is, the systems transfer function resulting from the adaptive filtering process should never change.

The $1^{\text {st }}$ condition in testing that non-linearities were minimal in affecting the results of the system was verified by performing experiments on both human and phantom chests over 
195 trials and verifying that the system transfer function obtained by the adaptive filtering algorithm remained constant. The metric that was used to verify similarities between the transfer functions was the location of two frequency peeks that were prominently observed in both the human and phantom chest experimental trials.

The $2^{\text {nd }}$ condition was verified by varying the volume of the loudspeaker and thus changing the magnitude of the input signal on a human participant. For this experiment, the loudspeaker was set to $100 \%$ volume level and then reduced by $20 \%$ each trial until the $20 \%$ volume level was reached. An average impulse response of the system was obtained using the adaptive filtering algorithm over 15 trials at each volume level. The 5 impulse responses were plotted and compared. If non-linearities were negligible, then all 5 impulse responses were expected to overlap completely. This was not the case at all volume levels seen in Figure 49. This result shows that there are relevant non-linearities within the entire system (including the sound emitting and recording apparatus). The next step was to determine if the non-linearities could be associated to the loudspeaker which was an obvious suspect. 


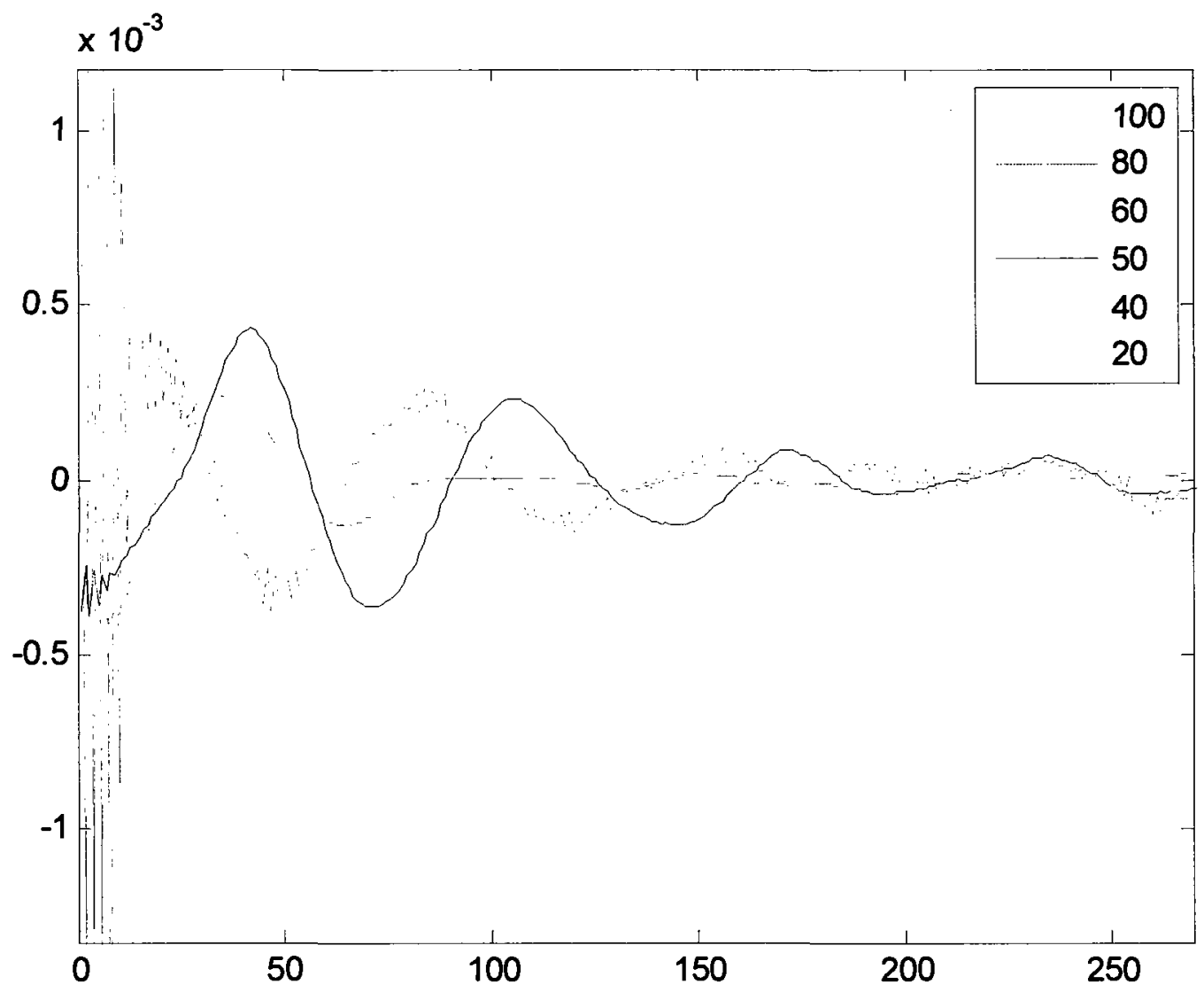

Figure 49 - Impulse Responses of the System at Varying Volume Levels for the Input Signal

In order to determine if non-linearities were being produced by the loudspeaker, the measurement apparatus was re-arranged such that the input signal to the system could be measured before it entered the human or phantom chests. In order to do this, a stethoscope was placed just in front of the loudspeaker without contacting its case in order to avoid possible high frequency resonance from the speaker mesh to the stethoscopes metal housing. Another stethoscope was placed on the surface of a single human chest (this experiment was only performed on a human). The input signal was then played and recorded using the standard measurement apparatus. Figure 50 illustrates a block diagram of the modified lung monitoring system for detection of non-linearities caused by the loudspeaker. The modification was the placement of a stethoscope between 
the speaker and the funnel to capture the input signal as it was emitted from the speaker. The second stethoscope was placed on the chest of the human participant. Both captured signals will include the same non-linear components in their signals and those components will be subtracted in the adaptive filtering process.

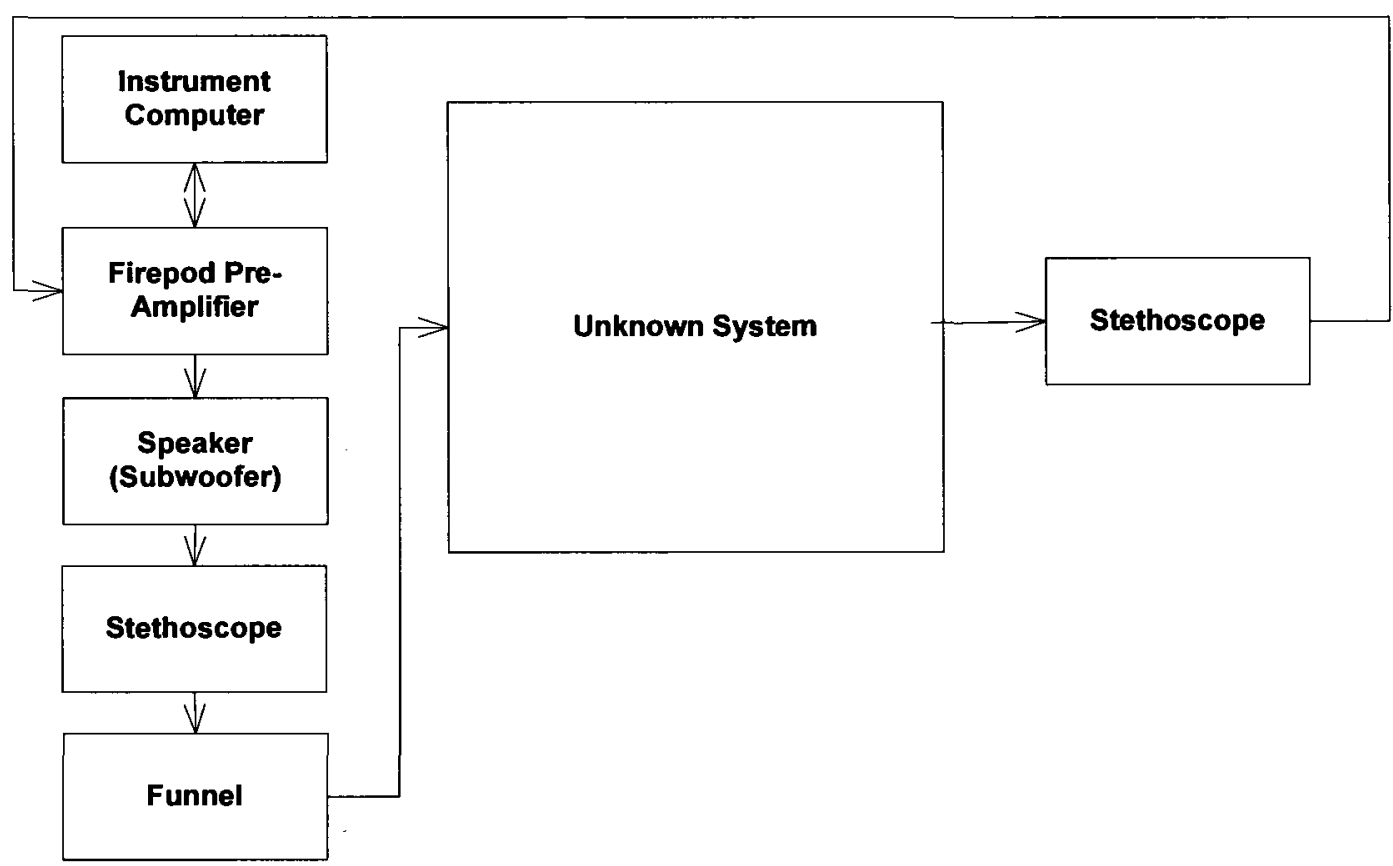

Figure 50 - Block Diagram of the Modified Lung Monitoring System in or to Detect the Source of Non-Linearities

Following the experiment, the impulse response of the signal measured closest to the speaker and the input signal feed directly into the pre-amplifier were obtained for each loudspeaker volume. The impulse responses for loudspeaker volume levels between $20 \%$ and $40 \%$ were very closely matched. Outside of these ranges, the impulse responses did not match as shown in Figure 51 and more clearly in Figure 52 and Figure 53 as normalized versions of Figure 51. 


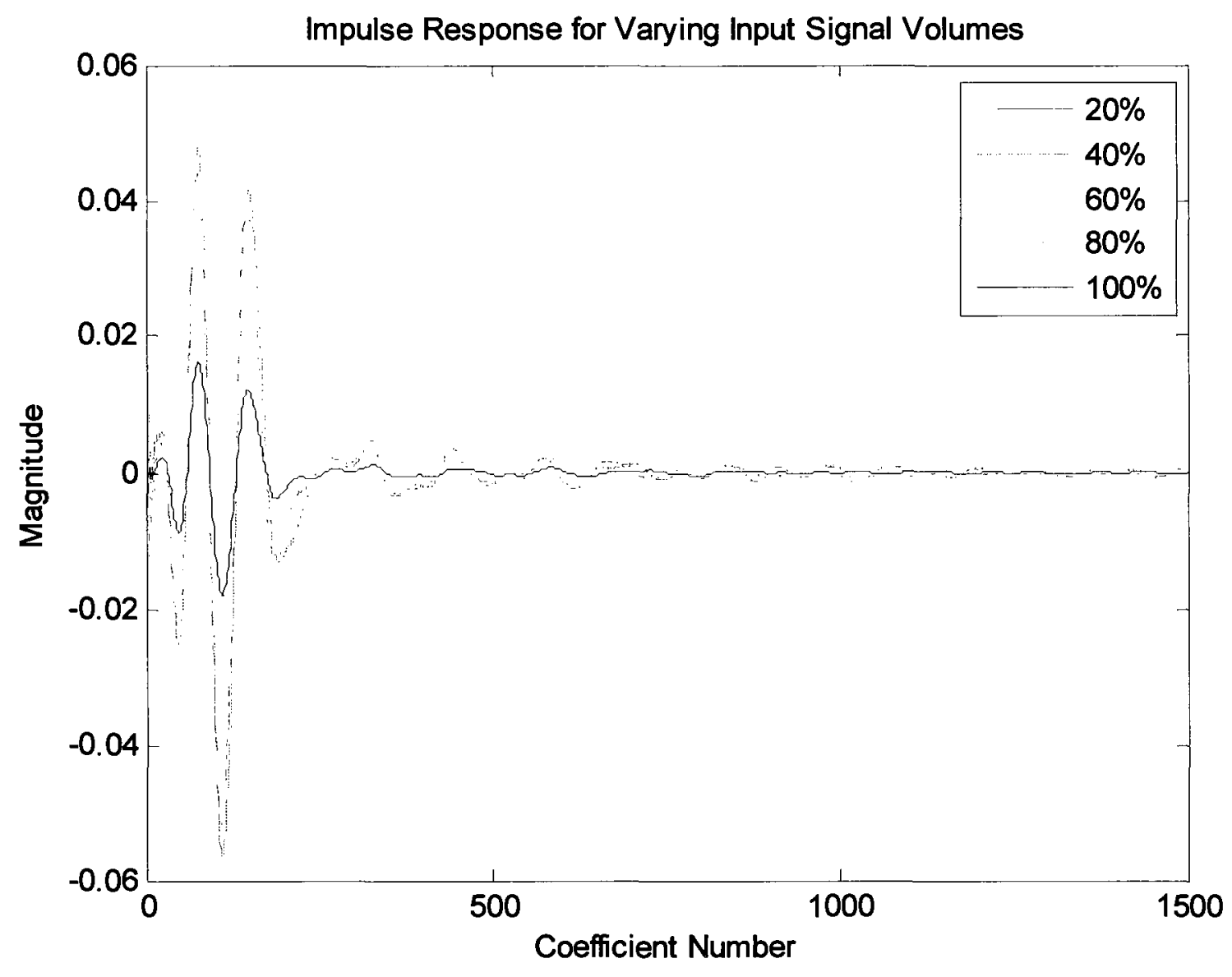

Figure 51 - Impulse Response of the Loudspeaker at Varying Input Signal Volumes 


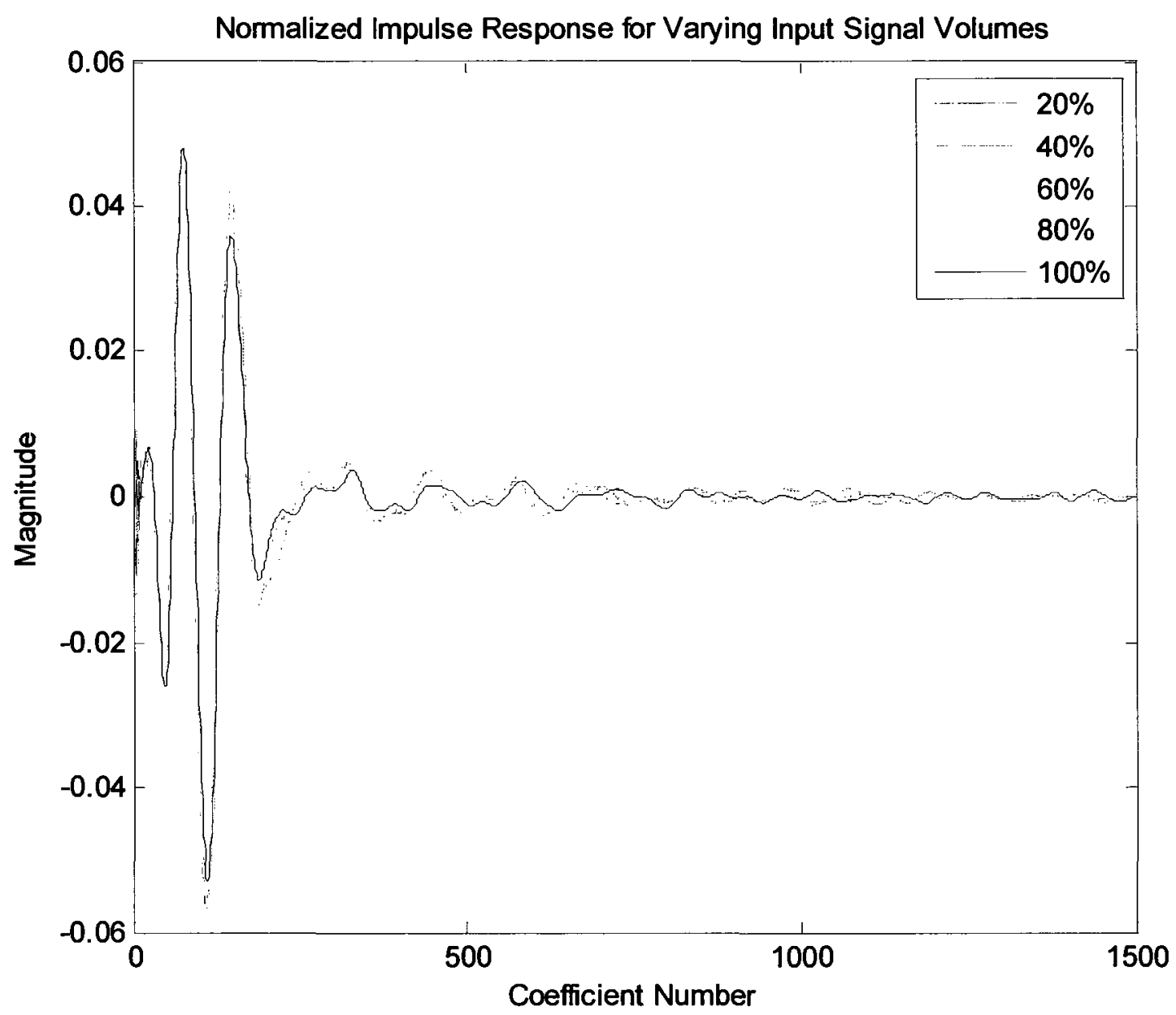

Figure 52 - Normalized Impulse Response of the Loudspeaker at Varying Input Signal Volumes 


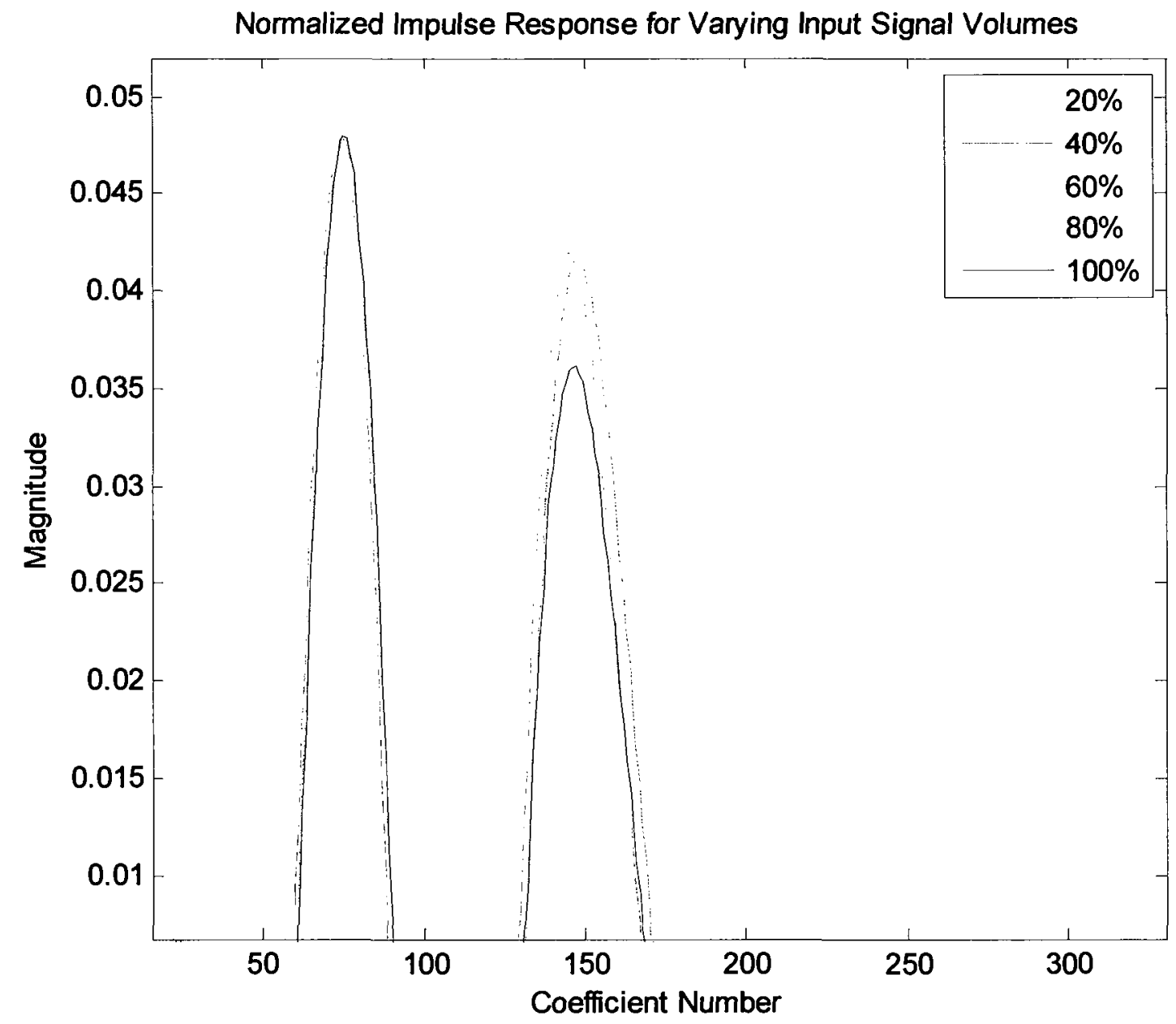

Figure 53 - Zoomed Plot of Normalized Impulse Response of the Loudspeaker at Varying Input Signal Volumes. Second Peak at Volume Levels of $20 \%$ and $40 \%$ Match Closely compared to Other Volume Levels

The next step was to obtain the impulse response at varying volumes between the input signal measured at the speaker and the output signal measured on the surface of the chest. Both signals contain non-linearities from the loudspeaker and these non-linearities will subtract due to the nature of the adaptive filtering algorithm. Therefore, the error signal used to determine the number of iterations for the adaptive filtering process will not be affected by the non-linearities. The system transfer function between the signals should be constant at any volume. 


\section{3 - Chapter Summary}

This chapter demonstrated that non-linearities did have a significant impact on the system. Fortunately these non-linearities were caused by the sound emitting portion of the lung monitoring apparatus and not the human chest. Although certainly nonlinearities do exist within these systems, they were not prominent enough to alter the system transfer functions between subsequent runs. The investigation of non-linear components when building a linear model for an unknown system is critical for any thesis. 


\section{Chapter 8 - Conclusions and Future Work}

This thesis aimed at the development of a computer-aided medical instrument capable of measures changes in the distribution and density of fluid within the lungs caused by diseases including asthma, bronchitis, emphysema, and pneumonia. Possible scenarios where the instrument could be used include: the determination of the effectiveness of various respiratory disease treatments, or the development of lung ventilation strategies to avoid Ventilator Induced Lung Injury (VILI) through the analysis of the relative volume change of mucus in the anterior and posterior chest and abdominal regions following the initiation of a treatment or changes in posture. The medical instrument was also capable of providing spatial information of the chest with respect to the location of possible respiratory obstructions within the pulmonary system.

Benefits to using an array of electronic stethoscopes eliminated possible breath variations between auscultation points as normally physicians measure a series of independent breaths at various locations as opposed to one breath at many locations. This ability provides physicians with a means to more accurately diagnose and monitor respiratory disease. Integrating the stethoscope array with a software tool provides physicians with the ability to playback sounds captured by the stethoscope arrays in a variety of manners.

The investigation of monitoring respiratory diseases began with the development of a series of homogenous phantom models of the human chest for verification of the data 
processing algorithm of the medical instrument and to also attempt to simulate the behaviour of actual human chests. The three models derived in this project were the: open-air column, plastic bucket, and chest phantom models. Upon completion of a reliable computer-aided medical instrument software tool, human trials were conducted and results correlated closely between human and chest phantom models. The instrument was also shown to be able to detect fine changes in sound propagation delay with various in human postures when breathing at tidal volume.

This project showed that adaptive filtering is a promising method of analyzing sound propagation through the human chest to monitor fluid related respiratory disease. The instrument was capable of detecting sound propagation delay changes in a chest phantom model as the volume of water within the model was increased. As expected the sound propagation delay decreased as the volume of water increased within the chest phantom because the speed of sound propagating through water is approximately five times that in air. The delay estimation results for the chest phantom model with no water injected yielded comparable delays to those found for in vivo trials with three healthy male participants. Transfer functions were also compared between the in vivo and in vitro trials. These results suggest the existence of two peak frequencies that sharpened as the volume of water increased within the chest phantom. The sharpening effect was related to a reduction in the sound propagation delay. This was also validated in vivo as changes in posture affect the geometry of the lungs. As the lung compress, the sound propagation path of the input signal through the lungs begins to resemble a cylinder of water due to an increased amount of lung tissue in the sound propagation path. Lung tissue has similar 
properties to water and stethoscopes in the array around the location of the compressed lung detected a reduction in the propagation delay of the input signal compared to other stethoscopes in the array.

Using adaptive filtering to characterize the human chest assumes that there exists a linear transfer function capable of representing the system. In any project where there is an underlying assumption that the system at hand is linear, an investigation into nonlinearities is crucial. In this project the majority of non-linearities existed within the sound emitting component of the medical instrument. This problem was corrected by restricting the sound amplification operating range of the speaker between $20 \%-40 \%$ of its amplification range.

Future work involves the discovery of new parameters that can be derived from adaptive filtering in order to characterize the relative volume of mucus within the respiratory system. Experiments must also be conducted in order to determine how much data is required to achieve the same results as those in this project. A reduction of the input sound signal duration into the mouths of patients would provide them with more comfort. In vivo trials must be conducted on patients with respiratory diseases to see if there is further correlation with the chest phantom model. Finally, a graphical user interface would also need to be developed for the software tool such that physicians can use the tool and be provided with feedback. 


\section{References}

Adler, A. (2008, September). Engineering Analysis and Modelling of Human Anatomy and Physiology: Pulmonary System Lecture 3. Ottawa, Ontario, Canada.

Al-Naffouri, T. Y., \& Sayed, A. H. (2001, January). Adaptive Filters with Error Nonlinearities: Mean-Square Analysis and Optimum Design. EURASIP Journal on Applied Signal Processing, pp. 192-205.

Auscultation. (2008). Retrieved June 14, 2008, from Encyclopedia Britannica On-Line: http://www.britannica.com/eb/article-9011297/auscultation

Bergstresser, T., Ofengeim, D., Vyshedskiy, A., Shane, J., \& Murphy, R. (2002). Sound Transmission in the Lung as a Function of Lung Volume. Journal of Applied Physiology, 667-674.

Bray, D., Reilly, R. B., Haskin, L., \& McCormack, B. (1997). Assessing Motility Through Abdominal Sound Monitoring. IEEE EMBS, pp. 2398-2400.

Canter, L. W., Knox, R. C., \& Fairchild, D. M. (1988). In D. M. Fairchild, Ground Water Quality Protection (pp. 44-45). CRC.

Carlson, N. R., Buskist, W., Enzle, M. E., \& Heth, C. D. (1997). Psychology: The Science of Behaviour. Allyn \& Bacon.

Computed Tomography (CT). (2009). Retrieved September 14, 2009, from The U.S FDA: http://www.fda.gov/Radiation-

EmittingProducts/RadiationEmittingProductsandProcedures/MedicalImaging/MedicalXRays/ucm115317.htm

Definition: Cilia. (2007). Retrieved June 25, 2008, from Asthma Glossary: Cleveland Clinical Health System: http://www.cchs.net/health/healthinfo/docs $/ 2100 / 2186$.asp?index $=8959$

Definition: macrophages. (2005). Retrieved June 25, 2008, from Northwest Biotherapeutics: http://www.nwbio.com/glossary_of_terms.php

Dreyfuss, D., Saumon, G. (1998). Ventilator-Induced Lung Injury: Lessons from Experimental Studies," Respiratory Critical Care Medicine, 294-323

DS32A Digital Electronic Stethoscope. (n.d.). Retrieved June 26, 2008, from ThinkLabs Inc.: http://www.thinklabsmedical.com/electronic-stethoscope/products/ds32a-digitalelectronic-stethoscope-157.html 
Ertel, P. Y., Lawrence, M., Brown, R. K., \& Stern, A. M. (1966). Stethoscope Acoustics II: Transmission and Filtration Patterns. Journal of the American Heart Association, 899-909.

Fishleder, A. J., \& Rothner, A. D. (2008, 08 15). Pulmonary Rehabilitation: Is it for you? Retrieved 07 26, 2009, from The Cleveland Clinic:

http://my.clevelandclinic.org/disorders/Chronic_Obstructive_Pulmonary_Disease_copd/h ic_Pulmonary_Rehabilitation_Is_it_for_You.aspx

Harris, T. (2009). How Speakers Work. Retrieved May 15, 2009, from How Stuff Works: http://electronics.howstuffworks.com/speaker.htm

Haykin, S. (2002). Adaptive Filter Theory. Prentice Hall.

Illustration of Human Respiratory System. (2008). Retrieved June 25, 2008, from The New York Times Company: http://seniorhealth.about.com/library/conditions/blresp.htm

Kaelin, M. (2001). Professionalization of Exercise Physiology. Journal for Exercise Physiologists .

Kompis, M., Pasterkamp, H., \& Wodicka, G. R. (2001). Acoustic Imaging of the Human Chest. Chest Journal , 1309-1321.

Kraman, S. S. (1980, August). Determination of the Site of Production of Respiratory Sounds by Subtraction Phonopneumography. The American Review of Respiratory Disease , pp. 303-309.

Leuppi, J., Dieterle, T., Wildeisen, I., Martina, B., Tamm, M., Koch, G., et al. (2005). Can Airway Obstruction be Estimated by Lung Auscultation in an Emergency Room Setting? Respiratory Medicine, pp. 279-285.

Lin, B. S., Wu, H. D., Chong, F. C., \& Chen, S. J. (2006, February). Wheeze Recognition Based on 2D Bilateral Filtering of Spectrogram. Biomedical Engineering Applications Basis Communications , pp. 128-137.

Lundqvist, H. (2008). Ultrasound in Diagnostics and Therapy. Uppsala, Sweden: University of Uppsala.

Lung Sound Auscultation Trainer. (2008). Retrieved June 26, 2008, from Kyoto Kagaku Co.: http://www.kyotokagaku.com/products/detail01/pdf/m81-s_catalog.pdf

Maddox, L., \& Schwartz, D. A. (2002, February). The Pathology of Asthma. Annual Review of Medicine , pp. 477-498.

Marieb, E. N. (1995). Human Anatomy and Physiology 3rd. Edition. The Benjamin/Cummings Publishing Company, Inc. 
Martinez-Alajarin, J., Lopez-Candel, J., \& Ruiz-Merino, R. (2007). Classification and Diagnosis of Heart Sounds and Murmurs Using Artificial Neural Networks. Springer Link, pp. 303-312.

McKee, A. (2004). Beamforming for multisensory Stethescopes. Carleton university.

Mulligan, K., Adler, A., \& Goubran, R. (2009). Detecting Regional Lung Properties using the Audio Transfer Function of the Respiratory System. International Conference of the IEEE Engineering in Medicine and Biology Society. Minneapolis, MN.

Mulligan, K., Adler, A., \& Goubran, R. (2009). Monitoring Lung Disease using Electronic Stethoscope Arrays. Canadian Medical and Biological Engineering Society. Calgary, AB.

Paciej, R., Vyshedskiy, A., Shane, J., \& Murphy, R. (2002). Transpulmonary Speed of Sound Input into Supraclavicular Space. Journal of Applied Physiology, 604-611.

Pneumonia. (n.d.). Retrieved June 20, 2008, from Free Health Encyclopedia.

Rajanala, S., Jean, S., Kushnir, I., Dellinger, R. P., \& Parrillo, J. E. (2005). Vibration Response Imaging in Critically Ill Patients in the Intensive Care Unit. Chest Journal, 409-495.

Rice, D. A. (1982). Sound Speed in Pulmonary Parenchyma. Journal of Applied Physiology, 304-308.

Schriber, A. (2007). Breath Sounds. Retrieved June 26, 2008, from Medline Plus: Medical Encyclopedia: http://www.nlm.nih.gov/medlineplus/ency/article/003323.htm

Solomon, E., Berg, L. R., \& Martin, D. W. (1999). Biology 5th Edition. Saunders College Publishing/Harcourt Brace College Publishers.

Webster, J. G. (1998). Medical Instrumentation: Application and Design 3rd Edition. New York: John Wiley \& Sons. 